\title{
Hadronic Particle Production in Nucleus-Nucleus Collisions
}

\author{
P. Senger ${ }^{1}$ and H. Ströbele ${ }^{2}$ \\ ${ }^{1}$ Gesellschaft für Schwerionenforschung, Darmstadt \\ ${ }^{2}$ Fachbereich Physik \\ Johann Wolfgang Goethe-Universität Frankfurt/Main
}

\begin{abstract}
Data on hadronic particle production in symmetric nuclear collisions from SIS/BEVALAC to SPS energies are reviewed. The main emphasis is put on the production of pions, kaons, and antibaryons. Global features will be discussed in terms of rapidity and transverse momentum distributions and the total energy stored in produced particles. Pion and kaon production probabilities are studied as function of beam energy and their distribution in polar and azimuthal angle. Special emphasis is put on medium effects expected for kaons in dense nuclear matter at low energies. An enhanced strange particle yield is found at all energies, its explanation at SPS energies is still controversial. Experimental data on antibaryon and multistrange hyperon production is less complete and does not allow for similar systematic studies.
\end{abstract}




\section{Contents}

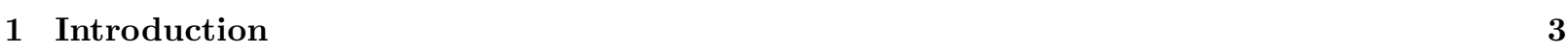

\begin{tabular}{|ll|l}
2 & Global features & 5
\end{tabular}

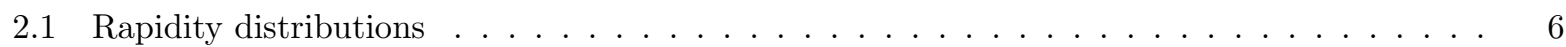

2.2 Transverse momentum distributions $\ldots \ldots \ldots \ldots \ldots \ldots \ldots$

$2.3 \quad$ Energy in produced particles $\ldots \ldots \ldots \ldots \ldots \ldots \ldots$

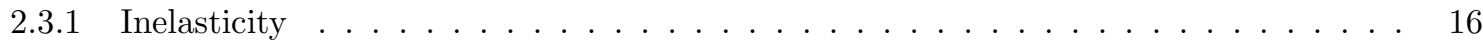

$2.3 .2 \quad$ Fraction of energy in strange particles $\ldots \ldots \ldots \ldots \ldots \ldots$

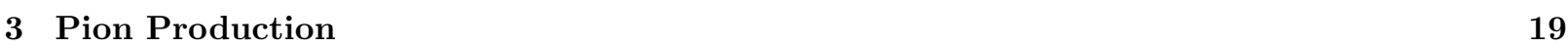

$3.1 \quad$ Multiplicities . . . . . . . . . . . . . . . . . . . . . . . . . . . . . . . 19

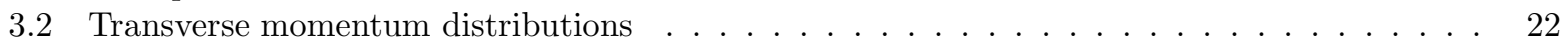

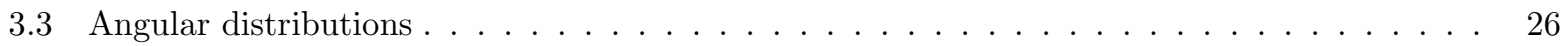

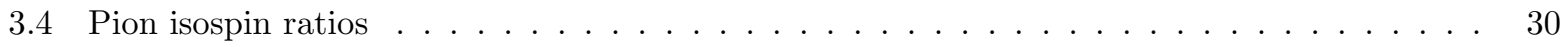

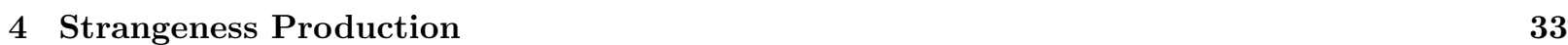

4.1 Kaon production probabilities $\ldots \ldots \ldots \ldots \ldots \ldots \ldots \ldots$

4.2 Kaon spectral distributions $\ldots \ldots \ldots \ldots \ldots$

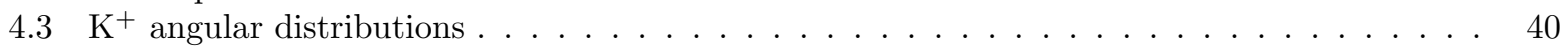

4.4 Probing the nuclear equation of state $\ldots \ldots \ldots \ldots \ldots \ldots$. . . . . . . . . . . . 42

4.5 Kaons and antikaons in dense nuclear matter $\ldots \ldots \ldots \ldots \ldots$. . . . . . . . . . 45

4.6 Strange meson production at ultrarelativistic energies $\ldots \ldots \ldots \ldots \ldots$

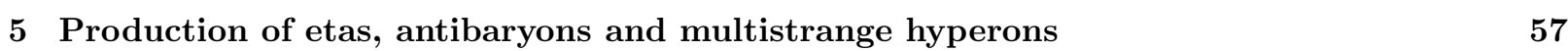

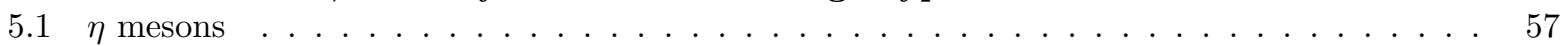

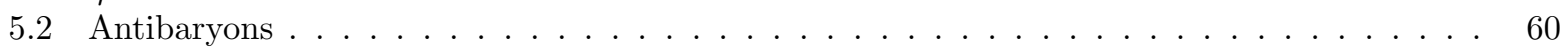

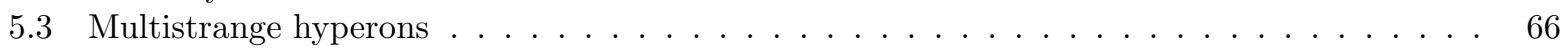

\begin{tabular}{|lr}
\hline 6 Conclusions & 68 \\
\hline
\end{tabular} 


\section{Chapter 1}

\section{Introduction}

Relativistic nucleus-nucleus collisions offer the unique possibiliy to produce hadronic matter at high temperatures and densities in the laboratory. At center-of-mass energies in the range from 0.2 to $10 \mathrm{GeV}$ per nucleon, temperatures of 50 to about $200 \mathrm{MeV}$ and baryonic densities up to 10 times normal nuclear matter density are reached. The variation of density with energy allows to extract information about the nuclear equation of state which is essential for the understanding of the dynamics of supernovae and the formation of neutron stars. The hadrons inside the dense baryonic medium are expected to change their free particle properties due to the onset of chiral symmetry restoration. At energy densities above a few $\mathrm{GeV} / \mathrm{fm}^{3}$ the hadrons in the reaction zone can no longer exist as discrete entities; they probably dissolve into a plasma of quarks and gluons. Such a state of matter is predicted to prevail in the interior of neutron stars and believed to have existed in the early universe. Hence, heavy ion reactions at relativistic energies are an essential tool for the investigation of cardinal questions in nuclear and subnuclear physics.

Nuclear collisions are complex multibody reactions in which three stages may be identified:(1) the interpenetration of the nuclei with highly non equilibrium hadronic - and at high energies partonic interactions; (2) the 'burning' of the fireball with its evolution towards chemical and thermal equilibrium; (3) the 'freeze-out' of the final state hadrons. The crucial questions concern the second and third stage: does the system "live" long enough to behave as bulk matter and do the produced particles contain information about the matter they originate from? The experimental data on the ratios of produced particles and their distributions in phase space indeed indicate that they are emitted from an expanding source which is close to thermal and chemical equilibrium. Once the steady state features of nuclear collisions are established, fundamental aspects of strong interactions come into focus: the modification of hadron properties in the hadronic medium and the deconfinement of the quarks with its implications for the understanding of the physical vacuum.

There are various experimental observables which give access to the physics of a nuclear collision. The multiplicities and momenta of emitted nucleons and of nuclear fragments prevail at low energies. Electromagnetic radiation and lepton pairs are especially interesting because they are penetrating probes which, however, are strongly suppressed relative to hadronic signals. Newly produced hadrons, which are the subject of this article, dominate the final state at high energies. Here they are the main carriers of energy and entropy. Near production threshold, i.e. at low energy, they are interesting observables because their emission characteristics are strongly affected by the interplay between medium and threshold effects. In the following we review hadronic particle production in nucleus-nucleus collisions at c.m. energies in the range from a few hundred $\mathrm{MeV}$ to $10 \mathrm{GeV}$ per nucleon.

The formation of a new hadron implies the creation of at least one $q \bar{q}$ pair and often the rearrangement with the primordial quarks in the initial nucleons. This complex process may be modified in A+A collisions by medium effects like mean field potentials, Fermi motion, Pauli blocking of certain final states normally attained in N-N collisions, and more subtle microscopic effects like reabsorption or the scattering between hadronic resonances. Interactions involving resonances can change considerably the effective accessible phase space and, thus, particle production thresholds in nuclear collisions. A detailed picture of the large 
hadronic system formed in central nucleus-nucleus collision at relativistic energies has to account for the large differences in densities of the different quark flavours. For not too high energies there will always be a high population of the light $u$ and $d$ quarks which are brought in by the projectile nucleons. Thus the reabsorption of the antiquark of a newly created light $q \bar{q}$ pair is a frequent process. If, however, a heavy quark pair like $s \bar{s}$ or $c \bar{c}$ etc. is produced, its annihilation is strongly supressed as long as the density of the corresponding quark species is low. This is equally true for mesons containing a heavy antiquark, for example the $\mathrm{K}^{+}$meson. Thus $\mathrm{K}^{+}$mesons will leave the reaction zone unaffected by reabsorption. The approach to chemical equilibrium of the light quarks will be especially boosted and thus be much faster than for the heavy quarks not only because of the smaller mass but also because of the favourable environment rich in $u$ and $d$ quarks. The result of the equilibration for the different quark flavours and also for the hadron abundances can be worked out in a bulk matter scenario (using the laws of thermodynamics or hydrodynamics). Microscopic transport models can in addition analyse the approach to equilibrium. Both lead to predictions of particle ratios in the final state, which are observable in experiments.

This list of important and interesting subjects pertinent to nuclear collisions demonstrates not only the scientific potential of the field but also its problems: the superposition, attenuation and amplification of many different phenomena will make it difficult to extract parameters of individual elementary processes.

Particle production in nuclear collisions has been a subject of scientific research already for two decades [1]. Relatively light projectiles like $\mathrm{He}, \mathrm{C}, \mathrm{Ne}$, up to Ar had been available at the BEVALAC in Berkeley, at the Synchrophasotron in Dubna, and at the ISR in Geneva (He and D only). The main topics of research were collective effects and the search for exotic phenomena. The latter was unsuccessful whereas the former opened up an avenue to study the bulk properties of nuclear matter far from stability. In particular predictions [2] were confirmed that nuclear matter will exhibit hydrodynamic behavior if compressed to several times nuclear ground state density and if heated to temperatures in excess of a few tens of $\mathrm{MeV}$, 3 , 4, 5, 6. Particle production experiments had their share in the research programme, but the main emphasis was put on the emission characteristics of the nucleons and the nuclear fragments [7]. At ultrarelativistic energies, however, particle production was found to be the dominant feature of nucleus-nucleus collisions [8].

Recently even the heaviest stable nuclei have been accelerated at the BEVALAC, at the SIS of GSI in Darmstadt, at the AGS in Brookhaven, and at the SPS of CERN in Geneva. New high sensitivity experiments at all laboratories and the high energy beams available at CERN lead to new theoretical and experimental efforts to understand particle production in nuclear collisions. Two extreme configurations will be of special importance: At low energies particle production occurs near to the threshold and is thus especially sensitive to collective effects. Low energy in this context means that the c.m. kinetic energy per nucleon of the projectile is of the same order of magnitude as the mass of the produced particle. At high energy densities, parton effects should come into play. In principle this is true also for high energy nucleonnucleon interactions, however, in nuclear collisions the origin of partonic effects is not a large momentum transfer, but rather the overlap of the hadronic particles. Therefore, subnucleonic effects in relativistic heavy ion collisions will set in at lower c.m. energies per nucleon than in the interactions of two nucleons.

In this article, beam energies of a few hundred to a few hundred thousand $\mathrm{MeV}$ per nucleon are considered. This range translates into c.m. kinetic energies (per nucleon) from approximately $200 \mathrm{MeV}$ (BEVALAC/SIS) to about $10 \mathrm{GeV}$ (CERN-SPS). We concentrate on hadronic particle production in highenergy nucleus-nucleus collisions; often we will compare its characteristic features to the ones of nucleonnucleon $(\mathrm{N}-\mathrm{N})$ interactions. The main emphasis will be put on the production of pions, kaons and antibaryons. Other particles like heavy non-strange mesons as well as nucleons will be mentioned only if necessary to round up the picture. Hydrodynamic-like phenomena resulting from pressure, expansion, and directed flow prevail at low energies and are seen almost exclusively in the nucleons, light fragments and their correlations. For a recent review of this subject see [6]. Here we will cover only related phenomena observed in the meson spectra at low beam energies. Although there is a large body of data on asymmetric colliding systems like light projectile on heavy target, we concentrate on symmetric collision systems. 


\section{Chapter 2}

\section{Global features}

In this chapter we will consider the features of nucleus-nucleus collisions in general and distinguish those which are unique to nuclear reactions from others which are also observable in hadronic (i.e. nucleonnucleon) interactions.

A simple, although important feature of nuclear collisions is the fact that the direction and magnitude of the impact parameter can be measured for each individual collision event. The experimental observable for the centrality of the collision is usually the number of nucleons which participate in the collision $\left(\mathrm{A}_{\text {part }}\right)$. Independent of the size of the collision system, $\mathrm{A}_{\text {part }}$ determines the reaction volume and the total centerof-mass energy. For impact parameter integrated (inclusive) cross sections and symmetric collision systems, the mean number of interacting nucleons can be estimated to $\left\langle\mathrm{A}_{\text {part }}>\approx \mathrm{A} / 2\right.$ with $\mathrm{A}$ the mass number of the colliding nuclei [9, 10]. The mean multiplicity of a particle species is given by the ratio of the inclusive production cross section to the reaction cross section $\left.(<M\rangle=\sigma_{i n c l} / \sigma_{R}\right)$ or determined directly from an event-by-event measurement. For comparisons of different size systems it is convenient to use the normalized mean multiplicity $\left\langle M>/ A_{\text {part }}\right.$ (see section 3.1). The direction of the impact parameter defines the reaction plane and can be determined by the sum of the transverse momentum vectors of the projectile spectator fragments 11 .

The most important features of nuclear collisions are collective effects. They are related to the dynamics and the bulk properties of the reaction zone like nuclear stopping, the built-up of pressure, the energy density, thermal and chemical equilibration, the decompressional flow. Some aspects of collective phenomena can be studied by means of global observables like the phase space distributions of the produced particles, the inelasticity of the collisions, the ratios of different particle species and particle correlations. The latter are used to obtain information about the space-time configuration of the reaction zone and are not a subject of of this review (see e.g.12]). The phase space distributions carry information about the degree of thermalisation and collective motion and will be considered in the following section.

Particle ratios can be employed to evaluate the degree of chemical equilibration observed in the final state of hadronic collisions. For nuclear matter in equilibrium, the particle composition is characterized by a temperature $\mathrm{T}$ and various chemical potentials. The particle number densities can be calculated considering a grand-canonical ensemble (as the particle number is not conserved) of noninteracting fermions and bosons:

$$
\rho_{i}=\frac{g_{i}}{2 \pi^{2}} \int_{0}^{\infty} \frac{p^{2} d p}{\exp \left[\left(E_{i}-\mu_{B} B_{i}-\mu_{S} S_{i}\right) / T\right] \pm 1}
$$

where $g_{i}$ is the spin-isospin degeneracy, $E_{i}$ the total energy in the local restframe, $B_{i}\left(S_{i}\right)$ the baryon (strangeness) number and $\mu_{B}\left(\mu_{S}\right)$ the baryon (strange) chemical potential. In general one has to modify expression 2.1 in order account for the finite size of the system, for the volume of the particles ("surface" and "excluded volume" corrections, see [14) and for resonance decays which occur in a later stage of the collision. The results of such an analysis for particle production data from nuclear collisions at SIS [13], AGS [14, 15], and SPS [15, 16] energies show consistently that the relative abundances of different particle 


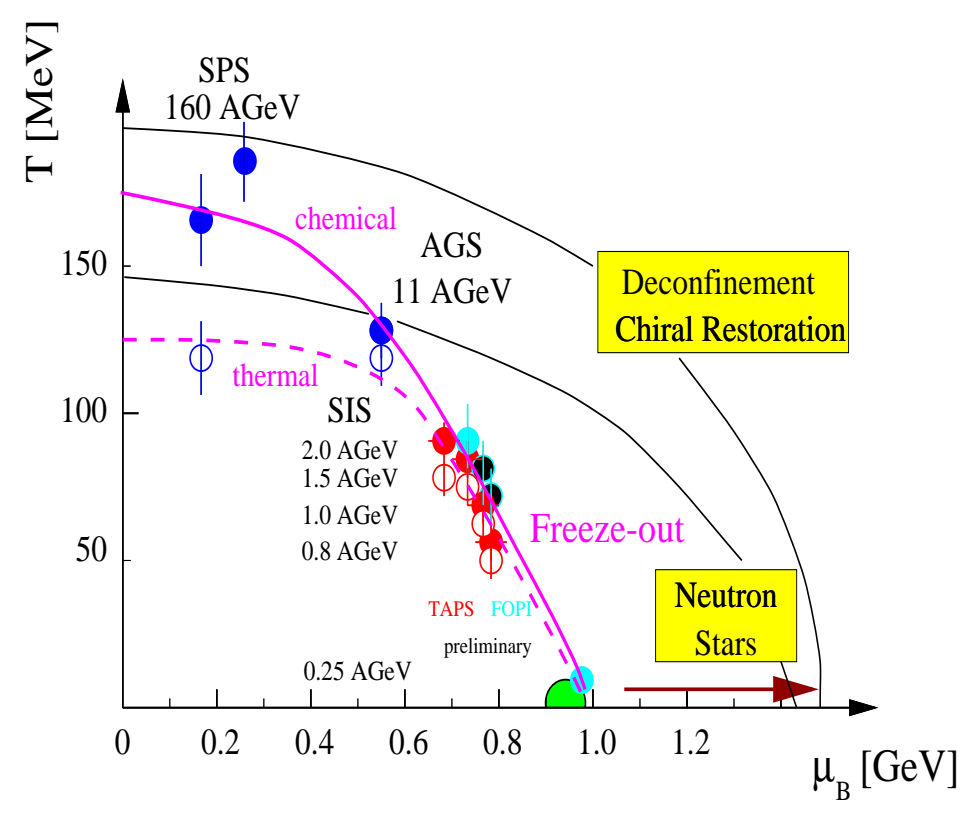

Figure 2.1: Phase diagram of hadronic matter: the temperature $\mathrm{T}$ and the baryochemical potentials $\mu_{B}$ are derived from yield ratios of particles produced in nucleus-nucleus collisions at different incident energies. The thick solid line through the data points represents the curve for chemical freeze-out of hadronic matter. Open data points (connected by the dashed line) represent parameter pairs for thermal freezeout. The figure is taken from 113, the results are from $13,14,16,17,18$.

species are close to what is expected for a system in chemical equilibrium. Fig. 2.1 presents the resulting phase diagram of hadronic matter which suggests that for SPS and AGS energies the collision system at freeze-out is close to the phase transition to the quark-gluon-plasma.

Moreover it has recently been shown that hadronic final states in high energy $\mathrm{e}^{+}+\mathrm{e}^{-}$and $\mathrm{p}+\mathrm{p}$ interactions also fulfil the criteria of chemical equilibrium [19] if a suppression of strange particles is assumed. This behavior is expected if particle production is a microscopic process involving many particles and interactions. The appearance of a new hadron is always preceeded by the creation of a quark-antiquark pair. In the next step one of the quarks is normally exchanged with another one from the surrounding medium (hadron or string). Finally the quarks get dressed to become constituent quarks. It is conceivable that this complicated multistep and multibody process probes the available phase space which will lead to a thermodynamic behavior of the produced particles.

The finding of thermodynamic behavior in both nucleus-nucleus and nucleon-nucleon collisions can be interpreted in the following way: All hadrons are created by processes involving the partonic medium which constitutes the interior of hadrons. In hadronic interactions strong constraints on energy, momentum, and quantum numbers affect the configuration of the final state hadrons. In central $\mathrm{A}+\mathrm{A}$ collisions the constraints are losened and a grand canonical ensemble emerges. Therefore, the resulting hadronic fireball is close to thermal and chemical equilibrium because the prehadronic partonic processes randomly filled phase space according to the laws of statistics rather than because of many elastic and inelastic collisions.

\subsection{Rapidity distributions}

Particle spectra are often treated separately in longitudinal and transverse directions. This is because longitudinal velocities are mostly relativistic. The corresponding momenta need therefore a relativistic treatment which is handled most easily when using the rapidity

$$
y=0.5 \cdot \ln \left\{\left(E+p_{L}\right) /\left(E-p_{L}\right)\right\}
$$

with $E$ and $p_{L}$ being the energy and longitudinal momentum of the particle. Such a representation guarantees that the shape of the corresponding distribution is independent of the Lorentz frame 1 .

Rapidity and transverse momentum distributions allow to address some important questions on the reaction dynamics and on properties of the particle emitting source: Are the nucleons slowed down to the

\footnotetext{
${ }^{1}$ On the other hand it implies that a possible kinematical correlation of the considered particles with the projectiles in their c.m.-frame may be hidden. In such a case the Feynman $\mathrm{x}$ variable $\mathrm{x}_{F}=\mathrm{p}_{L} / \mathrm{p}_{\max }$ is more appropriate
} 
extend that their motion is entirely thermal or is nuclear matter becoming transparent at high energies, is the source spherical symmetric or elongated, is there a transverse and longitudinal expansion, is the source thermally equilibrated?
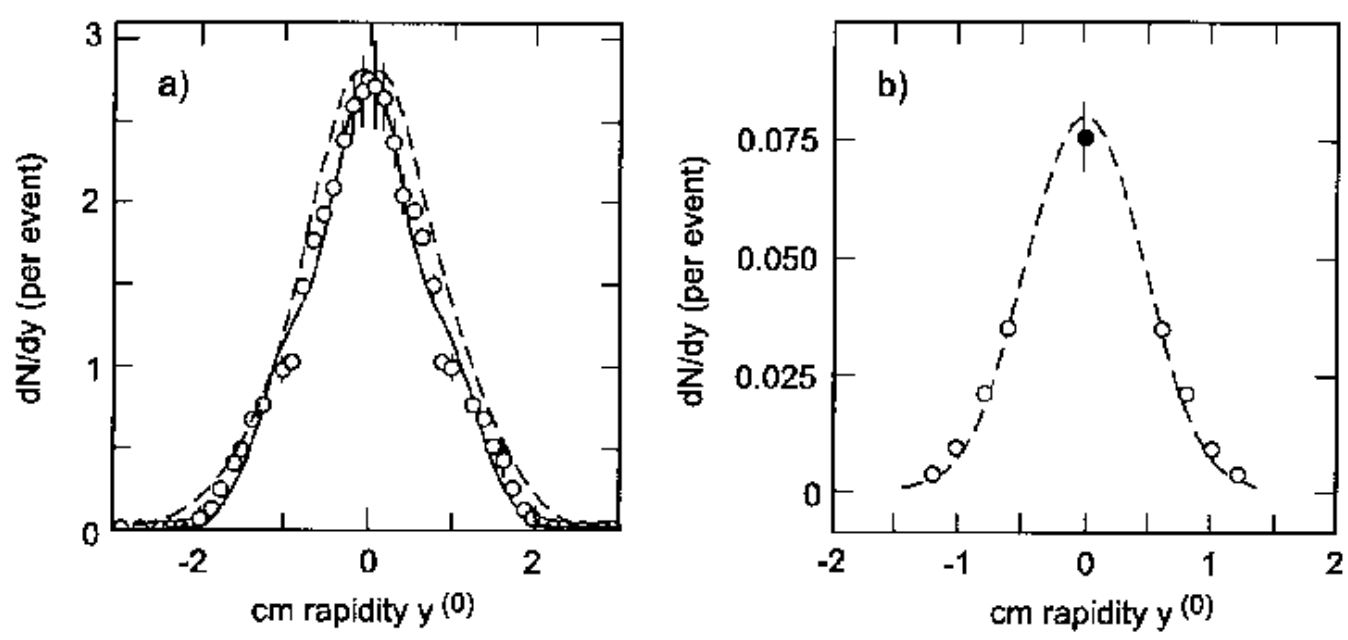

Figure 2.2: Rapidity density distributions of $\pi^{-}$mesons from central $\mathrm{Au}+\mathrm{Au}$ collisions at $1.06 \mathrm{AGeV}$ (left) 222 and of $\mathrm{K}^{+}$mesons from $\mathrm{Ni}+\mathrm{Ni}$ collisions at $1.93 \mathrm{AGeV}$ (right) 21]. The data are measured in the backward hemisphere $\left(\theta_{\text {lab }}^{\pi}>30^{0}, \theta_{\text {lab }}^{K^{+}}>44^{0}\right)$ and symmetrized with respect to midrapidity. The solid line (left) is a fit to the data assuming three thermal pion sources 22. The dashed lines correspond to a thermal source at midrapidity with two temperatures (left) and one temperature (right).

Fig. 2.2 shows rapidity density distributions $\mathrm{dN} / \mathrm{dy}^{(0)}$ of charged pions for very central collisions (corresponding to a cross section of $100 \mathrm{mb}$ ) of $\mathrm{Au}+\mathrm{Au}$ at $1.06 \mathrm{AGeV} 20$ and for positively charged kaons in $\mathrm{Ni}+\mathrm{Ni}$ at $1.93 \mathrm{AGeV}$ 21]. The data are measured in the backward hemisphere only and symmetrized with respect to midrapidity $\left(\mathrm{y}^{(0)}=\mathrm{y} / \mathrm{y}_{\text {proj }}\right.$ with $\mathrm{y}_{\text {proj }}$ being the center-of-mass rapidity of the projectile and $\mathrm{y}^{(0)}=0$ at midrapidity).

The distributions have a gaussian-like shape. For the pions the full width at half height is approximately a factor of 1.8 larger than the beam rapidity $\left(\mathrm{y}^{\text {beam }}=0.67\right.$ in the c.m.s. at $1 \mathrm{AGeV}$ ). This pertains also to central $\mathrm{Ni}+\mathrm{Ni}$ collisions at $1.93 \mathrm{AGeV}$ (not shown). The corresponding number for $\mathrm{K}^{+}$is about 1.2 for $\mathrm{Ni}+\mathrm{Ni}$ collisions at $1.93 \mathrm{AGeV}$.

For the $\mathrm{Ni}+\mathrm{Ni}$ system, the width of the pion and kaon rapidity distributions is in fairly good agreement with the assumption of an isotropically emitting thermal source [17, 21, 22. For Au+Au collisions at 1.06 $\mathrm{AGeV}$, the width of the pion rapidity distribution is found to be slightly narrower than expected for an isotropic thermal source located at midrapidity (dashed lines: fit with two temperatures). For a detailled discussion of the pion data see section 3.2.

Fig. 2.3 shows the rapidity distributions of pions, kaons, protons and Lambdas measured in central $\mathrm{Au}+\mathrm{Au}$ collisions at $10 \mathrm{AGeV}$ 23, 24, 25, 26, 27]. All distributions have a full width at half maximum around 2.2 units of rapidity which translates into a width of $\delta \mathrm{y}^{(0)}=1.4$, except for the negatively charged kaons for which the width comes out smaller by $20 \%$ (see Table 2.1). The widths of the rapidity distributions are larger than expected for an isotropic thermal source with a temperature of $\mathrm{T}=0.13 \mathrm{GeV}$ (dashed lines in Fig. 2.3). The data (except for $\mathrm{K}^{-}$) can be reproduced assuming a longitudinally expanding source with an average expansion velocity of $\left\langle\beta_{l}\right\rangle=0.5$ and a temperature of $\mathrm{T}=0.13 \mathrm{GeV}$ [23]. This corresponds to local isotropic sources distributed over a rapidity interval of \pm 1.1 units around midrapidity. It is interesting to note that the widths of the pion and kaon rapidity distributions are independent of the system size (see Table 2.1]). The values found for $\mathrm{Si}+\mathrm{Al}$ collisions at $13.7 \mathrm{AGeV}[28$ and even for $\mathrm{p}+\mathrm{p}$ collisions at $12 \mathrm{AGeV}$ 

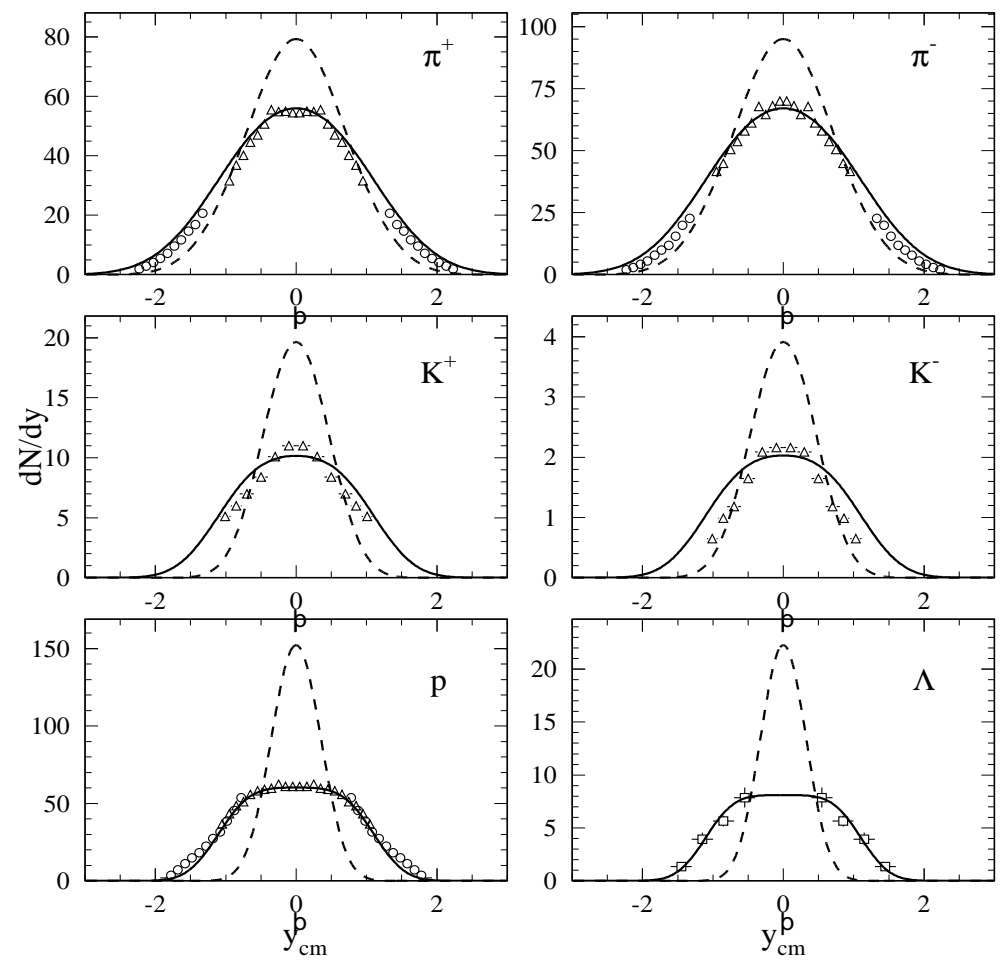

Figure 2.3: Rapidity distributions of various particles as measured in central $\mathrm{Au}+\mathrm{Au}$ collisions at 10 AGeV. Dashed lines: isotropic thermal distribution for $\mathrm{T}=0.13$ $\mathrm{GeV}$. Solid lines: distributions for a source at the same temperature expanding with $<\beta_{l}>=$ 0.5 . The picture is taken from 23, the data are from $[24,25,26,27$.

[29] are within errors the same.

The situation is similar at SPS energies. Fig. 2.4 shows the rapidity distributions of negatively charged particles (mostly pions with an admixture of $8 \% \mathrm{~K}^{-}$and $2 \% \bar{p}$ ) measured in central $\mathrm{Pb}+\mathrm{Pb}(\mathrm{S}+\mathrm{S}$ ) collisions at 158 (200) AGeV by the NA49 (NA35) collaboration together with data from nucleon-nucleon collisions [30]. The agreement of nucleon-nucleon and nucleus-nucleus data excludes collective flow to be the reason for the large width of the rapidity distribution.

Fig. 2.5 shows the rapidity distributions for kaons measured in central $\mathrm{Pb}+\mathrm{Pb}$ collisions at $158 \mathrm{AGeV}$ compared to the kaon distributions from $\mathrm{S}+\mathrm{S}$ at $200 \mathrm{AGeV}$ [31]. The kaon data from $\mathrm{Pb}+\mathrm{Pb}$ (which have large systematical errors) fall out of the general systematics which says that all widths of the kaon distributions are only slightly smaller than those of the pions and that the widths are independent of the size of the colliding system.

The results for the widths of the rapidity distributions are summarized in table 2.1 and Fig. 2.6 in which for comparison the data from nucleon-nucleon $(\mathrm{p}+\mathrm{p})$ interactions have been added. Except for the lowest (SIS) energies the width of all rapidity distributions are wider than expected for an isotropically emitting thermal system. From Fig. 2.6 one concludes that the widths of the rapidity density distributions scale with beam rapidity both for pions and kaons. The spread of the rapidity distribution measured in units of c.m. beam rapidity shows a small but continuous decline from 1.8 at low (SIS) energies to 1.2 at high (SPS) energies (see Table 2.1). The corresponding number for the high energy data from the microscopic transport model UrQMD is 1.1 [32]. It will be interesting to see whether this model also finds the same trend as a function of energy.

At SPS energies the distribution of the produced particles is twice as wide as expected from a thermal fireball. This longitudinal boost of the pions and kaons from $\mathrm{A}+\mathrm{A}$ collisions is very similar to what is observed in $\mathrm{p}+\mathrm{p}$ interactions. In fact at the highest energy the latter exhibits even a slightly larger width than what is obtained from nucleus-nucleus collisions.

The longitudinal boost in $\mathrm{p}+\mathrm{p}$ interactions was explained by the formation and fragmentation of strings which are the dominant source of new particles. The fact that a very similar boost is observed in nuclear collisions at the same energy suggests that the longitudinal distribution of the strings is the same in $\mathrm{A}+\mathrm{A}$ 


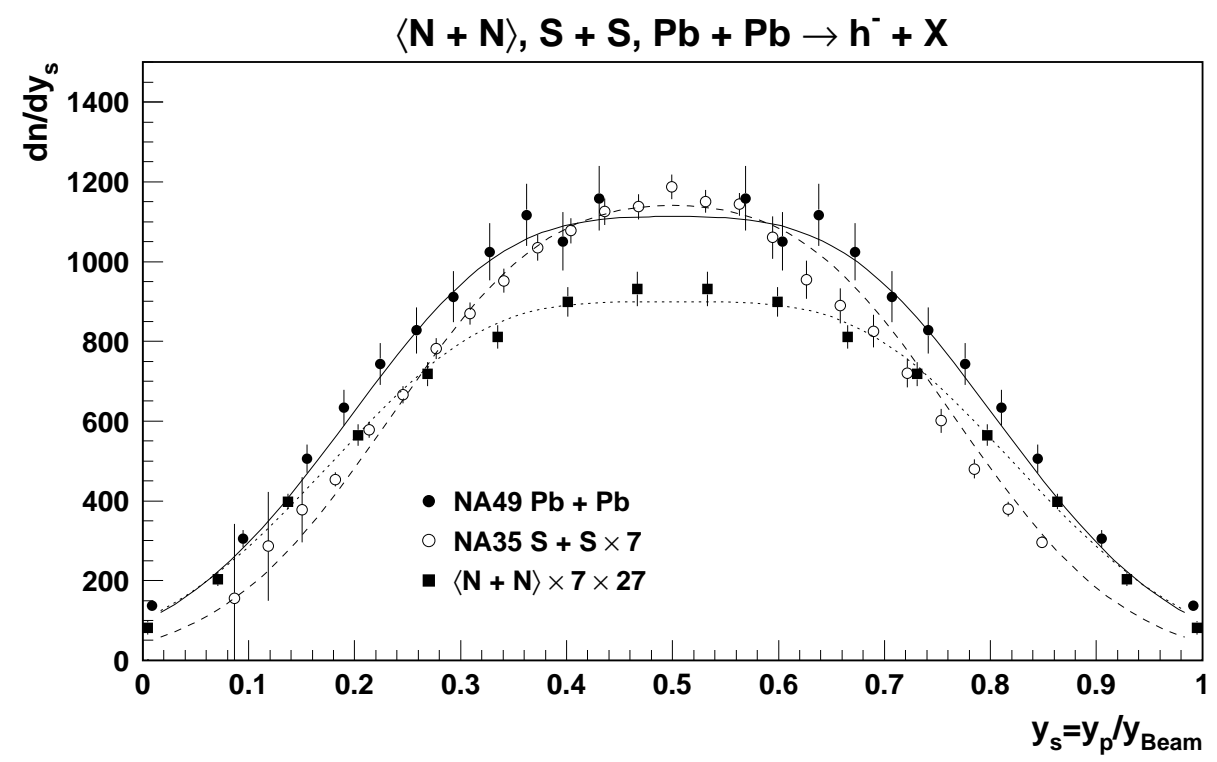

Figure 2.4: Rapidity density distributions of negatively charged hadrons from central $\mathrm{Pb}+\mathrm{Pb}$ at 158 $\mathrm{AGeV}$ (full dots), S+S at $200 \mathrm{AGeV}$ collisions (open dots) together with minimum bias nucleon-nucleon interactions (full squares). The $\mathrm{Pb}+\mathrm{Pb}$ and $\mathrm{N}+\mathrm{N}$ data are reflected around midrapidity, the $\mathrm{S}+\mathrm{S}$ data are not. The curves (gaussian shape for $\mathrm{S}+\mathrm{S}$, double gaussian for $\mathrm{Pb}+\mathrm{Pb}$ and $\mathrm{N}+\mathrm{N}$ ) are meant to guide the eyes. The picture is taken from [30].

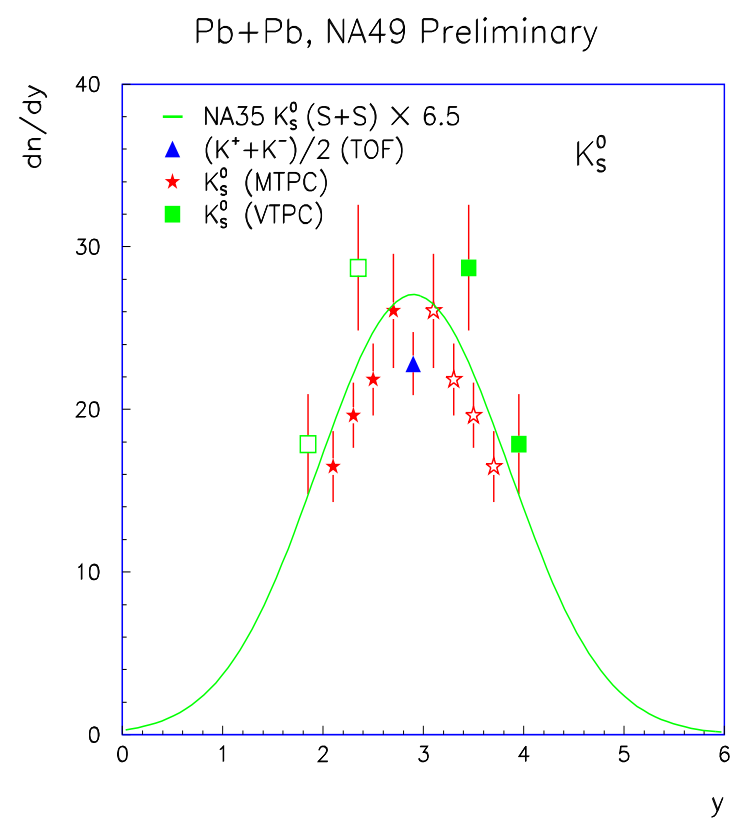

Figure 2.5: Rapidity density distributions of kaons from central $\mathrm{Pb}+\mathrm{Pb}$ collisions at 158 AGeV (symbols, preliminary) compared to the kaons measured in $\mathrm{S}+\mathrm{S}$ collisions at $200 \mathrm{AGeV}$ (line). The data are reflected around midrapidity. The figure is taken from [31. 


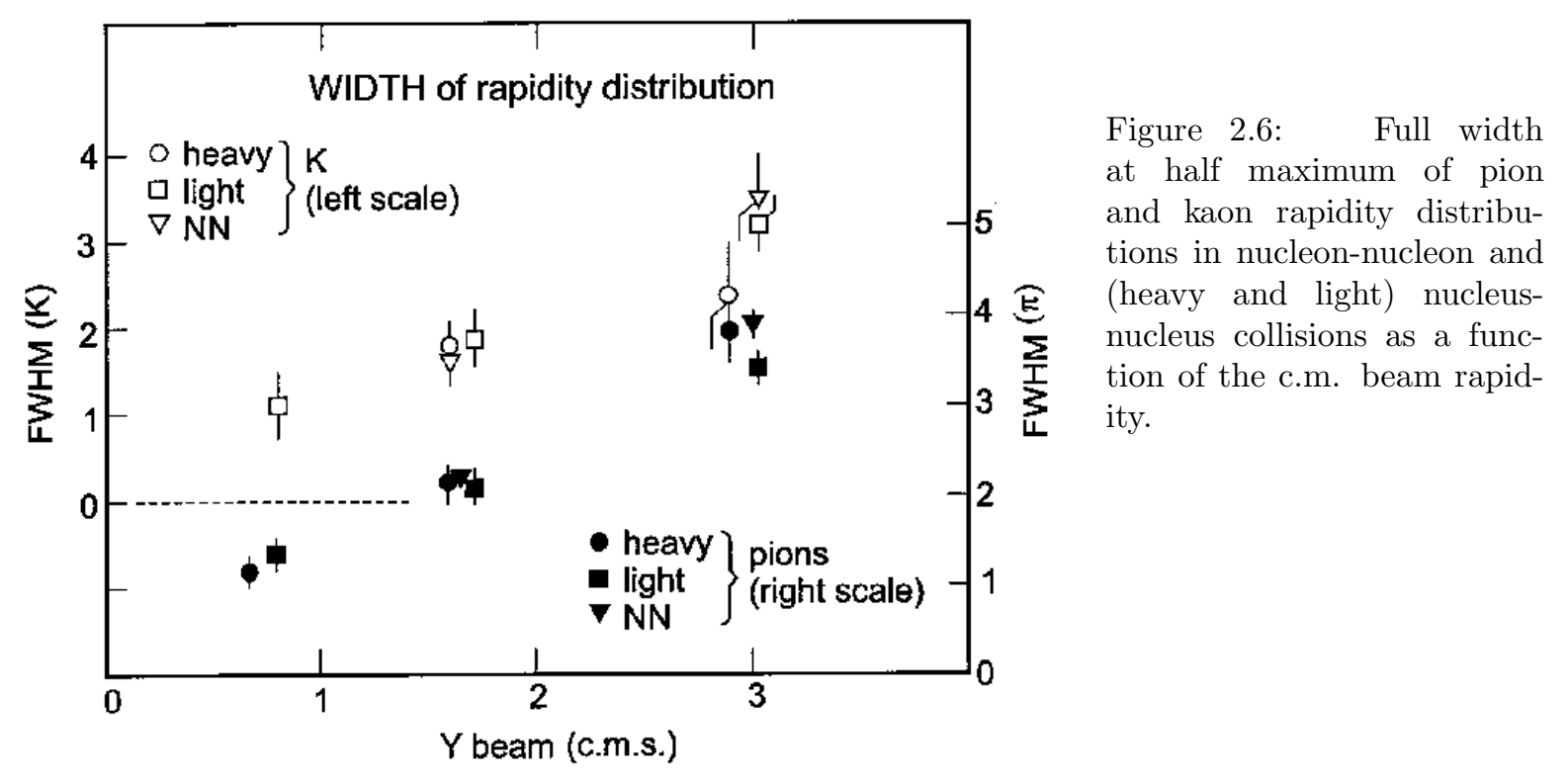

Table 2.1: Widths (FWHM) of rapidity distributions of $\pi$ - and K-Mesons in central nucleus-nucleus and nucleon-nucleon collisions. The $158 \mathrm{AGeV} \mathrm{Pb}+\mathrm{Pb}$ data are preliminary; $\mathrm{N}+\mathrm{N}$ data at $200 \mathrm{GeV}$ are $\mathrm{K}_{s}^{0}$ only. $\delta y^{(0)}$ is the width in units of the beam rapidity in the c.m. system.

\begin{tabular}{|c|c|c|c|c|c|c|}
\hline beam energy & system & $\begin{array}{c}\pi \\
\delta \mathrm{y}^{(0)}\end{array}$ & $\begin{array}{l}\pi \\
\delta \mathrm{y}\end{array}$ & $\begin{array}{c}\mathrm{K} \\
\delta \mathrm{y}^{(0)}\end{array}$ & $\begin{array}{l}\mathrm{K} \\
\delta \mathrm{y}\end{array}$ & Ref. \\
\hline$\overline{1 \mathrm{AGeV}}$ & $\overline{\mathrm{Au}+\mathrm{Au}}$ & $\overline{1.8}$ & $\overline{\overline{1.2}}$ & & & {$[20$} \\
\hline $1.93 \mathrm{AGeV}$ & $\mathrm{Ni}+\mathrm{Ni}$ & 1.8 & 1.6 & 1.2 & 1.07 & \begin{tabular}{|l|l|}
22 & 21 \\
\end{tabular} \\
\hline $10 \mathrm{AGeV}$ & $\mathrm{Au}+\mathrm{Au}$ & 1.37 & 2.2 & 1.12 & $1.8 \pm 0.3$ & 24,26 \\
\hline $12 \mathrm{AGeV}$ & $p+p$ & 1.37 & $2.2 \pm 0.1$ & 1.02 & $1.6 \pm 0.2$ & |29 \\
\hline 13.7. AGeV & $\mathrm{Si}+\mathrm{Al}$ & 1.22 & $2.1 \pm 0.1$ & 1.1 & $1.9 \pm 0.3$ & $\left(\mathrm{~K}^{0}\right) \mid 28$ \\
\hline $13.7 \mathrm{AGeV}$ & $\mathrm{Si}+\mathrm{Al}$ & & & & $2.0 \pm 0.3$ & $\left(\mathrm{~K}^{+}\right)$ \\
\hline $13.7 \mathrm{AGeV}$ & $\mathrm{Si}+\mathrm{Al}$ & & & & $1.6 \pm 0.3$ & $\left(\mathrm{~K}^{-}\right) \sqrt{28}$ \\
\hline $158 \mathrm{AGeV}$ & $\mathrm{Pb}+\mathrm{Pb}$ & 1.3 & $3.8 \pm 0.4$ & 0.76 & $2.4 \pm 0.6$ & 33 \\
\hline $200 \mathrm{AGeV}$ & $\mathrm{S}+\mathrm{S}$ & 1.13 & $3.4 \pm 0.2$ & 1.06 & $3.0 \pm 0.3$ & 34.35 \\
\hline $200 \mathrm{GeV}$ & $\mathrm{N}+\mathrm{N}$ & 1.3 & $3.9 \pm 0.2$ & 1.12 & $3.4 \pm 0.5$ & 36,37 \\
\hline $\mathrm{T}=160 \mathrm{MeV}$ & thermal & & 1.75 & & 1.1 & \\
\hline
\end{tabular}


and $\mathrm{p}+\mathrm{p}$ collisions. Any additional decompressional flow will have only a minor impact on the widths of the rapidity distributions. It will, however, modify significantly the transverse momentum distributions as will be discussed in the following section.

\subsection{Transverse momentum distributions}

Transverse momenta of particles produced in hadronic interactions are one of the measures of the internal energy of the system. However, quantitative comparisons must pay attention to the proper definition of the transverse directions. Normally they are defined with respect to the beam direction. This may not always be appropriate 2 . In the case of a single source emitting particles according to a thermal spectrum the kinetic energies are Boltzmann distributed. This scenario seems to be adequate for low energy nuclear collisions.

For high-energy nucleon-nucleon and nucleus-nucleus collisions Bjorken proposed a longitudinal expanding boost-invariant reaction zone [38]. In this case the transverse momentum spectrum is independent of rapidity and the shape of the invariant cross section as a function of transverse momentum coincides with the one obtained from a single fireball (at the same temperature) integrated over all rapidities. If the kinetic energy (in the transverse direction) of the hadrons is given not only by the thermal motion but also by collective effects like flow, the magnitude and the shape of the transverse momentum distribution may change but still reflect the total kinetic (transverse ) energy. In such a case and for transverse momenta between approximately $0.5 \times \mathrm{m}_{0} \times \mathrm{c}$ and $5 \times \mathrm{m}_{0} \times \mathrm{c}$ a single slope is in general a good approximation but it will be different for different particle masses $\left(\mathrm{m}_{0}=\right.$ rest mass, $\mathrm{c}=$ velocity of light).

In the following paragraph we avoid the problem of nonthermal slopes by using the average momentum as a measure of the energy in the transverse degrees of freedom. For heavy particles this quantity is not always available because of inclomplete coverage of $\mathrm{p}_{T}$. In such cases the average $\mathrm{p}_{T}$ is derived from the slopes, since for heavy particles a single slope seems to describe well the transverse mass spectra.

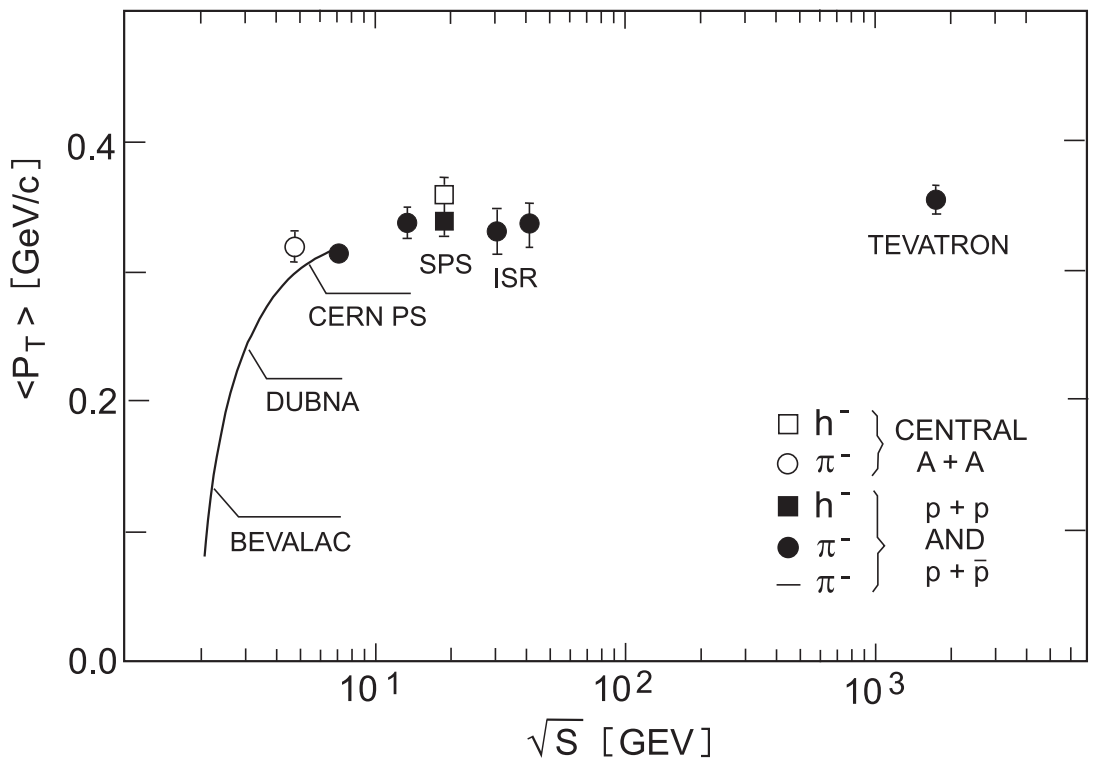

Figure 2.7: Average transverse momenta of negatively charged hadrons produced in $\mathrm{p}+\mathrm{p}$, $\mathrm{p}+\bar{p}$ and $\mathrm{A}+\mathrm{A}$ collisons as function of $\sqrt{s}$.

Fig. 2.7 summarizes the average transverse momenta of negatively charged hadrons measured in hadronic interactions. The average transverse momenta of $\pi^{-}$produced in $\mathrm{p}+\mathrm{p}(\mathrm{p}+\bar{p})$ interactions rise steeply with $\sqrt{s}$ from low (BEVALAC/SIS) to medium (AGS) energies with a saturation beyond. In fact the mean transverse momentum of negatively charged pions remains constant within some ten $\mathrm{MeV} / \mathrm{c}$ above $\sqrt{s}=10$ $\mathrm{GeV}$ as depicted in Fig. 2.7. This value corresponds to a temperature of $140-150 \mathrm{MeV}$ in a thermal picture

\footnotetext{
${ }^{2}$ For example in hadronic interactions involving hard parton scattering the source of the hadrons may be the jet in the wake of the leading quark. The transverse momenta of those hadrons are then the components perpendicular to the jet axis
} 


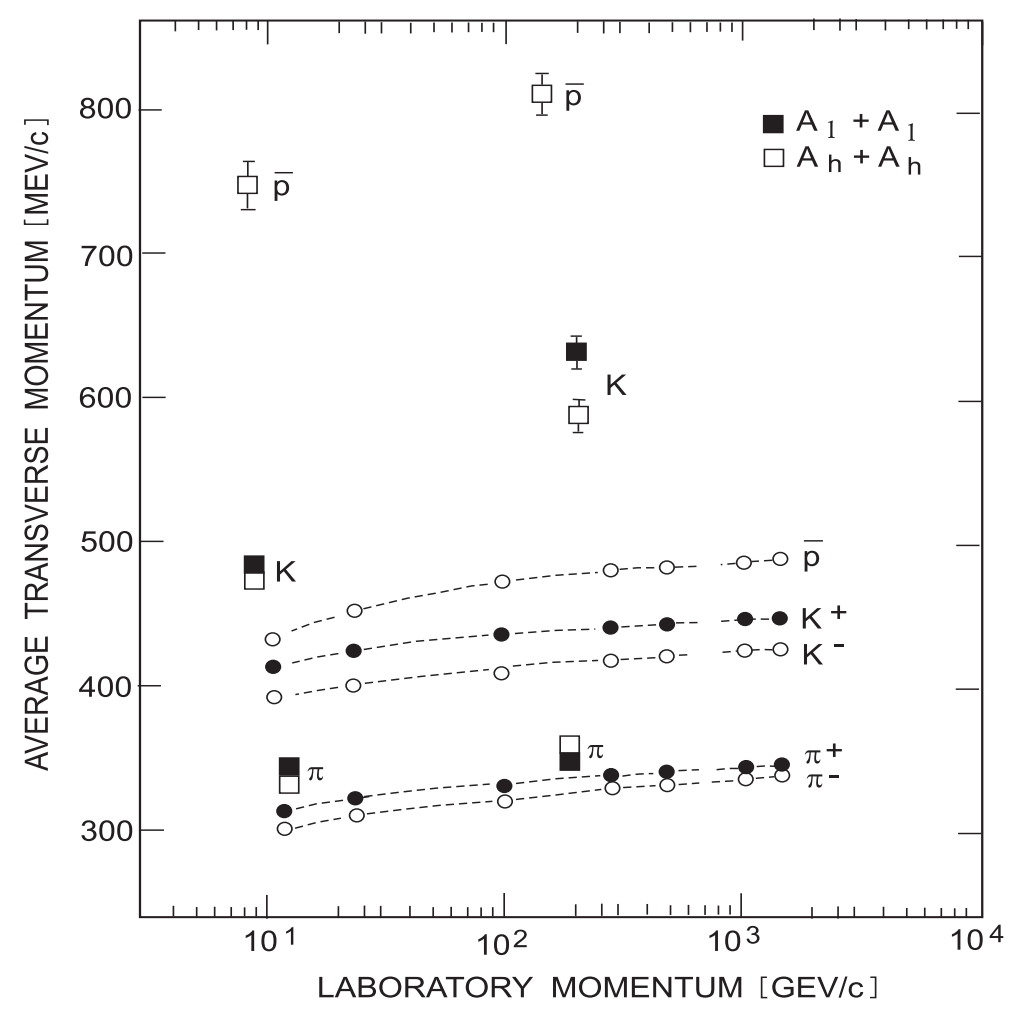

Figure 2.8: Average transverse momenta of different particle species as function of beam energy for $p+p$ interactions (circles) 43 and nucleus-nucleus collisions (squares, l=light, $\mathrm{h}=$ heavy).

and is consistent with Hagedorn's temperature limit for hadronic fireballs [39]. The comparison with $A+A$ collisions is straight forward at AGS energies for which the average transverse momenta of pions have been determined in full phase space [24]. The value of $320 \pm 5 \mathrm{MeV} / \mathrm{c}$ for $\left\langle p_{T}\right\rangle$ of all pions in central ${ }^{197} A u+{ }^{197} A u$ collisions at $\sqrt{s}=5 \mathrm{GeV}$ falls slightly above the universal curve in Fig. 2.7 which passes through $300 \mathrm{MeV} / \mathrm{c}$ at $\sqrt{s}=5 \mathrm{GeV}$. At SPS energies results on negatively charged particles in central ${ }^{32} S+{ }^{32} S$ collisions 40, 41 give $\left\langle p_{T}\right\rangle=355 \pm 5 \mathrm{MeV} / \mathrm{c}$ which is again somewhat higher than the universal curve at $\sqrt{s}=20 \mathrm{GeV}(330 \mathrm{MeV} / \mathrm{c})$. The increase is partly due to the admixture of $K^{-}$and $\bar{p}$. Recent measurements of negatively charged particle spectra in central ${ }^{208} \mathrm{~Pb}+{ }^{208} \mathrm{~Pb}$ collisions at the SPS yield a similar value for $\left\langle p_{T}\right\rangle$ of approximately $360 \mathrm{MeV} / \mathrm{c}$ [42]. In a thermal picture the difference of $20 \mathrm{MeV} / \mathrm{c}$ in $\left\langle p_{T}\right\rangle$ corresponds to a difference in temperature of only $10 \mathrm{MeV}$ or $7 \%$. In terms of energy (stored in the pions) the higher $\left\langle p_{T}\right\rangle$ in $A+A$ collisions amounts to an increase of the average transverse energy of only $5 \%$. This means that the pion emission characteristics are similar in $p+p$ and $A+A$ collisions. At AGS and SPS energies, the observable $\left\langle p_{T}>\right.$ seems to be insensitive to the environment the pions are emitted from.

The systematics of the average transverse momenta of different particle species as function of beam energy (above $10 \mathrm{GeV} / \mathrm{c}$ beam momentum) is shown in Fig. 2.8 for $\mathrm{p}+\mathrm{p}$ interactions 43 and nucleusnucleus collisions. The entries in the figure for the latter were derived from measurements near central rapidity. It is evident that the mean transverse momenta of the pions show only a slight increase with beam energy. However, the heavier particles experience a boost in transverse direction when going from $\mathrm{p}+\mathrm{p}$ to $\mathrm{A}+\mathrm{A}$ collisions. The mean transverse momenta increase with particle mass, size of the colliding system and beam energy.

The same picture emerges if the transverse mass spectra $\left(\mathrm{m}_{T}=\sqrt{m_{0}^{2}+p_{T}^{2}}\right)$ are analysed. Fig. 2.9 shows transverse mass $\left(\mathrm{m}_{T}-\mathrm{m}_{0}\right)$ distributions for charged pions, kaons and antiprotons from $\mathrm{Pb}+\mathrm{Pb}$ central collisions at $158 \mathrm{AGeV}$ bombarding energy [44]. The spectra can be parameterized by exponential functions (dashed lines) except for the low $\mathrm{m}_{T}$ pions. In this kinematical region, resonance decays are expexted to contribute to the pion yield. Therefore, the pion spectra were fitted above $\mathrm{m}_{T}=0.2 \mathrm{GeV}$ only. The resulting inverse slope parameters increase with increasing particle mass. 


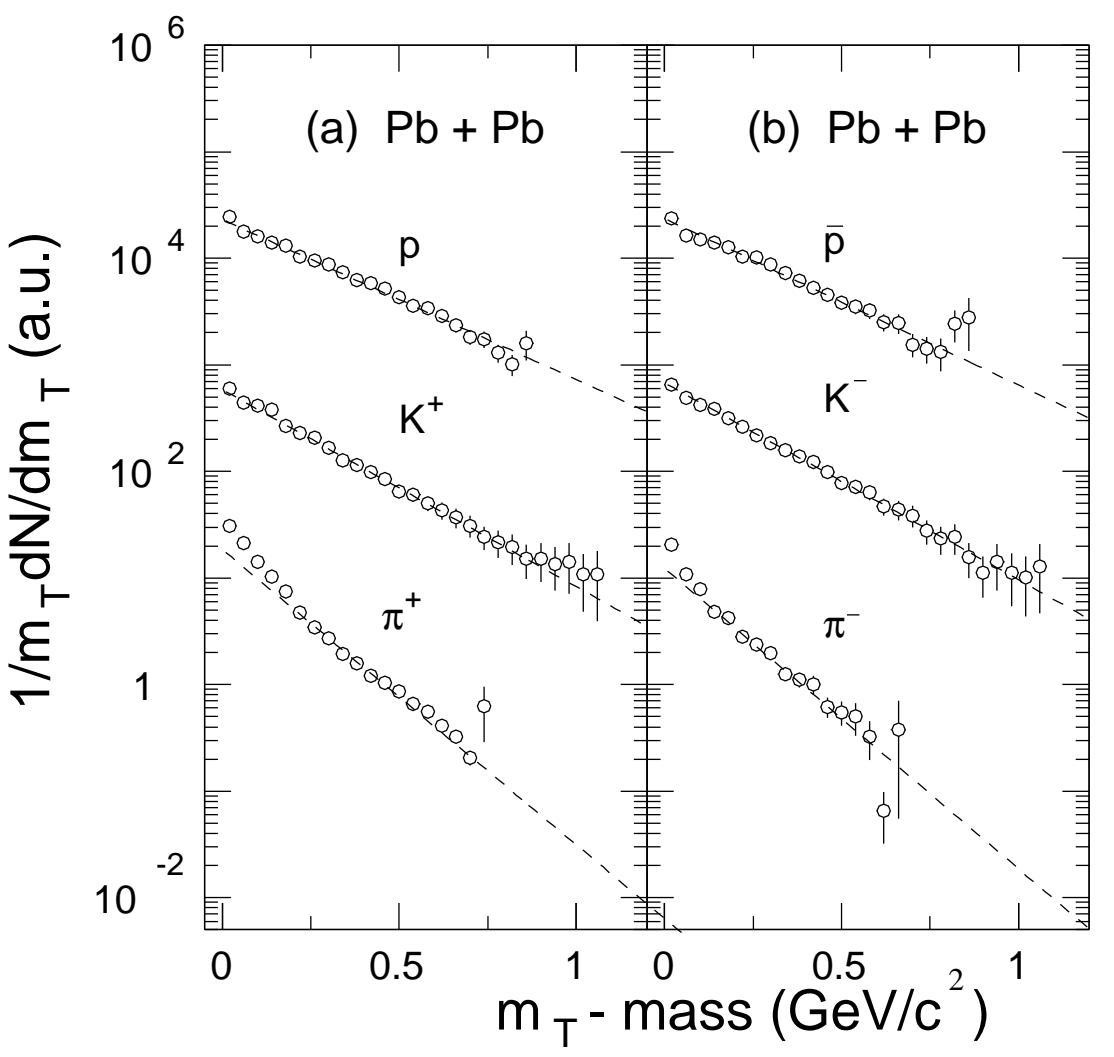

Figure 2.9: Transverse mass distributions for positive (left) and negative particles (right) from $\mathrm{Pb}+\mathrm{Pb}$ central collisions at 158 AGeV bombarding energy [44. The spectra are described by exponential functions (dashed lines).

In Fig. 2.10 the inverse slope parameters of pions, kaons and antiprotons from $\mathrm{Pb}+\mathrm{Pb}$ collisions $(\sqrt{s}=17.3 \mathrm{AGeV})$ are compared to those from $\mathrm{S}+\mathrm{S}(\sqrt{s}=19.4 \mathrm{AGeV})$ and $\mathrm{p}+\mathrm{p}$ collisions $(\sqrt{s} \approx 23 \mathrm{AGeV})$ 44. In the case of $\mathrm{p}+\mathrm{p}$ the inverse slope parameter $\mathrm{T}$ is constant for the different particle species whereas for nucleus-nucleus collisions $T$ increases as the mass of the particle and the mass of the collision system increases 3 . This characteristic behavior suggests that the transverse motion has a component which behaves like a velocity rather than a momentum.

There are two scenarios in which the observed effect is present: (i) initial state scattering which broadens the effective distribution of beam directions thus introducing a mass-dependent transverse momentum component of the produced particles and (ii) the build-up of pressure with its decompressional flow which induces a velocity field. In this hydrodynamical description the reaction volume expands after compression and the nuclear matter, i.e. all particles, flow with the same collective velocity (on top of their thermal motion). Hence particles with higher masses will have higher energies. In collisions between very heavy nuclei, multiple scattering becomes more important which results in a higher nuclear stopping and a stronger collective flow in the expanding system. Therefore, the collective motion will be more pronounced in the $\mathrm{Pb}+\mathrm{Pb}$ than in the $\mathrm{S}+\mathrm{S}$ system and will vanish for $\mathrm{p}+\mathrm{p}$ collisions.

It has been shown for CERN-SPS energies that initial scattering may also account for the observed transverse momentum spectra of pions and nucleons from proton-nucleus and nucleus-nucleus collisions 45. On the other hand, the hydrodynamical picture describes well the inverse slope parameters of particles and light fragments from nucleus-nucleus collisions at LBL/SIS and AGS all the way up to CERN-SPS energies. Therefore we analyze in the following the transverse momentum distributions of $\mathrm{A}+\mathrm{A}$ collisions in terms of the hydrodynamical model.

\footnotetext{
${ }^{3}$ we use $\mathrm{T}$ for the inverse slope parameter because sometimes it is identical to the temperature. Whenever $\mathrm{T}$ appears as a subscript it denotes the transverse direction
} 


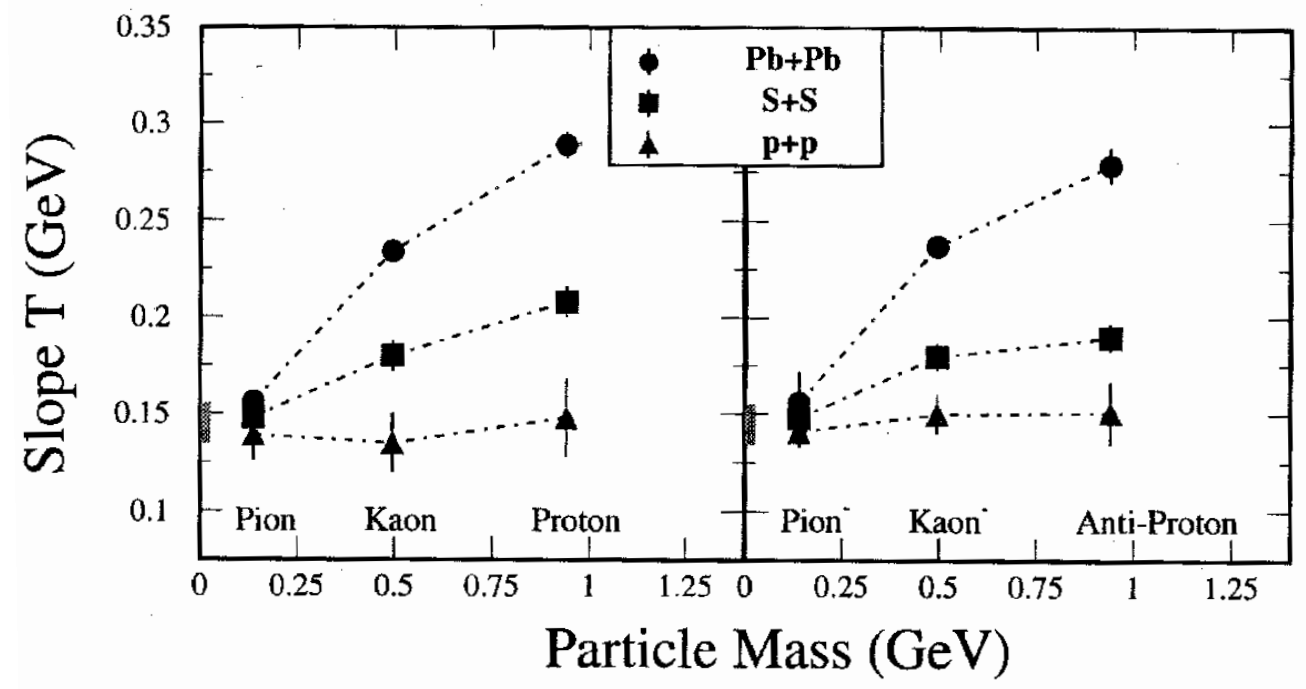

Figure 2.10: Inverse slope parameters for positive (left) and negative particles (right) from $\mathrm{Pb}+\mathrm{Pb}$ collisions $(\sqrt{s}=17.3 \mathrm{AGeV})$ in comparison to those from $\mathrm{S}+\mathrm{S}(\sqrt{s}=19.4 \mathrm{AGeV})$ and $\mathrm{p}+\mathrm{p}$ collisions $(\sqrt{s} \approx 23 \mathrm{AGeV})$ 44 .

Using the model of Ref. [46], the transverse momentum distributions are calculated according to

$$
\frac{d N}{m_{T} d m_{T}} \propto \int_{0}^{R} r d r m_{T} I_{o}\left[\frac{p_{T} \cosh (\rho)}{T_{o}}\right] \times K_{1}\left[\frac{m_{T} \cosh (\rho)}{T_{o}}\right]
$$

with $\rho=\tanh ^{-1} \beta_{T}$, the transverse velocity profile $\beta_{T}(r)=\beta_{T}^{\max }(r / R)$ and Bessel functions $I_{o}, K_{1}$. The two free parameters $T_{0}$ and $\beta_{T}^{\max }$ determine the shape of the $m_{T}$ spectra. In general, the fits to the experimental data show an anticorrelation of these two parameters. Temperatures in the range from 100 to $200 \mathrm{MeV}$ together with appropriate flow parameters describe well the data for pions, kaons and protons. Assuming a value of $T_{0}=140 \mathrm{MeV}$, the maximum transverse velocities are found to be $\beta_{T}^{\max }=0.41 \mathrm{c}$ and $0.6 \mathrm{c}$ for $\mathrm{S}+\mathrm{S}$ and $\mathrm{Pb}+\mathrm{Pb}$ collisions, respectively. These values correspond to average transverse flow velocities of $0.27 \mathrm{c}$ and $0.4 \mathrm{c} 44$.

Similar values both for the intrinsic temperature and the transverse particle velocity have been found in nucleus-nucleus collisions at AGS energies. Fig. 2.11 shows transverse mass spectra of $\pi^{+}, \mathrm{K}^{+}$, $\mathrm{p}$ and $\mathrm{d}$ measured in $\mathrm{Si}+\mathrm{Au}$ collisions at $14.6 \mathrm{AGeV} / \mathrm{c}$ 14, 28]. The solid lines are results of calculations using equ.2.3. In order to avoid complications due to resonance decays, the fit was restricted to $\left(m_{T}-m_{o}\right)>0.3$ $\mathrm{GeV} / \mathrm{c}^{2}$. Fig. 2.11 demonstrates that a good agreement between data and calculations is obtained for $T_{o}=0.12(0.14)$ and $\beta_{T}^{\max }=0.58(0.50)$ corresponding to average velocities of $\left\langle\beta_{T}>=0.39(0.33)\right.$.

At LBL/SIS energies, a transverse (azimuthally symmetric) flow component was observed by measuring light fragment spectra in very central $\mathrm{Au}+\mathrm{Au}$ collisions [47, 48]. It was found that the collective expansion uses about $50 \%$ of the available energy [6, 47. The analysis of transverse mass spectra of light fragments using a blast wave model 49 yields a temperature of $T_{o}=81 \pm 24 \mathrm{MeV}$ and a constant radial velocity of $\beta=0.32 \pm 0.05$ for central $\mathrm{Au}+\mathrm{Au}$ collisions at $1.0 \mathrm{AGeV}$ 477.

Fig. 2.12 shows transverse mass spectra of $\pi^{-}$, p and d measured in central $\mathrm{Ni}+\mathrm{Ni}$ and $\mathrm{Au}+\mathrm{Au}$ collisions at $1.06 \mathrm{AGeV}[17,20,22,50$. It can be seen that (i) the slope parameter increases with particle mass and (ii) this increase is more pronounced for the heavy system. Both observations indicate a collective motion of matter.

In summary, the slope of the transverse mass spectra of emitted particles in a wide range of masses can be successfully explained within a hydrodynamical picture by a freeze-out temperature and a transverse flow velocity $\left(\beta_{T}=\right.$ constant or $\beta_{T} \propto \mathrm{r} / \mathrm{R}$ ). Fig. 2.13 presents the excitation function of these parameters with respect to the bombarding energy [44]. The effect of resonance formation and decay is neglected in this picture. For its influence see for example reference [32]. 

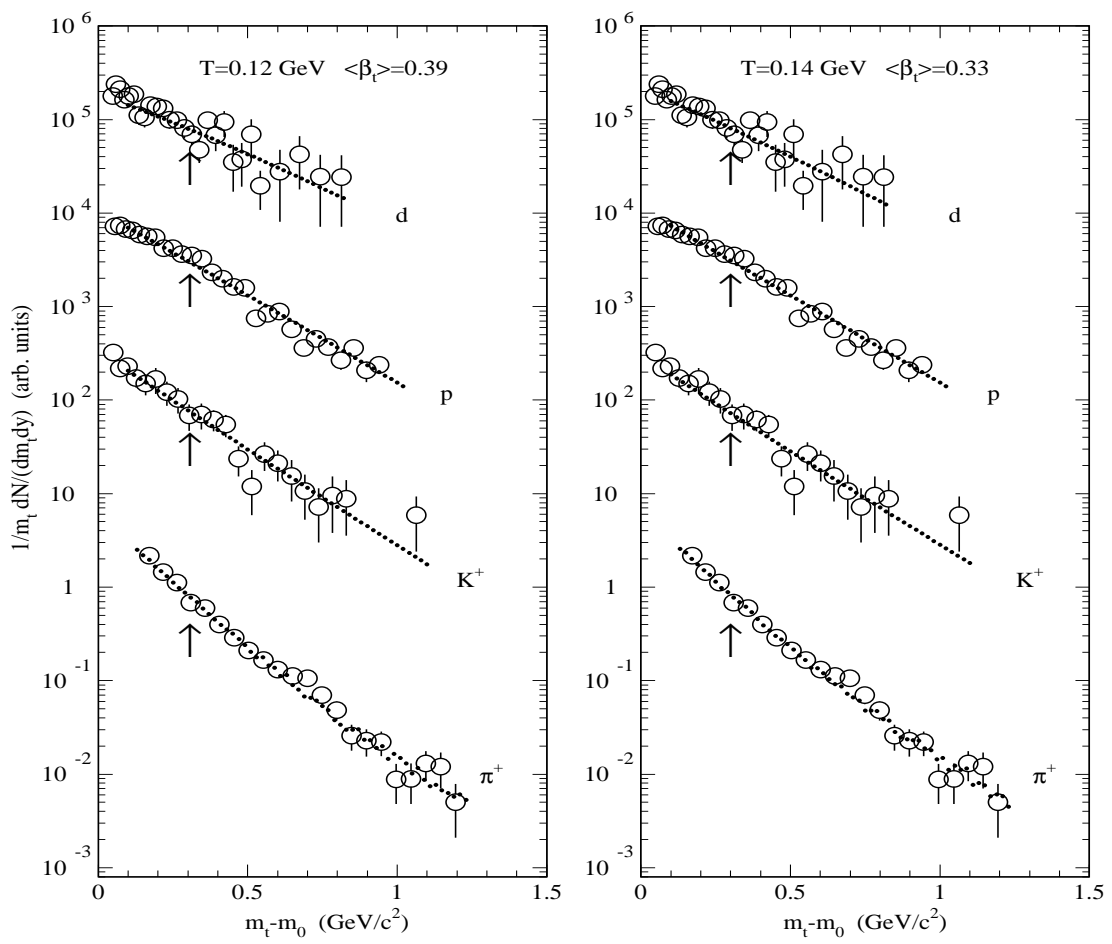

Figure 2.11: Transverse mass spectra of $\pi^{+}, \mathrm{K}^{+}, \mathrm{p}$ and d measured in $\mathrm{Si}+\mathrm{Au}$ collisions at $14.6 \mathrm{AGeV} / \mathrm{c}$ 14, 28. The solid lines are results of calculations using equ. 2.3 with $\mathrm{T}=$ $0.12 \mathrm{GeV}$ and $\left\langle\beta_{l}>=\right.$ 0.39 (left) and $\mathrm{T}=0.14$ $\mathrm{GeV}$ and $\left\langle\beta_{l}>=0.33\right.$ (right). For details see text.

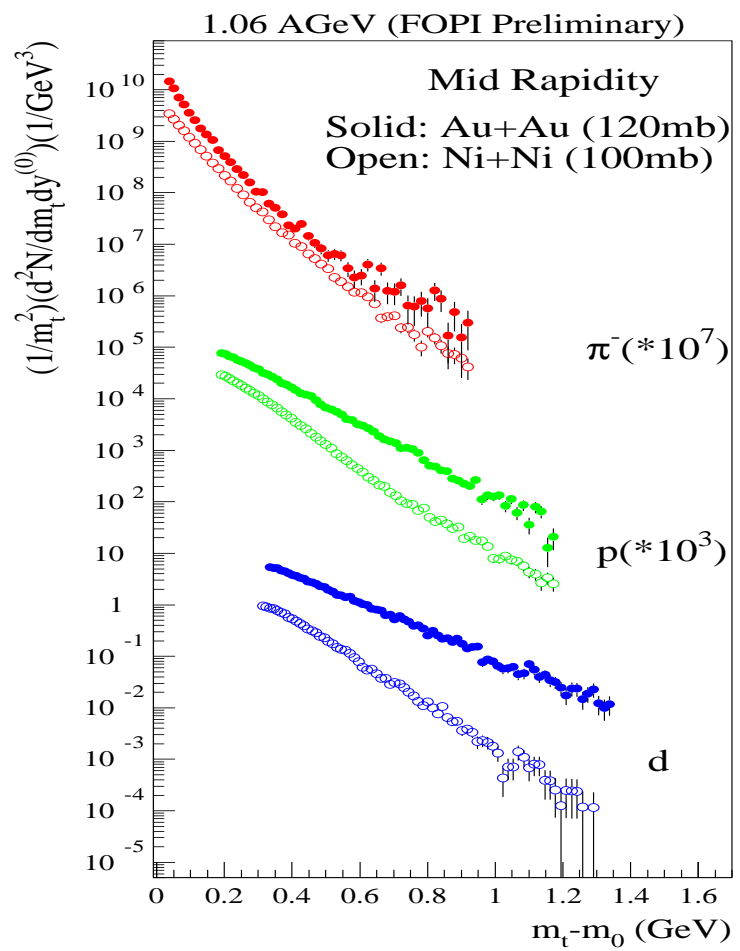

Figure 2.12: Transverse mass spectra of $\pi^{-}, \mathrm{p}$ and $\mathrm{d}$ measured in central $\mathrm{Ni}+\mathrm{Ni}$ (open points) and $\mathrm{Au}+\mathrm{Au}$ collisions (full points) at $1.06 \mathrm{AGeV}$ (preliminary) 20, 22, 17, 50.

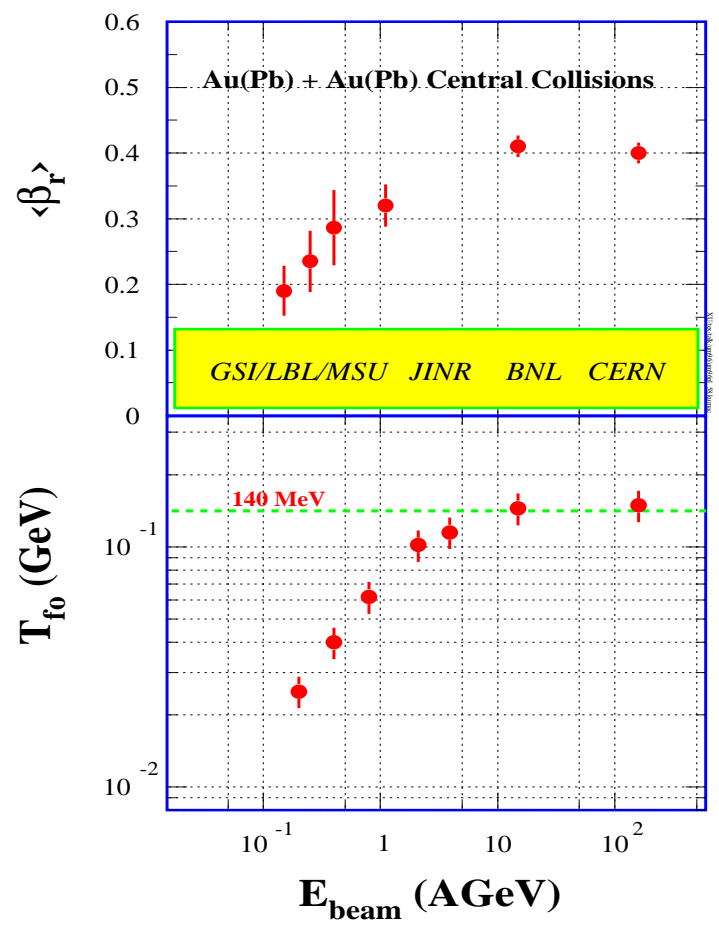

Figure 2.13: Compilation of average flow velocities and inverse slope parameters as a function of bombarding energy (see text). The picture is taken from 44]. 
Both the temperature and the transverse flow velocity exhibit a saturation above AGS energies. the limiting temperature is about $140 \mathrm{MeV}$ again in line with the limit stated by Hagedorn. The average transverse flow velocity increases up to $0.4 \mathrm{c}$ which corresponds to a maximum velocity around $0.6 \mathrm{c}-0.7 \mathrm{c}$.

At SIS energies, particles heavier than pions (such as etas, kaons and antiprotons) can hardly be produced in a first chance nucleon-nucleon collision. The threshold energies for producing a $\eta, \mathrm{K}^{+}, \mathrm{K}^{-}$ and a $\bar{p}$ in a free nucleon-nucleon collision are $1.25 \mathrm{GeV}, 1.58 \mathrm{GeV}, 2.5 \mathrm{GeV}$ and $5.6 \mathrm{GeV}$, respectively. Therefore, their production in nucleus-nucleus collisions at beam energies below these thresholds requires collective and/or medium effects. According to transport models, subthreshold particle production proceeds predominantly via multiple hadron-hadron interactions which occur more frequently in the dense phase of the collision. At this stage, the collective expansion has not developed yet. Those particles which freeze out in this phase should not be affected by the radial motion. Indeed it was found in nucleus-nucleus collisions at $1-2 \mathrm{AGeV}$ that the emitted high-energy pions, kaons, etas and antiprotons exhibit similar slope parameters [51, 52, 53, 54, 55]. These common slope parameters depend on the beam energy and on the mass of the colliding nuclei.

\subsection{Energy in produced particles}

Particle production in hadronic collisions can be studied in various observables. One of them is the multiplicity of different particle species (see chapters 3 and 4). An alternative and more global approach to study regularities in particle production makes use of the inelasticity of a hadronic interaction. Inelasticity is here defined as that fraction of the center-of-mass kinetic energy of the incident nucleons which is deposited in produced particles. There are two ways to measure the corresponding observable:

- The fraction of the energy (measured in the center-of-mass frame) lost by the colliding particles is determined from the difference between the average kinetic energies before and after the collision. This implies the measurement of the kinetic energies of the participating nucleons in the final state of the collision.

- The second, equivalent, measurement compares the total energy of all produced particles with the sum of the kinetic energies of all participating nucleons before the collision. This choice requires identification and the momentum or energy measurement of all produced particles in the final state. With present experiments this is an ambitious task. However, symmetries and regularities often allow to determine the energy carried by all produced particles to a precision of better than $10 \%$.

In the following we will use both prescriptions to determine the inelasticity. Furthermore we will differentiate between the energy transferred to strange and nonstrange particles.

\subsubsection{Inelasticity}

In order to compare nucleon-nucleon and nucleus-nucleus collisions we need to define the particle multiplicity per $\mathrm{N}+\mathrm{N}$ interaction. However, the definition of a $\mathrm{N}+\mathrm{N}$ interaction is ambiguous: should one take into account all (elastic and inelastic) $\mathrm{N}+\mathrm{N}$ collisions or inelastic collisions only? In studies of hadronic particle production usually only inelastic interactions are used. On the other hand it is obvious that even in central nucleus-nucleus collisions some of the nucleons suffer only elastic interactions. Furthermore, the study of particle multiplicities as function of beam energy is more plausible if it is related to all interactions: close to the threshold of pion production in $N+N$ interactions, the pion multiplicity is equal to unity if only inelastic collisions are considered whereas it tends to zero if the total cross section is taken as the reference. Therefore, in the following we will use for the evaluation of inelasticity in $N+N$ interaction the fraction of energy lost by the incident nucleons averaged over all elastic and inelastic strong interactions.

At low energies for which single pion production dominates the inelastic cross section, the inelasticity of $N+N$ collisions is simply given by

$$
<E_{\pi}>\times \sigma_{\text {inel }} / \sigma_{\text {tot }} /\left(2 \times E_{N}^{k i n}\right)
$$

where $\left\langle E_{\pi}>\right.$ is the average c.m. energy of the pion, $\sigma_{\text {inel }}$ the inelastic cross section, $\sigma_{\text {tot }}$ the total (elastic and inelastic) cross section and $E_{N}^{k i n}$ the c.m. kinetic energy of the incident nucleon. For $\left\langle E_{\pi}\right\rangle$ we have used a value of $0.25 \mathrm{GeV}(0.30 \mathrm{GeV})$ at $1.5 \mathrm{GeV} / \mathrm{c}(2.5 \mathrm{GeV} / \mathrm{c})$ laboratory beam momentum [56]. 
At AGS and SPS beam energies (i.e. $12 \mathrm{AGeV}$ and $200 \mathrm{AGeV}$, respectively) we computed the energy in the produced particles from the particle multiplicities in $p+p$ collisions as compiled by Rossi et al. [43]. The numbers were rescaled with the factor $\sigma_{\text {inel }} / \sigma_{\text {tot }}$ for consistency with the low energy data (see beginning of this subsection). The mean particle energies are computed as the sum of the expression

$$
<E>_{y_{i}}=<m_{T}>_{y_{i}} \cdot \cosh \left(y_{i}\right)
$$

evaluated in each rapidity bin. The result for the energy in the produced particles was checked against the energy loss of the incident protons and the two results were consistent to within $10 \%$. No correction was applied for possible effects of the difference in isospin between $p+p$ and $N+N$ interactions.

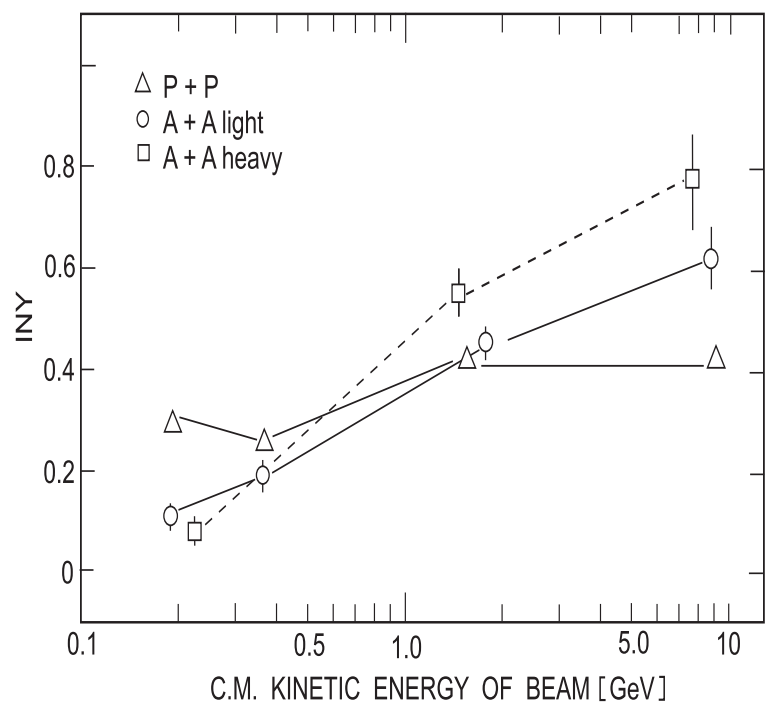

Figure 2.14: Inelasticity of nucleonnucleon and nucleus-nucleus collisions as function of c.m. kinetic energy of the projectile nucleons.

The same procedure was applied to central ${ }^{28} \mathrm{Si}^{27} \mathrm{Al}$ and ${ }^{197} \mathrm{Au}+{ }^{197} \mathrm{Au}$ collisions at AGS energies 24 and central ${ }^{32} S+{ }^{32} S$ collisions at the SPS [40]. For central ${ }^{197} A u+{ }^{197} A u[20]$ and ${ }^{40} \mathrm{Ar}+\mathrm{KCl}$ [68] collisions from SIS/BEVALAC, the energy in the produced particles was calculated from the pion multiplicity and their c.m. energy which was taken as $230(280) \mathrm{MeV}$ at $0.83 \mathrm{GeV}(1.73 \mathrm{GeV})$ kinetic beam energy. Central ${ }^{208} \mathrm{~Pb}+{ }^{208} \mathrm{~Pb}$ collisions at SPS energies were characterized only by the rapidity and $p_{T}$ distributions of the participant protons [57]. The result of all these calculations is shown Fig. 2.14 (an earlier version of this compilation was presented in [58]). The inelasticity is plotted as a function of the center-of-mass beam energy per nucleon for $p+p$, central $A_{l}+A_{l}$ and $A_{h}+A_{h}$ collisions (l=light, $\mathrm{h}=$ heavy). In nucleon-nucleon interactions the inelasticity stays remarkably constant with beam energy from a few $100 \mathrm{MeV}$ to a few $\mathrm{GeV}$, a well established behavior already since a long time: in each interaction the nucleons lose on the average half of their energy. The inelasticity in $p+p$ collisions in Fig. 2.14 is lower than the expected value of $1 / 2$ because the total instead of the inelastic cross section was employed. In contrast to the trend in $N+N$ interactions, nuclear collisions show a significant increase of the inelasticity as a function of the collision energy. This could mean that in a high-energy nucleus-nucleus collision the participating nucleons scatter inelastically more than once. On the other hand, one should keep in mind that the inelasticity is determined mainly by pion production and in chapter 3 we will see that pion reabsorption may be more important at SIS energies than at SPS energies. This effect would also decrease the inelasticity with decreasing beam energy. An observable less affected by reabsorption and thus directly proportional to the inelasticity would be the yield of $\mathrm{K}^{+}$mesons (see chapter 4 ).

Pion reabsorption could also be the origin of the projectile/target mass dependence of the inelasticity. Experiments on pion production in ${ }^{197} \mathrm{Au}+{ }^{197} \mathrm{Au}$ collisions at $1 \mathrm{AGeV}$ find that the number of pions per participating nucleon is about $25 \%$ smaller than in ${ }^{40} \mathrm{Ar}+\mathrm{KCl}$ collisions (see section 3.1). Preliminary results from ${ }^{208} \mathrm{~Pb}+{ }^{208} \mathrm{~Pb}$ collisions at SPS energies indicate that the inelasticity is only slightly higher in the heavy system than in the light ${ }^{32} S+{ }^{32} S$ configuration. This behaviour suggests that pion absorption is correlated with the number of pions per participating nucleon which is 30 times smaller at SIS than at SPS energies. 


\subsubsection{Fraction of energy in strange particles}

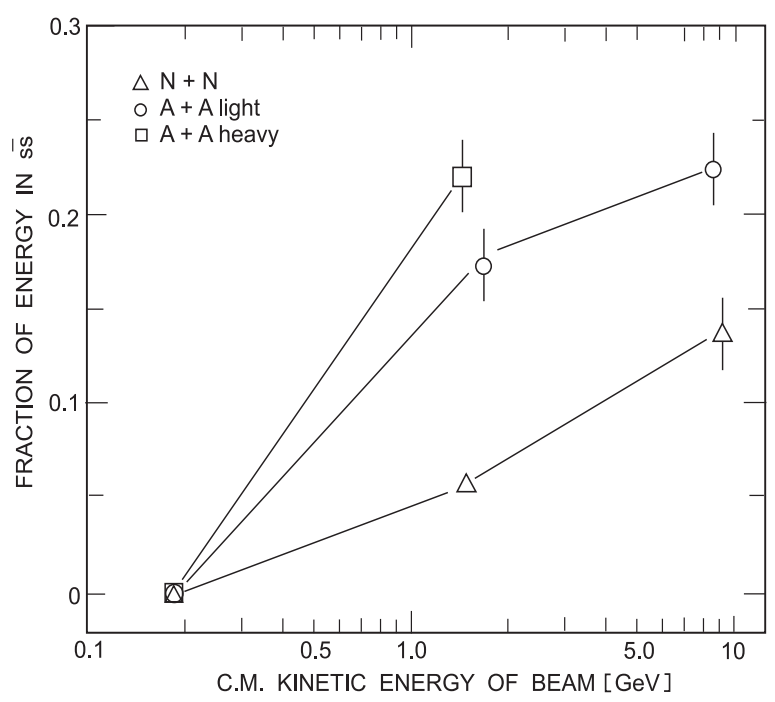

Figure 2.15: Fraction of energy deposited in all strange particles produced in $\mathrm{p}+\mathrm{p}$ and $\mathrm{A}+\mathrm{A}$ (l=light, $\mathrm{h}=$ heavy) collisions as a function of the c.m. kinetic energy of the projectile nucleons.

In this subsection we introduce a new observable which is motivated by two experimental findings: (i) in section 2.2 we have seen that the average transverse momenta of heavy particles (in contrast to pions) increase drastically with system size and (ii) there is the well established phenomenon of the strangeness enhancement in nuclear collisions [59] (see section 4). Both phenomena may be correlated since all strange mesons contain a heavy strange quark. We have computed the energy deposited in the strange particles relative to the total energy in produced particles in order to further quantify the different trends in $N+N$ and $A+A$ collisions. Here again the energy has been computed from the rapidity and $m_{T}$ distributions according to 2.5. $\mathrm{K}_{S}^{0}, \mathrm{~K}^{+}$and $\mathrm{K}^{-}$mesons have been used whenever sufficient spectral information is available. For the hyperons from associated production only a fraction of the total energy has been considered. It has been calculated using the ratio of the difference between the hyperon and the nucleon mass to the nucleon mass. At SPS energies also hyperon pair production has been taken into account. The total energy in the hyperons has been obtained from $\Lambda$ and $\bar{\Lambda}$ particles, which include already $\Sigma^{0}$, by multiplication with a factor of 1.6 to cover also charged hyperons 60 . Fig. 2.15 shows the fraction of energy deposited in all strange particles (FES). As expected this fraction increases with beam energy in $p+p$ interactions in accordance with the trend in the particle multiplicities. In nucleus-nucleus collisions, FES is systematically higher than in $p+p$ interactions. At the lowest energy this is not visible in Fig. 2.15 due to the linear scale of the vertical axis. Around $\sqrt{s}=5 \mathrm{GeV}$, FES increases by a factor 2.4 from $p+p$ to the light collisions system ${ }^{28} \mathrm{Si}+{ }^{27} \mathrm{Al}$. For central ${ }^{197} \mathrm{Au}+{ }^{197} \mathrm{Au}$ collisions an additional increase of $20 \%$ occurs. Such a dependence of FES on the size of the system is weaker than the increase of the number of participant nucleons. Above AGS energies, FES increases with energy less in light nucleus-nucleus than in nucleon-nucleon collisions. This behaviour is mainly due to the fact that the pion yield (i.e. the energy stored in nonstrange particles) increases with beam energy. This increase is stronger for $\mathrm{A}+\mathrm{A}$ than for $\mathrm{p}+\mathrm{p}$ collisions (see Fig. 3.1).

It is an open question whether FES in $\mathrm{A}+\mathrm{A}$ and $\mathrm{N}+\mathrm{N}$ collisions converge to the same universal (hadronic) value. Alternatively, the onset of partonic effects in $\mathrm{A}+\mathrm{A}$ collisions perhaps may lead to a more effective deposition of energy in strange particles. So far the number of strange quarks was used to quantify a possible strangeness enhancement. The relative abundance of s-quarks is the appropriate observable in a situation in which chemical equilibrium governs particle multiplicities whereas we expect the fraction of energy in strange particles to be more sensitive to the dynamics of particle production in the non-equlibrium case. 


\section{Chapter 3}

\section{Pion Production}

Pion production is the dominating inelastic process in nucleus-nucleus collisions. At beam energies of 1-2 $\mathrm{AGeV}$ the pion-to-participant ratio is about $10-20 \%$. In this energy range pions are predominantly created via the excitation and the decay of the $\Delta_{33}$ resonance [61, 62, 63, 64]. At higher bombarding energies, heavier baryonic resonances are excited which also decay predominantly into pions. At beam energies of $5-10 \mathrm{AGeV}$, the pion-to-participant ratio reaches values of $0.7-1$. At ultrarelativistic beam energies (above $100 \mathrm{AGeV}$ ), pions have become the most abundant particle species' with a 10-20\% "contamination" of baryons and K-mesons. The high center-of-mass energy leads to the formation of vector mesons which become an important source of the pions observed in the final state.

In a baryonic environment pions are reabsorbed with a certain probability via the sequential process $\pi \mathrm{N} \rightarrow \Delta$ and $\Delta \mathrm{N} \rightarrow \mathrm{NN}$. Experiments on "true pion absorption" found a cross section for this process of about 1 barn for pions with kinetic energies of 125 and $187 \mathrm{MeV}$ impinging on heavy nuclei [65, 66. Such an absorption cross section corresponds to a mean free path of about $5 \mathrm{fm}$ at normal nuclear matter density. The mean free path decreases with increasing baryon density. Hence pion reabsorption is enhanced at SIS energies as compared to SPS energies and also in heavy collision systems as compared to light ones. In the framework of transport models, pion production, propagation and reabsorption depends sensitively on the in-medium properties of the $\Delta$ resonance (i.e. lifetime, spectral function, interaction cross sections) which are not well known. This might be the reason that transport models still have difficulties to reproduce the pion yields and momentum distributions measured in $\mathrm{A}+\mathrm{A}$ collisions [64].

Due to their large cross section, most of the pions are trapped in the dense reaction zone and have a chance to leave only when the hadrons cease to interact. Therefore, the measured pion phase space distributions mainly reflect the fireball properties at freeze-out. However, at low beam energies where the pion to nucleon ratio is small, high-energy pions freeze-out much earlier as they are beyond the kinematical region of the $\Delta$ resonance. These pions offer the possibility to study the dense phase of the collision and therefore are important messengers of the reaction dynamics.

In this chapter we review the data on pion multiplicites, their transverse momentum and angular distributions (rapidity distributions have already been discussed in chapter 2). Moreover, we discuss data on the correlation between pion emission and the reaction plane which provide indications for a time scale in pion emission. Finally we present data on the pion isospin ratios which can give access to the size of the pion emitting source [67.

\subsection{Multiplicities}

Early experiments performed with the streamer chamber at the LBL found that the $\pi^{-}$multiplicity per event increases linearly with the number of participants. In $\mathrm{Ar}+\mathrm{KCl}$ collisions the ratio of $\pi^{-}$mesons to participating protons is about 0.08 for $1 \mathrm{AGeV}$ beam energy and about 0.2 for $1.8 \mathrm{AGeV}$ [68]. 
Very similar results were obtained for La+La collisions [69]. Taking into account the isospin asymmetry of the La-nucleus, the total pion multiplicity $\mathrm{n}_{\pi}$ was calculated from the measured $\pi^{-}$multiplicity. The number of participating nucleons $\mathrm{A}_{\text {part }}$ was determined from the charge sum of projectile spectator fragments measured with a plastic scintillator hodoscope at forward angles. It was found, that for La+La collisions the ratio of the total pion multiplicity to the number of participant nucleons varies from 0.035 at a beam energy of $0.53 \mathrm{AGeV}$ to about 0.18 at $1.35 \mathrm{AGeV}$. At a beam energy of $1 \mathrm{AGeV}$, for example, both the $\mathrm{Ar}+\mathrm{KCl}$ and the $\mathrm{La}+\mathrm{La}$ measurements yield a ratio of $\mathrm{n}_{\pi} / \mathrm{A}_{\text {part }} \approx 0.12$.

Table 3.1: Total pion multiplicity per number of participating nucleons for $\mathrm{A}+\mathrm{A}$ collisions at $1 \mathrm{AGeV}$

\begin{tabular}{|c|c|c|c|c|}
\hline $\mathrm{A}+\mathrm{A}$ & $\mathrm{n}_{\pi} / \mathrm{A}_{\text {part }}$ & $\pi$ acceptance & measured data & Ref. \\
\hline $\mathrm{C}+\mathrm{C}$ & $0.168 \pm 0.012$ & midrapidity & $\pi^{0}$ & {$[53$} \\
\hline $\mathrm{Ar}+\mathrm{Ca}$ & $0.09 \pm 0.009$ & midrapidity & $\pi^{0}$ & {$[70,72]$} \\
\hline $\mathrm{Ar}+\mathrm{KCl}$ & $0.114 \pm 0.015$ & $4 \pi$ & $\pi^{-}$ & {$[68]$} \\
\hline $\mathrm{Ni}+\mathrm{Ni}$ & $0.12 \pm 0.01$ & $40^{\circ}<\Theta_{l a b}<48^{\circ}$ & $\pi^{+}, \pi^{-}$ & {$[73$} \\
\hline $\mathrm{Ni}+\mathrm{Ni}$ & $0.13 .62 \pm 0.139$ & $32^{\circ}<\Theta_{\text {lab }}<150^{\circ}$ & $\pi^{+}, \pi^{-}$ & {$[22]$} \\
\hline $\mathrm{Kr}+\mathrm{Zr}$ & $0.075 \pm 0.021$ & midrapidity & $\pi^{0}$ & {$[70,72]$} \\
\hline $\mathrm{La}+\mathrm{La}$ & $0.119 \pm 0.013$ & $4 \pi$ & $\pi^{-}$ & {$[69]$} \\
\hline $\mathrm{Au}+\mathrm{Au}$ & $0.069 \pm 0.015$ & midrapidity & $\pi^{\circ}$ & {$[72,13]$} \\
\hline $\mathrm{Au}+\mathrm{Au}$ & $0.074 \pm 0.012$ & $30^{\circ}<\Theta_{l a b}<150^{\circ}$ & $\pi^{+}, \pi^{-}$ & {$[20]$} \\
\hline $\mathrm{Au}+\mathrm{Au}$ & $0.09 \pm 0.01$ & $40^{\circ}<\Theta_{l a b}<48^{\circ}$ & $\pi^{+}, \pi^{-}$ & {$[71]$} \\
\hline
\end{tabular}

Table 3.1 presents a compilation of average pion multiplicities $\mathrm{n}_{\pi}$ as a function of $\mathrm{A}_{\text {part }}$ for $\mathrm{A}+\mathrm{A}$ collisions at $1 \mathrm{AGeV}$. The table includes data from $\mathrm{Au}+\mathrm{Au}$ collisions measured recently by three different experiments at SIS [71, 20, 72]. The KaoS [71] and TAPS [72] data were taken around midrapidity and extrapolated to $4 \pi$ assuming isotropic pion emission in the fireball frame. The FOPI data 20] were measured within $30^{\circ}<\Theta_{l a b}<150^{\circ}$ and are somewhat lower than the KaoS data for central $\mathrm{Au}+\mathrm{Au}$ collisions. The differences in the data might result from different methods in the determination of $\mathrm{A}_{\text {part }}$ (the inclusive differential cross-sections as a function of transverse momentum as measured by the two experiments agree within $10 \%$, see Fig. 3.2).

The pion-to-participant ratios in Table 3.1 are not constant when changing the mass of the collision system. There seems to be a trend of decreasing $\mathrm{n}_{\pi} / \mathrm{A}_{\text {part }}$ values with increasing $\mathrm{A}$ : the largest value is found in $\mathrm{C}+\mathrm{C}$, the smallest in $\mathrm{Au}+\mathrm{Au}$ collisons whereas in the mass range from $\mathrm{Ar}+\mathrm{KCl}$ to $\mathrm{La}+\mathrm{La}$ the values stay constant. It is interesting to note that the corresponding value for nucleon-nucleon collisions at $1 \mathrm{GeV}$ beam energy is even larger than the one for $\mathrm{C}+\mathrm{C}$ (see Table 3.2).

In Table 3.2 and Fig. 3.1 we address the question how the pion multiplicity per participant evolves with beam energy. The findings from symmetric nuclear systems are compared with the corresponding numbers from nucleon-nucleon collisions as compiled in reference |74. Following the line of arguments presented in Chapter 2 we have rescaled the values of the number of pions per nucleon from reference 74 ] by the ratio $\sigma_{\text {inel }} / \sigma_{\text {tot }}$ for a consistent comparison between $\mathrm{N}+\mathrm{N}$ and $\mathrm{A}+\mathrm{A}$ collisions.

For $\mathrm{A}+\mathrm{A}$ collisions the values of $\mathrm{n}_{\pi} / \mathrm{A}_{\text {part }}$ increase from $\mathrm{n}_{\pi} / \mathrm{A}_{\text {part }}=0.1-0.2$ at LBL/SIS energies to $\approx 0.7$ at Dubna energies and to about 1 for AGS energies. At SPS energies, the pion-to-participant ratio reaches a value of $\mathrm{n}_{\pi} / \mathrm{A}_{\text {part }} \approx 5$ which means that a new "state" of meson dominated matter has been created.

The pion-to-participant ratio measured in $\mathrm{N}+\mathrm{N}$ collisions clearly deviates from the $\mathrm{A}+\mathrm{A}$ data for low and high beam energies. At BEVALAC/SIS energies, the ratio $\mathrm{n}_{\pi} / \mathrm{A}_{\text {part }}$ for $\mathrm{A}+\mathrm{A}$ collisions is in average a factor of about 2 smaller than for $\mathrm{N}+\mathrm{N}$ collisions. This difference increases with increasing A. At SPS energies, $\mathrm{n}_{\pi} / \mathrm{A}_{\text {part }}$ is about $35 \%$ larger in $\mathrm{A}+\mathrm{A}$ than in $\mathrm{N}+\mathrm{N}$ interactions. The discrepancy at SIS energies may be due to true pion absorption which proceeds via sequential processes occuring in $\mathrm{A}+\mathrm{A}$ collisions $(\pi \mathrm{N} \rightarrow \Delta$ and $\Delta \mathrm{N} \rightarrow \mathrm{NN})$. Pion reabsorption requires high nucleon densities i.e. a small value of $\mathrm{n}_{\pi} / \mathrm{A}_{\text {part }}$ which is realized at low beam energies. At SPS energies, multiple pion production is possible and pion reabsorption is suppressed because of the low baryon density. 


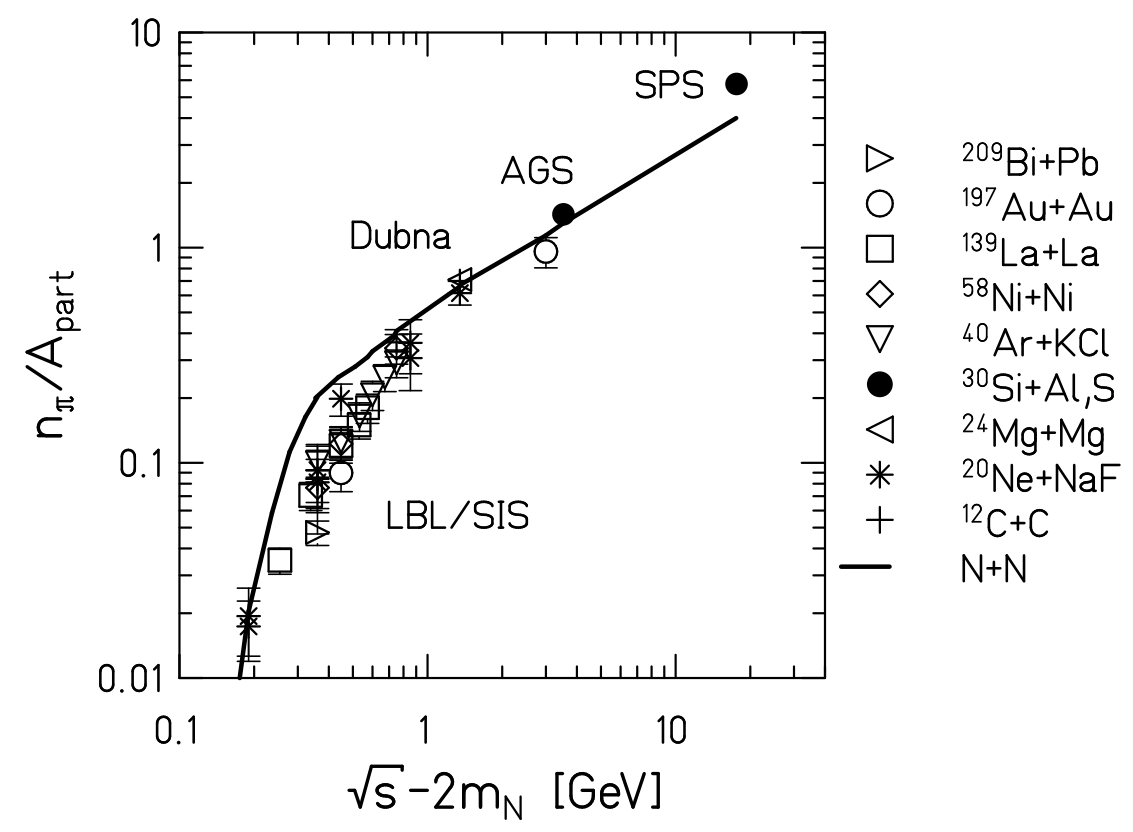

Figure 3.1: Compilation of the pion multiplicity per participating nucleon as a function of the kinetic energy of two colliding nucleons in the center-of-mass frame. The A+A data are taken from 74, 82, 77, 79, 68, 69, 53. The $\mathrm{N}+\mathrm{N}$ data (solid line) are taken from [74 but rescaled by $\sigma_{\text {inel }} / \sigma_{\text {tot }}$ (see text).

Table 3.2: Total pion multiplicity per participating nucleon for different beam energies. The value for $\mathrm{Au}+\mathrm{Au}$ at $1 \mathrm{AGeV}$ is averaged over the experimental results listed in Table 3.1.

\begin{tabular}{|c|c|c|c|c|c|}
\hline $\mathrm{A}_{1}+\mathrm{A}_{2}$ & $\overline{\mathrm{E}_{\text {beam }}(\mathrm{AGeV})}$ & $\sqrt{s_{N N}}-2 m_{N}(\mathrm{GeV})$ & $\mathrm{n}_{\pi} / \mathrm{A}_{\text {part }}$ & Ref. & \\
\hline $\mathrm{La}+\mathrm{La}$ & 0.53 & 0.25 & $0.035 \pm 0.005$ & 69 & \\
\hline $\mathrm{N}+\mathrm{N}$ & 1.0 & 0.45 & $0.24 \pm 0.008$ & 74 & \\
\hline $\mathrm{La}+\mathrm{La}$ & & & $0.12 \pm 0.017$ & 69 & \\
\hline $\mathrm{Au}+\mathrm{Au}$ & & & $0.086 \pm 0.1$ & 20,71 , & 72 \\
\hline $\mathrm{N}+\mathrm{N}$ & 1.8 & 0.75 & $0.41 \pm 0.01$ & 74 & \\
\hline $\mathrm{Ar}+\mathrm{KCl}$ & & & $0.18 \pm 0.03$ & 68 & \\
\hline $\mathrm{N}+\mathrm{N}$ & 3.66 & 1.35 & $0.67 \pm 0.08$ & 74 & \\
\hline $\mathrm{Mg}+\mathrm{Mg}$ & & & $0.7 \pm 0.04$ & 74 & \\
\hline $\mathrm{N}+\mathrm{N}$ & 10.7 & 3.0 & $1.16 \pm 0.1$ & 74 & \\
\hline $\mathrm{Si}+\mathrm{Al}$ & 13.7 & 3.5 & $1.4 \pm 0.14$ & 74 & \\
\hline $\mathrm{Au}+\mathrm{Au}$ & 10.7 & 3.0 & $1.11 \pm 0.18$ & 74 & \\
\hline $\mathrm{N}+\mathrm{N}$ & 200 & 17.6 & $4.0 \pm 0.1$ & $\mid 74$ & \\
\hline $\mathrm{S}+\mathrm{S}$ & 200 & 17.6 & $5.4 \pm 0.2$ & 74 & \\
\hline $\mathrm{Pb}+\mathrm{Pb}$ & 158 & 15.4 & $5.1 \pm 0.2$ & 57 & \\
\hline
\end{tabular}


Table 3.3: Pion inverse slope parameters from a fit to the spectra according to $\mathrm{d}^{3} \sigma / \mathrm{dp}^{3}=\mathrm{C}_{1} \exp \left(-\mathrm{E} / \mathrm{T}_{1}\right)$ $+\mathrm{C}_{2} \exp \left(-\mathrm{E} / \mathrm{T}_{2}\right)$ The data were taken at SIS energies.

\begin{tabular}{|c|c|c|c|c|c|}
\hline $\mathrm{A}+\mathrm{A}$ & $\mathrm{E}[\mathrm{AGeV}$ & $\pi$ & $\mathrm{T}_{1}[\mathrm{MeV}]$ & $\mathrm{T}_{2}[\mathrm{MeV}]$ & Ref. \\
\hline$\overline{\mathrm{Au}+\mathrm{Au}}$ & 1.0 & $\pi^{+}$ & $45 \pm 3$ & $76 \pm 3$ & 777 \\
\hline $\mathrm{Au}+\mathrm{Au}$ & 1.0 & $\pi^{+}$ & $49 \pm 2$ & $85 \pm 3$ & |71] \\
\hline $\mathrm{Au}+\mathrm{Au}$ & 1.0 & $\pi$ & $41 \pm 3$ & $76 \pm 3$ & |71| \\
\hline $\mathrm{Au}+\mathrm{Au}$ & 1.0 & $\pi^{+}$ & $49 \pm 4$ & $96 \pm 10$ & [20] \\
\hline $\mathrm{Au}+\mathrm{Au}$ & 1.0 & $\bar{\pi}$ & $42 \pm 3$ & $96 \pm 10$ & (20) \\
\hline $\mathrm{Au}+\mathrm{Au}$ & 1.0 & $\pi^{o}$ & $38 \pm 4$ & $\overline{78 \pm 4}$ & 72 \\
\hline $\mathrm{Ni}+\mathrm{Ni}$ & $\overline{1.0}$ & $\pi^{+}$ & $45 \pm 3$ & $\overline{75 \pm 3}$ & 77 \\
\hline $\mathrm{Ni}+\mathrm{Ni}$ & 1.0 & $\pi^{+}$ & $47 \pm 3$ & $\overline{77 \pm 3}$ & 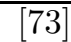 \\
\hline $\mathrm{Ni}+\mathrm{Ni}$ & 1.06 & $\pi^{+}$ & $49.4 \pm 3.7$ & $96.4 \pm 5.1$ & 22 \\
\hline $\mathrm{Ni}+\mathrm{Ni}$ & 1.06 & $\pi^{-}$ & $42.2 \pm 2.7$ & $96.4 \pm 5.1$ & 22 \\
\hline $\mathrm{Kr}+\mathrm{Zr}$ & 1.0 & $\pi^{o}$ & $48 \pm 8$ & $76 \pm 10$ & 72 \\
\hline $\mathrm{Ne}+\mathrm{NaF}$ & 1.0 & $\pi^{+}$ & $43 \pm 3$ & $63 \pm 3$ & (77) \\
\hline $\mathrm{Ni}+\mathrm{Ni}$ & 0.8 & $\pi^{+}$ & $40 \pm 3$ & $68 \pm 3$ & $\mid 77$ \\
\hline $\mathrm{Ni}+\mathrm{Ni}$ & 1.45 & $\pi^{+}$ & $54.3 \pm 3.3$ & $103.8 \pm 4.9$ & 22 \\
\hline $\mathrm{Ni}+\mathrm{Ni}$ & 1.8 & $\pi^{+}$ & $50 \pm 3$ & $95 \pm 3$ & [77] \\
\hline $\mathrm{Ni}+\mathrm{Ni}$ & 1.8 & $\pi^{+}$ & $36 \pm 3$ & $90 \pm 3$ & |73] \\
\hline $\mathrm{Ni}+\mathrm{Ni}$ & 1.8 & $\pi^{-}$ & $40 \pm 3$ & $91 \pm 3$ & |73| \\
\hline $\mathrm{Ni}+\mathrm{Ni}$ & 1.93 & $\pi^{+}$ & $56.6 \pm 4.4$ & $111.7 \pm 9.5$ & 22 \\
\hline $\mathrm{Ar}+\mathrm{KCl}$ & 1.8 & $\pi^{-}$ & 59 & 102 & 76 \\
\hline $\mathrm{C}+\mathrm{C}$ & 0.8 & $\pi^{o}$ & $50 \pm 4$ & - & 53 \\
\hline $\mathrm{C}+\mathrm{C}$ & 1.0 & $\pi^{o}$ & $54 \pm 3$ & - & 53 \\
\hline $\mathrm{C}+\mathrm{C}$ & 2.0 & $\pi^{o}$ & $83 \pm 2$ & - & 53 \\
\hline $\mathrm{C}+\mathrm{C}$ & 1.0 & $\pi^{+}, \pi^{-}$ & $45 \pm 3$ & $62 \pm 3$ & 83 \\
\hline $\mathrm{C}+\mathrm{C}$ & $\overline{2.0}$ & $\pi^{+}, \pi^{-}$ & $40 \pm 3$ & $86 \pm 3$ & 83 \\
\hline
\end{tabular}

\subsection{Transverse momentum distributions}

Pioneering experiments at the LBL BEVALAC found that the transverse momentum spectra of negatively charged pions measured in central $\mathrm{Ar}+\mathrm{KCl}$ collisions at $1.8 \mathrm{AGeV}$ exhibit a nearly exponential shape with a characteristic enhancement at low transverse momenta [76]. Recent experiments performed at SIS/GSI have extended the study of charged and neutral pion production to very heavy collision systems and found similar effects. Fig. 3.2 shows the pion invariant cross sections versus transverse momentum for $\mathrm{Au}+\mathrm{Au}$ at $1 \mathrm{AGeV}$ measured around midrapidity by three different setups. The pion spectra deviate from a single exponential and have been parameterized by a superposition of two Maxwell-Boltzmann distributions:

$$
d^{3} \sigma / d p^{3}=C_{1} \exp \left(-E / T_{1}\right)+C_{2} \exp \left(-E / T_{2}\right)
$$

Table 3.3 presents a compilation of the inverse slope parameters as obtained by a fit to the measured spectra according to equ. 3.1. The values of the inverse slope parameters $T_{1}$ and $T_{2}$ are correlated and depend somewhat on the measured momentum range. Nevertheless, the fit results have some features in common: $\mathrm{T}_{1}$ has values between 40 and $50 \mathrm{MeV}$ for most of the systems and $\mathrm{T}_{2}$ increases with both the mass number of the colliding nuclei and the bombarding energy. For $\mathrm{Au}+\mathrm{Au}$ collisions at $1 \mathrm{AGeV}$ the pion inverse slope parameters are found to be constant for different center-of-mass polar emission angles [20]. Any interpretation of the slope parameters in Table 3.3 should take into account the correlations between the different fit parameters. What would be needed in addition to the numbers presented in Table 3.3 are the cross correlations between the two slope factors and relative yields in the two components. 


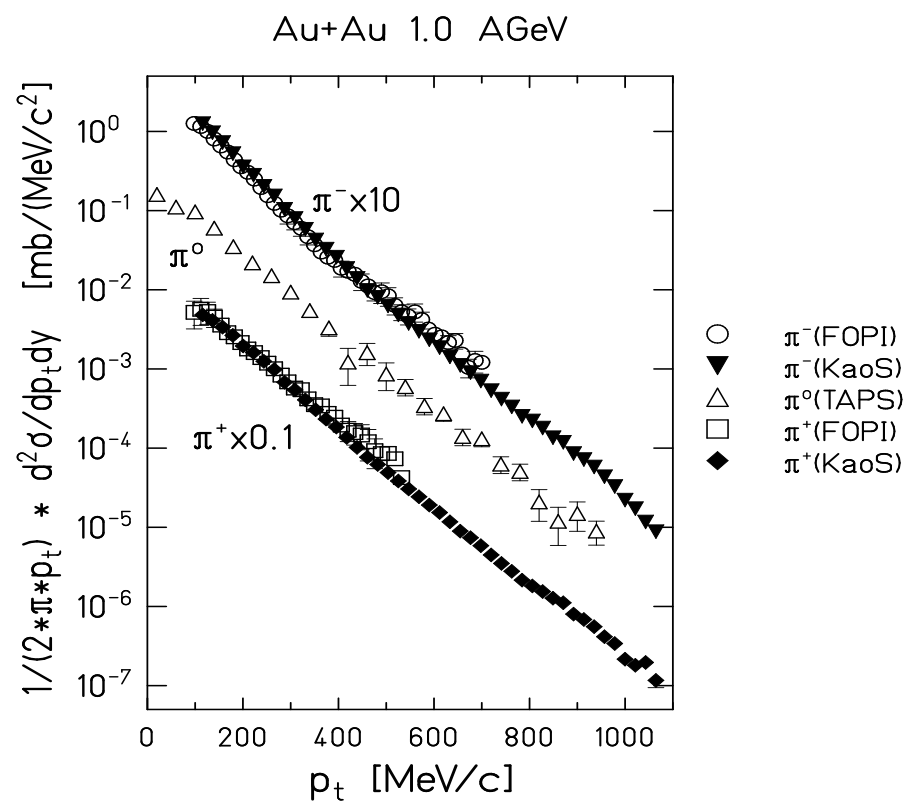

Figure 3.2: Invariant pion production cross sections as a function of transverse momentum for $\mathrm{Au}+\mathrm{Au}$ at 1 AGeV measured around midrapidity by FOPI $\left(\pi^{-}\right.$: open circles, $\pi^{+}$: open squares [20], KaoS (full symbols [71]) and TAPS (open triangles [72]).

The enhancement at low pion momenta (described by the $\mathrm{T}_{1}$ component) was explained by the contribution of pions from delta resonance decay [76]. In this case the pion transverse momentum is strongly influenced by the Q-value of the decay and does not reflect the "temperature" of the nuclear fireball. This interpretation of the pion spectra was motivated by the fact that the pion production cross section in proton-proton collisions up to about $2 \mathrm{GeV}$ bombarding energy is dominated by the $\Delta$ resonance [61, 62]. Recent data on pion production in $\mathrm{Au}+\mathrm{Au}$ collisions at $1 \mathrm{AGeV}[77]$ and $\mathrm{Ni}+\mathrm{Ni}$ collisions at 1.06, 1.45 and $1.93 \mathrm{AGeV}$ [17] were analyzed in terms of thermal models including pions from baryonic resonances which are embedded in a radial flow. In Ref. 77 it was assumed that all pions stem from resonance decays and the measured pion momentum distribution could be reproduced by varying the in-medium spectral function of the resonances. In the analysis of Ref. [17], the pion transverse momentum spectra were explained by a (high-energy) thermal component and a contribution from $\Delta$ decays at low transverse momenta. In this approach, the $\Delta$ mass distribution is parameterized by a relativistic Breit-Wigner form with the maximum fixed to the free mass of $1232 \mathrm{MeV}$ and a momentum dependent width. When increasing the beam energy from 1.06 to $1.93 \mathrm{AGeV}$ the ratio $\Delta$ /baryons at freeze-out increases from $10 \%$ to $18 \%$ whereas the ratio of pions from $\Delta$ decays to the total pion multiplicity decreases from $77 \%$ to $66 \%$ [17].

In a recent invariant mass analysis of $\mathrm{p} \pi^{ \pm}$pairs in $\mathrm{Ni}+\mathrm{Cu}$ collisions at $1.97 \mathrm{AGeV}$ correlations from the decays of the $\Delta$ resonance have been observed $\left[78\right.$. It was found, that the maxima of the $\Delta^{++}$and $\Delta^{0}$ mass distributions are shifted to lower values as compared to the free spectral function. This mass reduction is maximized in central collisions and has been interpreted as a simple kinematic phase space effect.

It is still under debate, whether the high-energy component of the pion spectra also reflects resonance decay kinematics [20, 64, 80] or represents the thermal pions 81. Fig. 3.3 shows the inverse slope parameter of the high-energy $\left(\mathrm{T}_{2}: \mathrm{E}_{c m}^{\mathrm{knn}}>300 \mathrm{MeV}\right)$ pions for $\mathrm{Ni}+\mathrm{Ni}$ and $\mathrm{Au}+\mathrm{Au}$ collisions at $1 \mathrm{AGeV}$ as a function of the number of participants. The inverse slope parameter of the high-energy pions increases with increasing of $\mathrm{A}_{\text {part }}$ from $\mathrm{T}_{2}=60 \mathrm{MeV}$ to $80 \mathrm{MeV}$ (for $\mathrm{A}_{\text {part }}=300$ ). Furthermore, its value measured in central $\mathrm{Ni}+\mathrm{Ni}$ collisions agrees with the one from peripheral $\mathrm{Au}+\mathrm{Au}$ collisions. These findings show that the pion spectral slope at high $\mathrm{p}_{T}$ depends on the size of the fireball and is not influenced by the comoving spectator 


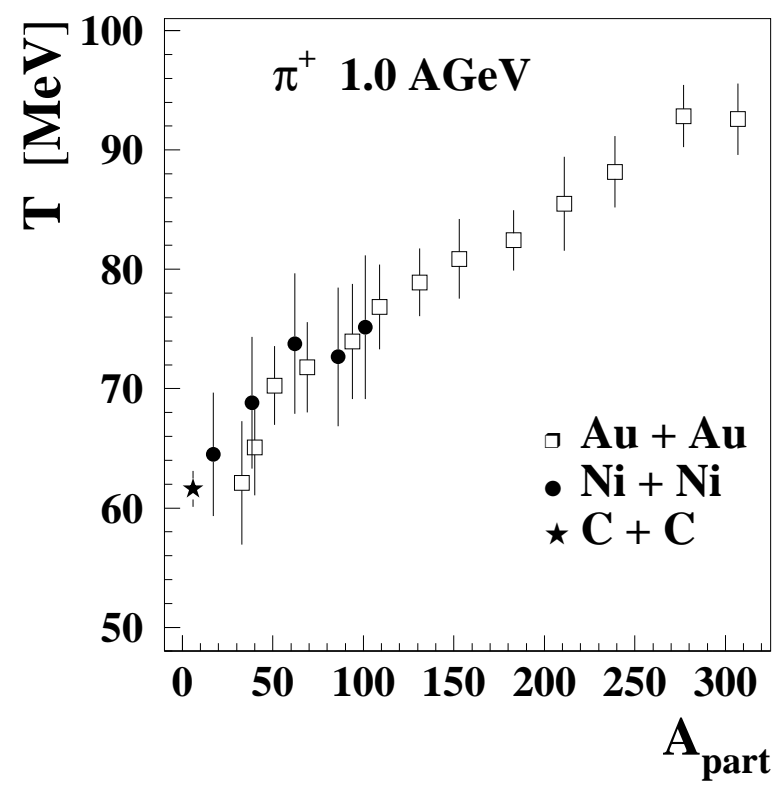

Figure 3.3: Inverse slope parameter of the high-energy pions $\left(\mathrm{E}_{c m}^{k i n}>300 \mathrm{MeV}\right)$ for $\mathrm{Ni}+\mathrm{Ni}$ and $\mathrm{Au}+\mathrm{Au}$ collisions at $1 \mathrm{AGeV}$ as a function of the number of participant nucleons [73, 83].

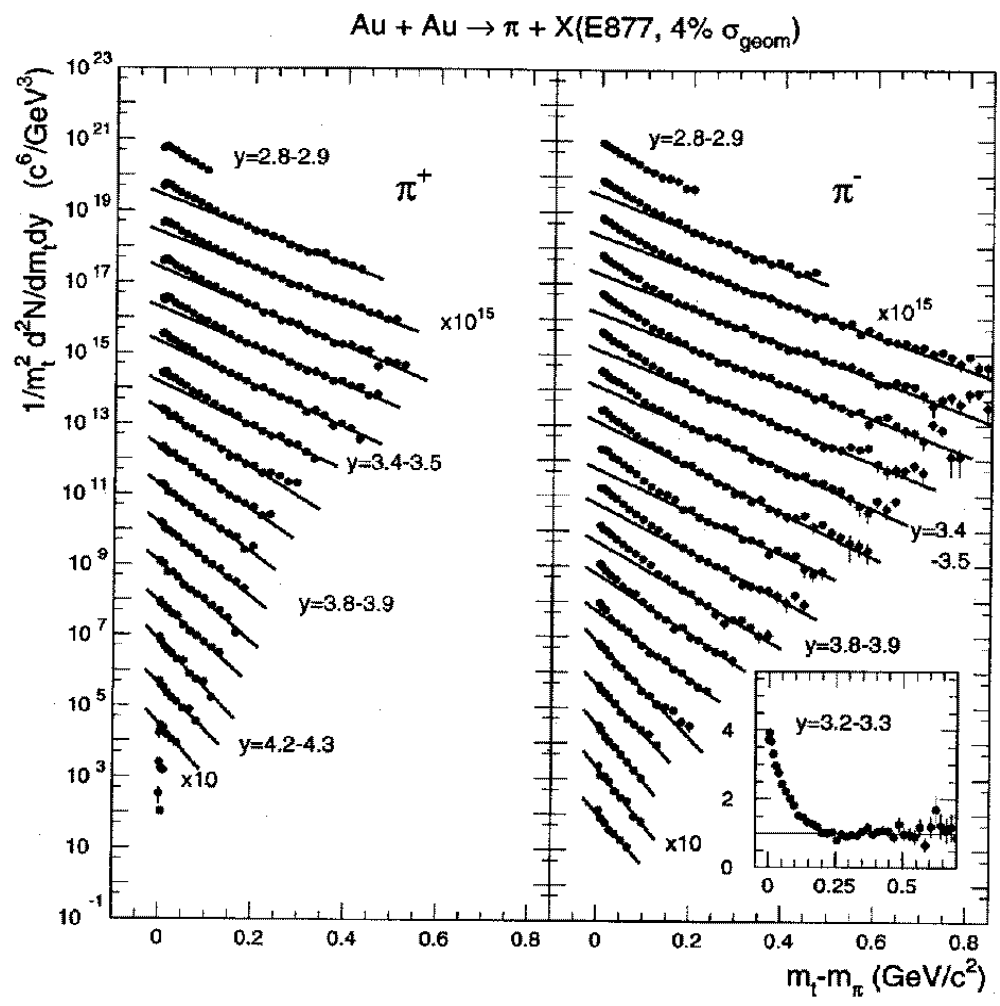

Figure 3.4: Transverse mass distributions of $\pi^{+}$and $\pi^{-}$mesons measured at forward rapidities in central $\mathrm{Au}+\mathrm{Au}$ collisions at $10 \mathrm{AGeV}$ beam energy 26. 


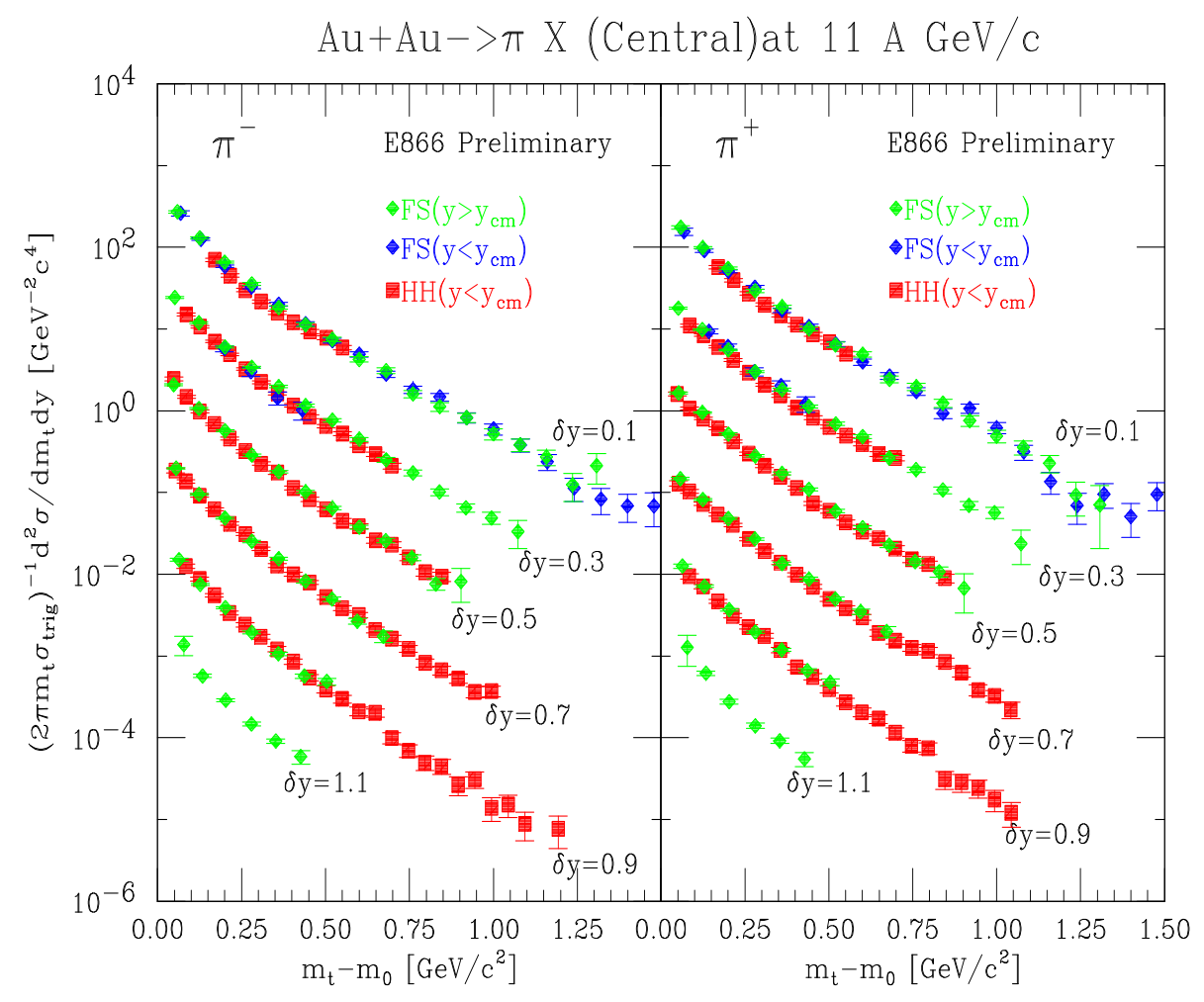

Figure 3.5: Transverse mass distributions of $\pi^{+}$and $\pi^{-}$mesons measured around midrapidity in central $\mathrm{Au}+\mathrm{Au}$ collisions at $10 \mathrm{AGeV}$ beam energy [24].

fragments. It should be noted that the data shown in Fig. 3.3 are derived from pion spectra which are integrated over the azimuthal angle.

The high-energy pion yield was studied as a function of collision centrality for heavy collision systems at SIS energies [77, 82]. In contrast to low-energy pions (which dominate the pion yield) the multiplicity of high-energy pions $\left(\mathrm{E}_{c . m}^{t o t} .>671 \mathrm{MeV}\right)$ increases more than linearly with the number of participating nucleons (the total pion c.m.energy of $671 \mathrm{MeV}$ corresponds to the threshold for $\mathrm{K}^{+}$production in a $\mathrm{NN}$ collision): assuming $\mathrm{M} \propto \mathrm{A}_{\text {part }}^{\alpha}$, values of $\alpha=1.86 \pm 0.19$ and $1.63 \pm 0.19$ were determined for $\mathrm{Bi}+\mathrm{Pb}$ collisions at $0.8 \mathrm{AGeV}$ and $\mathrm{Au}+\mathrm{Au}$ at $1 \mathrm{AGeV}$, respectively. The production of these high-energy pions is "subthreshold" and therefore requires collective effects like multiple baryon-baryon interactions. As these processes are strongly enhanced in the dense medium, high-energy pions seem to probe the high-density stage of the collision. This picture is supported by the pion azimuthal emission pattern and the $\pi^{+} / \pi^{-}$ ratio as discussed in the next sections.

The "low- $\mathrm{p}_{T}$ enhancement" of the pion yield is also found at higher bombarding energies. Fig. 3.4 shows $\pi^{+}$and $\pi^{-}$transverse mass distributions measured at forward rapidities in central $\mathrm{Au}+\mathrm{Au}$ collisions at $10 \mathrm{AGeV}$ beam energy [26]. At low transverse masses a clear enhancement above the pure exponential is visible. The insert in Fig. 3.4 shows the ratio of the pion yield to an exponential (fitted to the spectra above $\mathrm{m}_{T}-\mathrm{m}_{\pi}>0.2 \mathrm{GeV} / \mathrm{c}$ ) for a given rapidity bin. The effect is most pronounced at midrapidity (see also Fig. 3.5, 24]). The low- $\mathrm{p}_{T}$ enhancement of the pions was again explained by the contribution of pions from $\Delta$ decays $[25$.

This effect is also clearly visible in $\mathrm{Pb}+\mathrm{Pb}$ collisions at SPS energies (see Fig. 2.9, [44]). However, the large pion multiplicity per participant of $\mathrm{n}_{\pi} / \mathrm{A}_{\text {part }} \approx 5$ (see Table 3.2 and Fig. 3.1) indicates that the pion yield at low $\mathrm{p}_{T}$ is due to a new source of pions other than the decay of baryonic resonances. Relativistic transport calculations consider string fragmentation and $\rho$ and $\omega$ decays to be the dominant source of low $\mathrm{p}_{T}$ pions. 


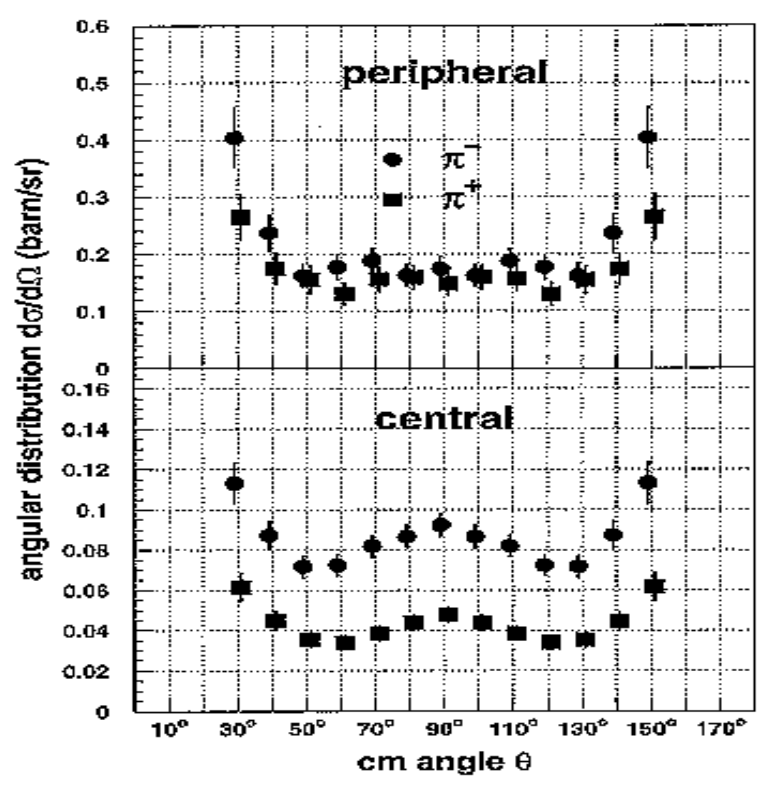

Figure 3.6: Pion angular distributions from peripheral (top) and central (bottom) $\mathrm{Au}+\mathrm{Au}$ collisions at $1.06 \mathrm{AGeV}$ [20].

Earlier studies of light projectiles on heavy targets revealed a stronger pion enhancement near target rapidity [41, 84]. It remains to be seen whether this trend persists also for the heavy $\mathrm{Pb}$ projectile and what differences are found between central and semi-central collisions.

\subsection{Angular distributions}

Pions produced in proton-proton collisions exhibit a strong forward-backward peaked angular distribution which is a consequence of the dominant p-wave production channel [85]. Pions emitted from a thermalized fireball formed in nucleus-nucleus collisions, however, should exhibit an isotropic angular distribution in the center-of-mass system. It was found in $\mathrm{Ne}+\mathrm{NaF}$ and $\mathrm{Ar}+\mathrm{KCl}$ collisions that the pion emission pattern still contains a forward-backward peaked contribution [85, 79. The anisotropy is larger for peripheral than for central collisions. For central $\mathrm{Ar}+\mathrm{KCl}$ collisions at $1.8 \mathrm{AGeV}$, for example, about $90 \%$ of the pions are emitted isotropically 11. This effect is caused by pion reabsorption and reemission which occurs more freqently in central events [64] and can be also considered to be a higher degree of thermalization.

Fig. 3.6 presents recent results of the FOPI Collaboration which measured the pion polar angular distribution in $\mathrm{Au}+\mathrm{Au}$ collisions at $1 \mathrm{AGeV}$ [20]. A forward-backward enhancement is clearly visible both for peripheral and central collisions. In central collisions, however, an additional structure appears at $\Theta_{c m}$ $=90^{\circ}$ : the emission of $\pi^{+}$and $\pi^{-}$is enhanced at midrapidity. This effect is also observed for 'minimum bias' collisions if pion kinetic energies above $365 \mathrm{MeV}$ are selected [20].

Experiments at LBL/SIS have established the azimuthally anisotropic emission of particles from the reaction zone of two colliding nuclei: the directed flow of nucleons in the reaction plane ("side splash") and the preferred emission of nucleons perpendicular to the reaction plane ("squeeze-out") 86, 87, 88, 89. 90, 91, 92]. A collective out-of-plane emission was predicted by early hydrodynamical calculations and interpreted as a dynamical squeeze out of matter due to the build-up of pressure in the interaction zone [2].

First measurements of the emission pattern of pions in symmetric as well in asymmetric heavy-ion collisions were done with the streamer chamber at the LBL BEVALAC [93, 94]. These studies showed evidence for a weak in-plane correlation in the $\pi^{-}$emission pattern. Similar findings for asymmetric systems were reported by the DIOGENE group 95]. These measurements indicated a preferential in-plane emission of charged pions on the projectile side. This behaviour was attributed to a stronger pion absorption by the heavier target spectator remnant on the side opposite to the projectile. 


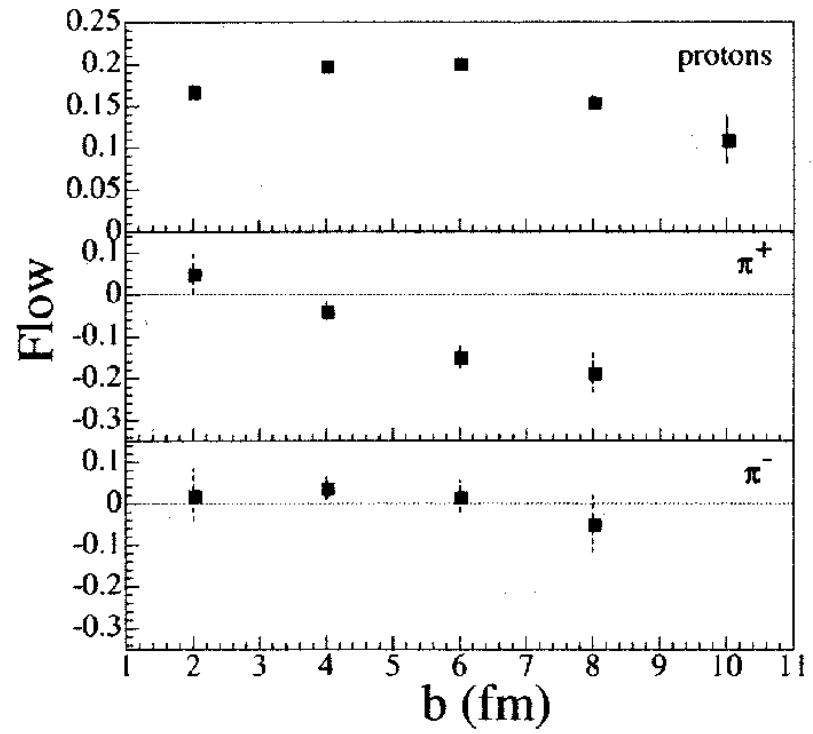

Figure 3.7: Sidewards flow as a function of impact parameter for protons (top), $\pi^{+}$(middle) and $\pi^{-}$(bottom) in $\mathrm{Au}+\mathrm{Au}$ collisions at $1.15 \mathrm{AGeV}$ [96].

Recently, the directed in-plane flow of pions and protons was measured in $\mathrm{Au}+\mathrm{Au}$ collsions at 1.15 $\mathrm{AGeV}$ as a function of impact parameter by the EOS collaboration [96]. They found that the average in-plane transverse velocity $\left\langle p_{x} / m>\right.$ of pions and protons exhibits a $\mathrm{S}$ shape as a function of rapidity. The magnitude of the flow is defined as the slope of the $\mathrm{S}$ curve at midrapidity. This slope is shown in Fig. 3.7 for protons, $\pi^{+}$and $\pi^{-}$as a function of impact parameter. The protons clearly show a flow signal for all impact parameters. The positively charged pions show little flow for central collisions and a large antiflow for peripheral collisions. The negatively charged pions do not exhibit a clear trend.

Transport calculations predicted a weak pionic flow in central collisions due to the flow of baryon resonances from which they are produced but an antiflow behaviour in peripheral collisions as a result of the shadowing by spectators 63, 97]. A weak pionic antiflow was also found in central collisions of $\mathrm{Au}+\mathrm{Au}$ at $10.8 \mathrm{AGeV}$ both for $\pi^{+}$and $\pi^{-}$[26] and explained in a similar way 98 .

Very recently a measurement of pionic flow was performed in $\mathrm{Au}+\mathrm{Au}$ collisions at $1 \mathrm{AGeV}$ as a function of the impact parameter, rapidity and pion energy by the KaoS Collaboration [71. In peripheral collisions, a directed in-plane antiflow was observed for low $\mathrm{p}_{T}$ pions in accordance with the $\pi^{+}$data of the EOS Collaboration (their integrated pion yields are dominated by low-energy pions). For high $\mathrm{p}_{T}$ pions, however, a (positive) flow behaviour was measured. This is demonstrated in Fig. 3.8 which shows the spectral ratio of pions emitted into the event plane to the "projectile side" over the ones emitted to the "target side" $\left(\mathrm{R}=\mathrm{N}_{p} / \mathrm{N}_{t}\right)$ for peripheral $\mathrm{Au}+\mathrm{Au}$ collisions at $1 \mathrm{AGeV}$. As this measurement is performed at target rapidity $\left(0.01<\mathrm{y} / \mathrm{y}_{\text {beam }}<0.1\right)$ a value of $\mathrm{N}_{p} / \mathrm{N}_{t}$ smaller (larger) than unity means flow (antiflow).

The data presented in Fig. 3.8 show that the flow behaviour of negative and positive pions is similar. This is in contradiction to the data presented in Fig. 3.7 where the positively charged pions exhibit a clear antiflow signal at $b=8 \mathrm{fm}$ whereas the antiflow of the negatively charged pions is consistent with zero.

The KaoS data (Fig. 3.8) can be explained by the shadowing of high $\mathrm{p}_{T}$ pions by the spectator fragments in the early phase of the collision. This effect produces (i) a pronounced "squeeze-out" signal (see below) and (ii) a directed in-plane flow. The low $\mathrm{p}_{T}$ pions, however, freeze out at a later time and the rescattering at the spectator fragments produces an antiflow signal. Fig. 3.9 sketches the matter configuration at 4, 8 and $20 \mathrm{fm} / \mathrm{c}$ after the first touch of the nuclei according to a QMD calculation for $\mathrm{Au}+\mathrm{Au}$ at $1 \mathrm{AGeV}$ and at an impact parameter of $b=7 \mathrm{fm}$ [99. The "snapshots" exemplify the effect of pion shadowing by spectator matter at different stages of the collision. QMD calculations predict different freeze-out times and densities for low and high energy pions. The model calculations find a correlation between high-energy pions, early freeze-out times and high freeze-out densities [80]. This scenario is supported by the data shown in Fig. 3.8 which demonstrate for the first time experimentally that pion emission is correlated to the reaction dynamics. 


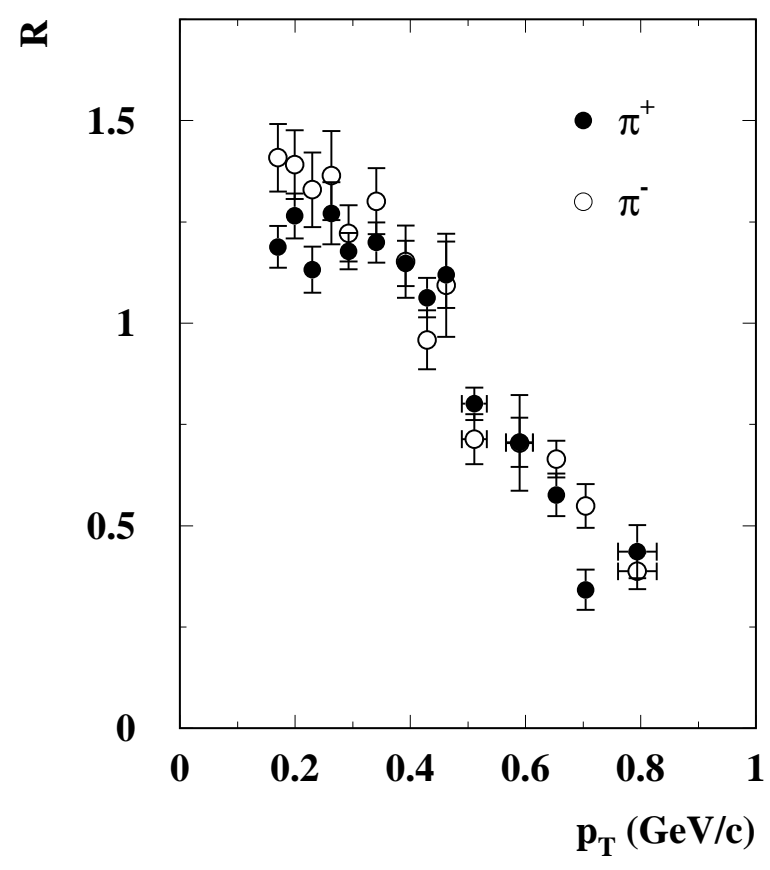

Figure 3.8: Yield ratio of pions emitted to opposite directions in the reaction plane $\left(\mathrm{R}=\mathrm{N}_{p} / \mathrm{N}_{t}\right)$ as a function of transverse momentum. $\mathrm{N}_{p}$ and $\mathrm{N}_{t}$ correspond to the number of pions emitted within $\phi= \pm 45^{0}$ (with respect to the reaction plane) towards the projectile and target side, respectively. The pions are measured at $\Theta_{l a b}=84^{0}$ (corresponding to normalized rapidities of $\left.0.01<y / y_{\text {proj }}<0.1\right)$ in peripheral $\mathrm{Au}+\mathrm{Au}$ collisions at $1 \mathrm{AGeV}$ 71].
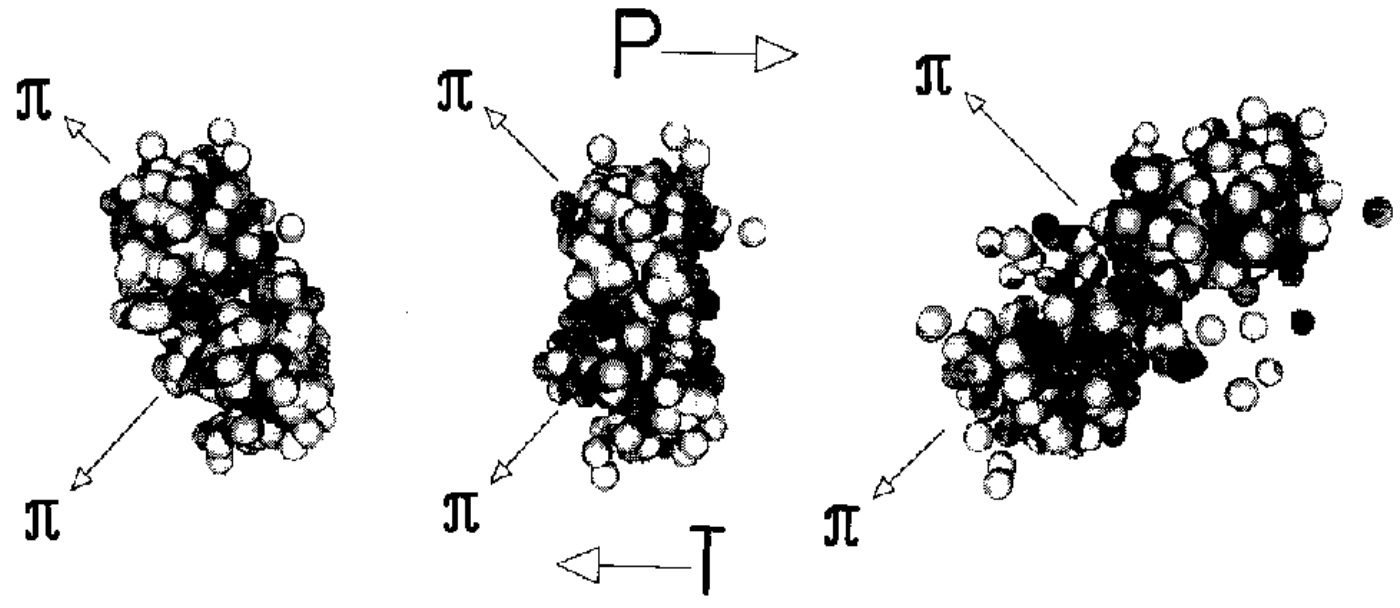

Figure 3.9: Sketch of an $\mathrm{Au}+\mathrm{Au}$ collision at $1 \mathrm{AGeV}$ with an impact parameter of $\mathrm{b}=7 \mathrm{fm}$ as calculated by the QMD transport code 99. The snapshots are taken at $4 \mathrm{fm} / \mathrm{c}$ (left), $8 \mathrm{fm} / \mathrm{c}$ (middle) and $20 \mathrm{fm} . \mathrm{c}$ (right). The pions are emitted in the reaction plane at backward angles corresponding to the detector position. 
First observations of a preferred out-of-plane emission of charged and neutral pions ("squeeze-out") were reported by the KaoS and TAPS collaborations 100, 101. The azimuthal anisotropy was most pronounced for pions with high transverse momenta in semicentral collisions. The experiments investigated $\mathrm{Au}+\mathrm{Au}$ collisions at $1 \mathrm{AGeV}$.

Recently, the pion azimuthal emission pattern has been studied in detail for $\mathrm{Bi}+\mathrm{Pb}$ collisions as a function of beam energy and impact parameter by the KaoS-Collaboration [102. The experiment was focused on the study of the pion emission perpendicular to the reaction plane and therefore the acceptance of the spectrometer covered midrapidity only.

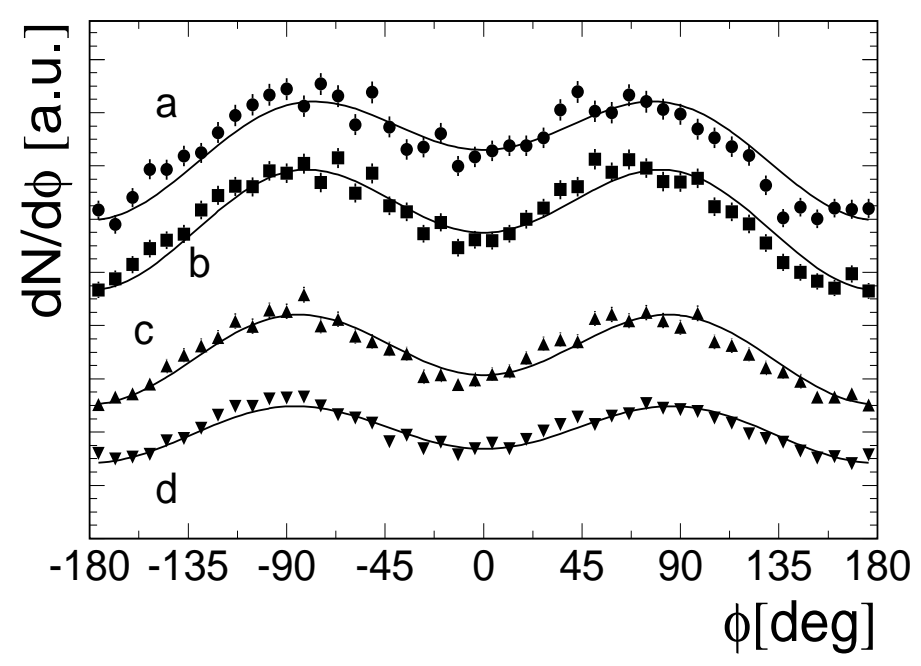

Figure 3.10: Azimuthal distributions of $\pi^{+}$ measured for semi-central $\mathrm{Bi}+\mathrm{Pb}$ collisions at $\mathrm{E}=700 \mathrm{AMeV}$ 102. The different symbols correspond to different bins in transverse momentum: (a) $240-300 \mathrm{MeV} / \mathrm{c}$, (b) $300-360 \mathrm{MeV} / \mathrm{c}$, (c) $360-420 \mathrm{MeV} / \mathrm{c}$ and (d) $420-480 \mathrm{MeV} / \mathrm{c}$. The linear ordinate starts at zero, the data are not corrected for the accuracy of the reaction plane determination. The solid lines are fits to the data (see text).

Fig. 3.10 shows the azimuthal distributions of $\pi^{+}$measured for semi-central $\mathrm{Bi}+\mathrm{Pb}$ collisions at $\mathrm{E}=$ $700 \mathrm{AMeV}$. The different symbols correspond to different bins in transverse momentum. The distributions show maxima at azimuthal angles of $\phi= \pm 90^{\circ}$ which is perpendicular to the reaction plane. The solid lines in Fig. 3.10 correspond to the parameterization

$$
N(\phi) \propto 1+a_{1} \cos \phi+a_{2} \cos 2 \phi
$$

The parameter $a_{1}$ quantifies the in-plane emission of the particles parallel $\left(a_{1}>1\right)$ or antiparallel $\left(a_{1}<1\right)$ to the impact parameter vector, whereas $a_{2}$ stands for an elliptic emission pattern which may be aligned with the event plane $\left(a_{2}>0\right)$ or oriented perpendicular to the event plane $\left(a_{2}<0\right)$. The parameters were determined by a fit to the data and corrected for the uncertainty in the reaction plane reconstruction by $a_{1,2}^{\prime}=a_{1,2} /\langle\cos 2 \Delta \phi\rangle$. The values of $\langle\cos 2 \Delta \phi\rangle$ have been determined by a Monte Carlo simulation and vary between 0.3 for peripheral and central collision and 0.5 for semi-central collisions (for details see 102).

The strength of the azimuthal anisotropy is given by the ratio $R_{N}^{c o r r}$ which is the number of particles emitted perpendicular to the event plane divided by the number of particles emitted parallel to the event plane (for $a_{2}<0$ ):

$$
R_{N}^{c o r r}=\frac{N\left(+90^{0}\right)+N\left(-90^{0}\right)}{N\left(0^{0}\right)+N\left(180^{0}\right)}=\frac{1-a_{2}^{\prime}}{1+a_{2}^{\prime}}
$$

The asymmetry parameter increases with pion transverse momentum. This is demonstrated in Fig. 3.11 which shows the $R_{N}^{\text {corr }}$ as a function of transverse momentum for $\mathrm{Au}+\mathrm{Au}$ and $\mathrm{Bi}+\mathrm{Bi}$ collisions at $1 \mathrm{AGeV}$. The strength of the pion azimuthal anisotropy is almost constant for impact parameters between $2.5 \mathrm{fm}$ and $10 \mathrm{fm}$ [102]. In contrast, the proton azimuthal anisotropy increases with increasing impact parameter whereas the dependence of $R_{N}^{c o r r}$ on transverse momentum and beam energy is similar for protons and pions [92]. According to transport calculations, the preferential emission perpendicular to the reaction plane is caused by rescattering and absorption in the spectator fragments 103. Although the models predict or reproduce the out-of-plane emission of pions qualitatively, a quantitative agreement has not been reached 


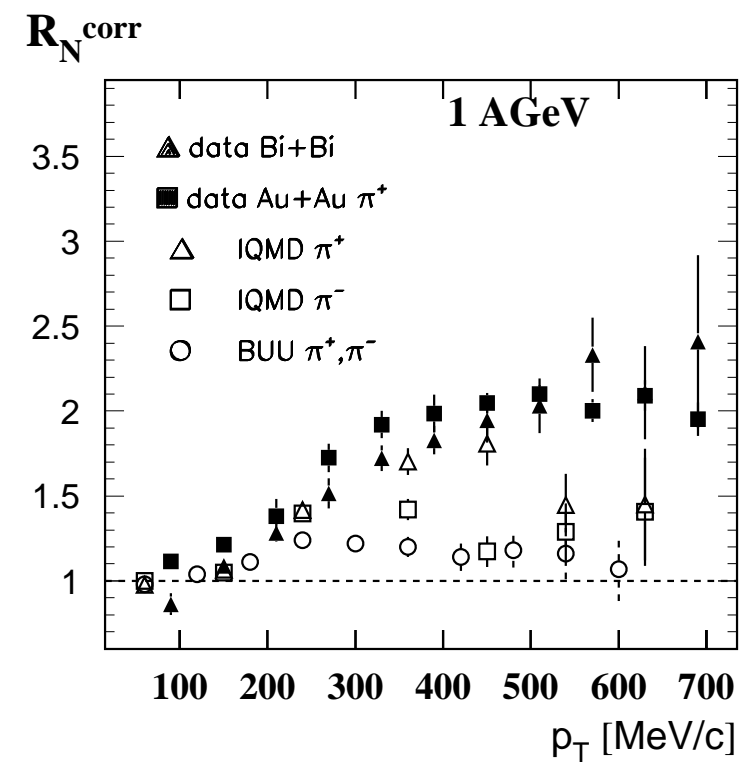

Figure 3.11: The anisotropy ratio for midrapidity pions from semi-central ( $\mathrm{b} \approx 7.5 \mathrm{fm}) \mathrm{Au}+\mathrm{Au}$ and $\mathrm{Bi}+\mathrm{Bi}$ collisions at $1 \mathrm{AGeV}$ (full symbols) as a function of transverse momentum 102. The model calculations (open symbols) are performed at $5 \leq \mathrm{b} \leq 10$ (IQMD 63]) and $7 \leq \mathrm{b} \leq 9$ (BUU [97]).

up to now. This means that the emission of pions from a hot nuclear medium is not yet well understood within the framework of transport model calculations.

The enhancement at $90^{\circ}$ in the polar angle distribution of the pions found in central collisions (see Fig. 3.6) may be another manifestation of the same phenomenon. It is a challenge for transport models to describe both the polar and azimuthal distribution of pions quantitatively and then answer the following questions: Is the pion azimuthal asymmetry due to an enhancement perpendicular the event plane or due to a suppression in the event plane? Are the pions absorbed or rescattered? Is absorption the reason for the reduction of the mean pion multiplicity per nucleon in $\mathrm{A}+\mathrm{A}$ with respect to $\mathrm{N}+\mathrm{N}$ collisions?

The experimental and theoretical studies of the pion azimuthal emission pattern at LBL/SIS energies can be summarized as follows: The anisotropies are probably caused mainly by the pion final-state interaction (rescattering and reabsorption). Low energy pions (which dominate the total pion yield) show a weak "squeeze-out" signal and weak antiflow for peripheral collisions. The pronounced out-of-plane emission of high-energy pions indicates that they freeze-out while the spectators are still close to the partizipant zone. The in-plane flow of high-energy pions is an additional signature for an early emission time. This finding is supported by an analysis of the $\pi^{-} / \pi^{+}$ratio as discussed in the next section.

Indications of azimuthal anisotropies in the pion emission pattern have also been found in $\mathrm{Au}+\mathrm{Au}$ collisions at AGS 104]. Data on the elliptic and directed flow of pions and protons measured in $\mathrm{Pb}+\mathrm{Pb}$ collisions at $158 \mathrm{AGeV}$ have been very recently published by Na49 105].

\subsection{Pion isospin ratios}

The multiplicity ratio of negatively to positively charged pions for $\mathrm{Au}+\mathrm{Au}$ collisions at $1 \mathrm{AGeV}$ was found to be $n_{\pi^{-}} / n_{\pi^{+}}=1.94 \pm 0.05$ [71] and $n_{\pi^{-}} / n_{\pi^{+}}=1.95 \pm 0.04 \pm 0.3$ [20]; the latter value is obtained when assuming a linear dependence on $\mathrm{A}_{\text {part }}$. According to the isospin decomposition taking into account $\Delta_{33}(1232)$ and $\mathrm{N}^{*}$ (1440) resonances the expected ratio is $n_{\pi^{-}} / n_{\pi^{+}}=1.90$ [61]. However, the $\pi^{-} / \pi^{+}$ratio is found to be not constant as a function of the pion transverse momentum. The pion low- $\mathrm{p}_{T}$ enhancement is much more pronounced for $\pi^{-}$than for $\pi^{+}$mesons. This effect can be seen in Fig. 3.12, Fig. 3.13 and Fig. 3.14 which 


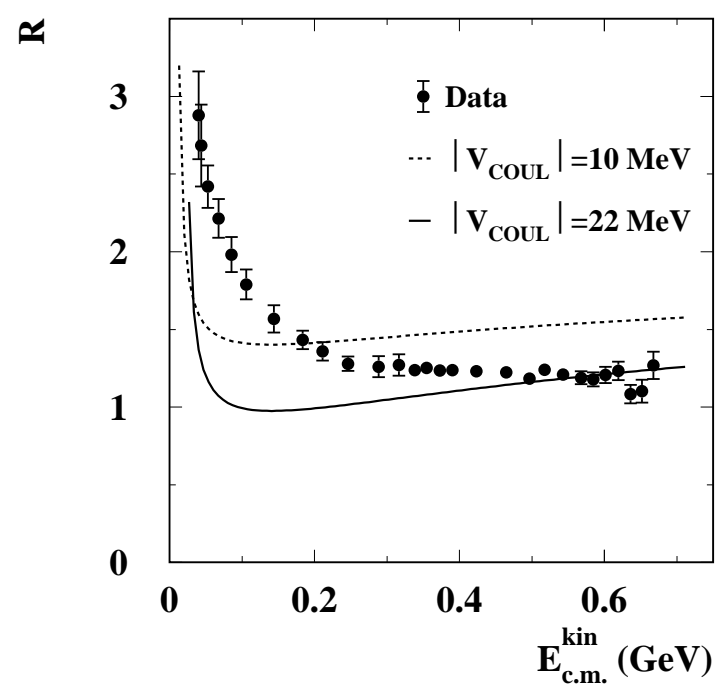

Figure 3.12: $\pi^{-} / \pi^{+}$ratio for central $\mathrm{Au}+\mathrm{Au}$ collisions at $1 \mathrm{AGeV}$ as a function of the pion kinetic energy. The solid (dotted) curve shows the calculated ratio for a fixed Coulomb potential of $22 \mathrm{MeV}(10 \mathrm{MeV})$ 71.

show the $\pi^{-} / \pi^{+}$ratios as a function of $\mathrm{p}_{T}\left(\right.$ or $\left.\mathrm{m}_{T}\right)$ measured in central collisions of very heavy nuclei at 1.0, 10.7 and $158 \mathrm{AGeV}$, respectively [71, 24, 44, 106]. According to simulations with RQMD or RBUU transport codes, the increase of the $\pi^{-} / \pi^{+}$ratio towards smaller values of transverse mass is due to the Coulomb interaction between pions and the net charge of the participants [63, 107. It can be seen that the effect is largest for the lowest bombarding energy $(1 \mathrm{AGeV})$. At a beam energy of $158 \mathrm{AGeV}$ the $\pi^{-} / \pi^{+}$ ratio exceeds unity only for values of $\mathrm{m}_{T}-\mathrm{m}_{0}$ below $0.1 \mathrm{GeV}$ [106].

The pronounced enhancement of the $\pi^{-} / \pi^{+}$ratio at low pion energies as measured for $1 \mathrm{AGeV}$ has been exploited to determine the size of the pion emitting source 71 . The procedure is based on the picture, that the primordial $\pi^{-}$and the $\pi^{+}$spectra are shifted against each other by the Coulomb field of the fireball which changes its size slowly compared to the velocity of the pions. Hence, the effective Coulomb potential of the fireball is determined from the shift in energy of the charged pions. It turns out that the deduced Coulomb potential decreases with decreasing pion energy. The effective freeze-out radius $\mathrm{r}_{e f f}$ is derived from the Coulomb potential $\mathrm{V}\left(\mathrm{r}_{e f f}\right)=\mathrm{e}^{2} \mathrm{Z} / \mathrm{r}_{\text {eff }}$ with $\mathrm{Z}$ the measured number of participating protons (which are the charged constituents of the fireball). Within the framework of this analysis a value of $\mathrm{r}_{\text {eff }}=$ $7.2 \pm 1.1 \mathrm{fm}$ has been determined for the freeze-out radius of high-energy pions in central $\mathrm{Au}+\mathrm{Au}$ collsions at $1 \mathrm{AGeV}[71]$. 

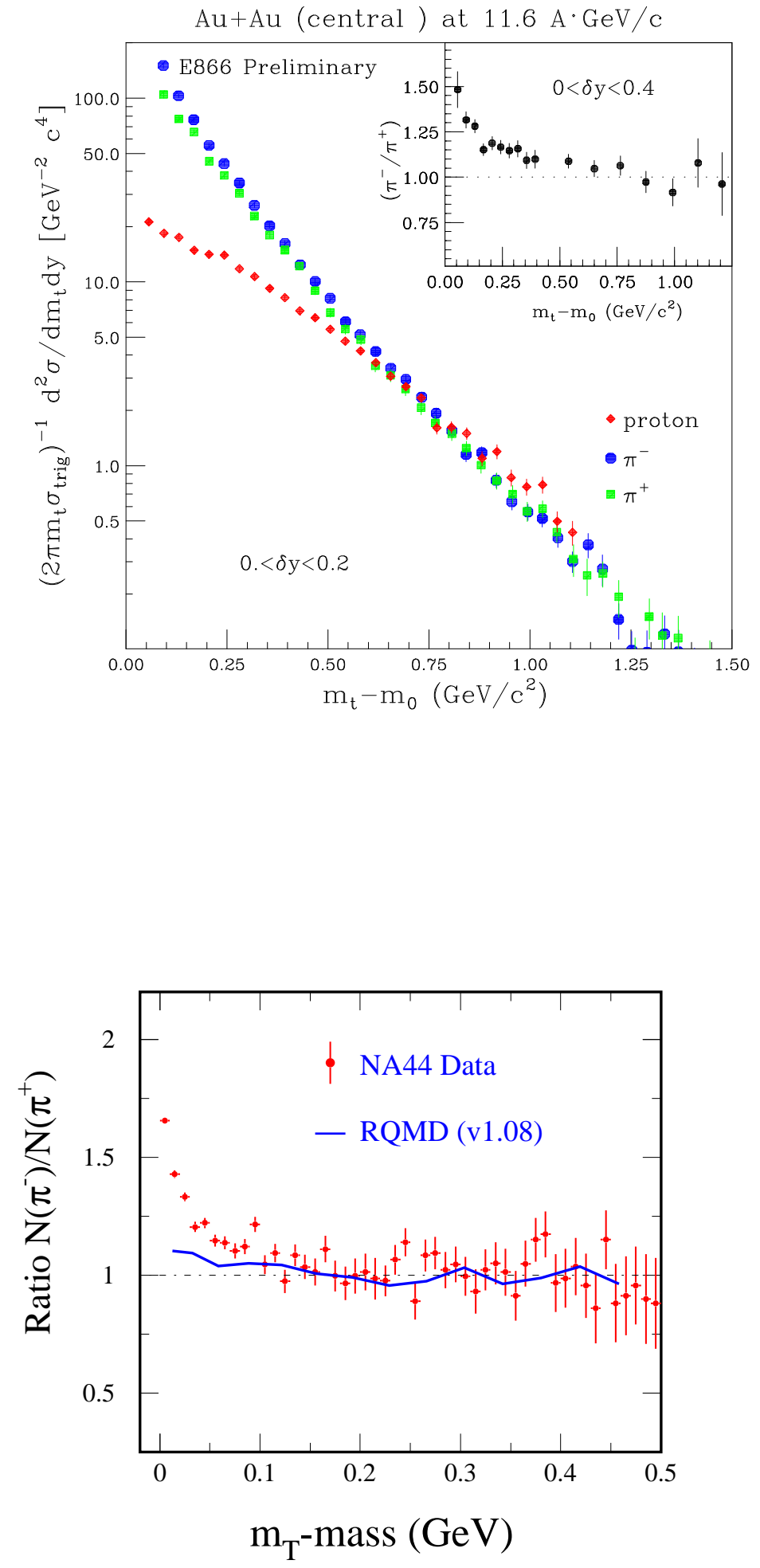

Figure 3.13: Transverse mass spectra of protons and pions measured in central $\mathrm{Au}+\mathrm{Au}$ collisions at 10.7 AGeV around midrapidity 24. The insert shows the corresponding $\pi^{-} / \pi^{+}$ ratio.
Figure 3.14: $\pi^{-} / \pi^{+}$ratio as a function of transverse mass measured in central $\mathrm{Pb}+\mathrm{Pb}$ collisions at 158 $\mathrm{AGeV}$ 106. 


\section{Chapter 4}

\section{Strangeness Production}

The production of strange particles in nucleus-nucleus collisions is of particular interest as the heavy strange (and antistrange) quarks do not exist prior to the collision. The annihilation of a produced $s \bar{s}$ pair is unlikely as long as their abundance is low. Therefore, the strange (and antistrange) quarks "live" in hadrons until they disappear by weak decays. The $\mathrm{K}^{+}$meson, for example, leaves the hot and dense reaction zone nearly undisturbed because of its long mean free path in nuclear matter $(\lambda \approx 5 \mathrm{fm})$. The corresponding $\mathrm{K}^{+} \mathrm{N}$ cross section is almost entirely due to the elastic scattering process $\left(\sigma_{K^{+} N \rightarrow K^{+} N} \approx 12\right.$

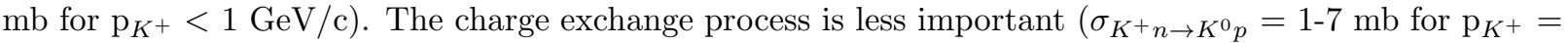
$0.2-0.6 \mathrm{GeV} / \mathrm{c}$ ) 108 .

In heavy ion collisions at BEVALAC/SIS energies, kaons are produced near or below the kinematical thresholds which correspond to kinetic beam energies of $1.58 \mathrm{GeV}$ (for $\mathrm{NN} \rightarrow \mathrm{K}^{+} \Lambda \mathrm{N}$ ) and $2.5 \mathrm{GeV}$ (for $\left.\mathrm{NN} \rightarrow \mathrm{K}^{+} \mathrm{K}^{-} \mathrm{N}\right)$. For central nucleus-nucleus collisions at "subthreshold" beam energies, experimental results suggest that a major fraction of the kaons is created in processes which require multiple interactions of the participant nucleons [51, 109, 110, 111]. Within the framework of microscopic transport models, these kaons are produced in secondary collisions like $\Delta \mathrm{N} \rightarrow \mathrm{KYN}$ and $\pi \mathrm{N} \rightarrow \mathrm{KYN}$ with $\mathrm{Y}=\Lambda, \Sigma[112,113,114$, 115, 116, 118. Sequential processes occur preferentially at high baryonic densities and therefore the $\mathrm{K}^{+}$yield is expected to be sensitive to nuclear matter properties [117. This sensitivity is enhanced at bombarding energies far below the $\mathrm{K}^{+}$threshold. For $\mathrm{Au}+\mathrm{Au}$ at $1 \mathrm{AGeV}$, transport codes predict a measurable effect on the $\mathrm{K}^{+}$production cross section when considering different nuclear equation of states [117, 116, 119]. However, the reliability of the model calculations still suffers from an insufficient understanding (and the simplified treatment) of the in-medium nucleon-nucleon interactions and from the lack of information on $\Delta \mathrm{N} \rightarrow \mathrm{KYN}$ reactions. Nevertheless, nuclear matter properties can be explored by detailed comparisons of specific model predictions to experimental data as function of energy and system size.

In the last years, the question of in-medium modifications of hadron properties has attracted increasing interest. Calculations based on chiral Lagrangians predict an in-medium kaon-nucleon potential which is weakly repulsive for kaons but strongly attractive for antikaons [120, 121, 122]. Recent transport model calculations, which incorporate these medium effects, find a considerable impact on the $\mathrm{K}^{+}$and $\mathrm{K}^{-}$yields and on their azimuthal angular distributions for nucleus-nucleus collisions at SIS energies $123,124,125$, 126, 127. In particular, the yield of antikaons is expected to be significantly enhanced in dense nuclear matter. Indeed, experimental results on $\mathrm{K}^{-}$production and on $\mathrm{K}^{+}$directed flow in $\mathrm{Ni}+\mathrm{Ni}$ collisions were interpreted as indication for in-medium modifications of kaon properties [55, 111, 128].

At ultrarelativistic energies, strangeness production is considered as a signal from the quark-gluon plasma (QGP) phase. An extraordinary increase of the strange particle yield is expected if the fireball has spent a non negligible amount of time in the deconfined phase. During this period, chemical equilibrium abundances of $\mathrm{s}, \mathrm{u}, \mathrm{d}$ quarks are reached faster than in an hadronic environment: in the QGP phase the temperature is comparable to the strange quark mass whereas in the hadronic phase the production of strange hadrons is suppressed by their large masses. This is, however, not the only scenario for an enhanced production of strange (with respect to nonstrange) particles. We have mentioned for example the 
effective lowering of the production threshold by secondary interactions. Experimentally the strangeness enhancement has been established in nucleus-nucleus collisions at SIS, AGS and SPS energies. It is a challenge to unravel the different underlying dynamics at the different energies.

In the following sections we review the existing experimental data on the production cross sections of kaons and on their spectral distributions. The low energy data and their impact on the determination of nuclear matter properties will be considered first (section 4.1 through 4.5). In section 4.6 the production of K-mesons at AGS and SPS energies will be reviewed. Multistrange hadrons will be covered together with production of antibaryons in a separate chapter.

\subsection{Kaon production probabilities}

First experiments on strangeness production in nucleus-nucleus collisions were performed in the early 1980's at the LBL in Berkeley 129, 130, 54] and at the JINR in Dubna [131. Inclusive $\mathrm{K}^{+}$production cross sections $d^{2} \sigma / d p d \Omega$ were measured in $\mathrm{Ne}+\mathrm{NaF}$ and $\mathrm{Ne}+\mathrm{Pb}$ collisions at $2.1 \mathrm{AGeV}$ between $15^{\circ}<\Theta_{\text {lab }}<80^{\circ} 129$.

The second generation experiments at SIS/GSI have extended the study of $\mathrm{K}^{+}$production to very heavy collision systems and to beam energies far below the threshold for free nucleon-nucleon collisions which is $1.58 \mathrm{GeV}$ [21, 51, 52, 111, 109, 110, 128]. Table 4.1 presents a compilation of total $\mathrm{K}^{+}$cross sections measured in nucleus-nucleus collisions at energies between 0.8 and $2 \mathrm{GeV}$ per nucleon. The data were taken mostly within a restricted range of emission angles and momenta. The cited total $\mathrm{K}^{+}$cross sections were calculated by extrapolating to full phase space assuming a Maxwell-Boltzmann distribution and isotropic emission (the validity of the second assumption will be discussed in section 4.3).

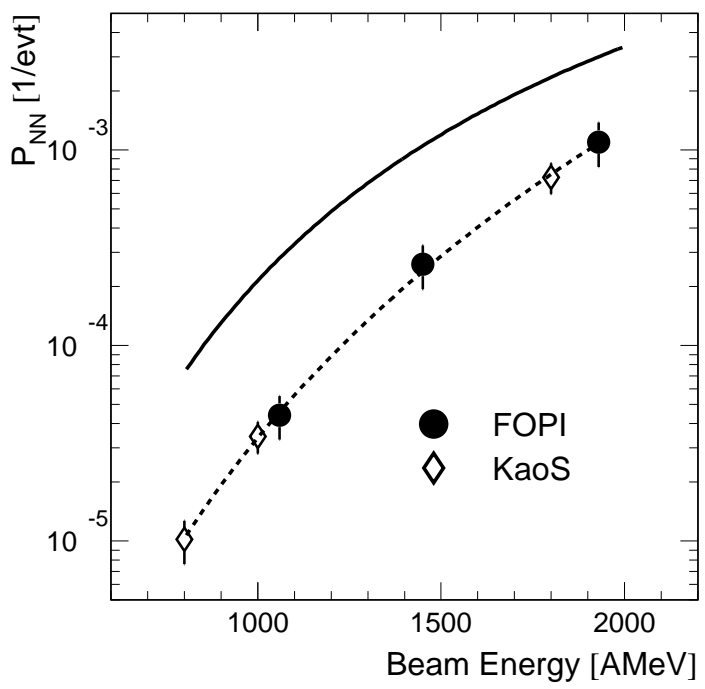

Figure 4.1: $\mathrm{K}^{+}$production probability $\left(\mathrm{P}_{N N}=\right.$ multiplicity per participating nucleon) measured in $\mathrm{Ni}+\mathrm{Ni}$ collisions as a function of beam energy (full symbols: [21], open symbols: [111]). Dashed line: $\mathrm{P}_{N N} \propto \mathrm{E}_{\text {beam }}^{5.3 \pm 0.3}$. The solid line represents the production probability expected for pions and eta-mesons (when corrected for the threshold energies) according to an empirical scaling by Metag ([132], see Fig. 5.3). Taken from [21].

For $\mathrm{Ni}+\mathrm{Ni}$ collisions, the excitation function of $\mathrm{K}^{+}$production at beam energies below and near threshold can be parameterized by $\mathrm{P}_{K^{+}} \propto \mathrm{E}_{\text {beam }}^{\alpha_{E}}$ with $\alpha_{E}=5.3 \pm 0.3$ (see Fig. 4.1). $\mathrm{P}_{K^{+}}$is the $\mathrm{K}^{+}$production probability per participating nucleon. The data are taken from [21, 111]. The corresponding exponent $\alpha_{E}$ in $\mathrm{p}+\mathrm{p} \rightarrow K^{+}+\mathrm{X}$ reactions near threshold is much larger $(\approx 18)$. In fact, a value of $\alpha_{E}=5.3$ is found for the $\mathrm{p}+\mathrm{p}$ reaction only at beam energies between 2.0 and $2.5 \mathrm{GeV}$ i.e. more than $400 \mathrm{MeV}$ above threshold.

The average $\mathrm{K}^{+}$multiplicity per participating nucleon was studied as a function of the mass of the colliding system for a beam energy of $1 \mathrm{AGeV}$. It was calculated from the $\mathrm{K}^{+}$production cross section according to $\mathrm{n}_{K^{+}}(\mathrm{A})=\sigma^{K^{+}} / \sigma_{R}$ with the reaction cross section $\sigma_{R}=4 \pi\left(\mathrm{r}_{o} \mathrm{~A}^{1 / 3}\right)^{2}\left(\mathrm{r}_{o}=1.2 \mathrm{fm}\right)$. The $\mathrm{K}^{+}$ production cross section was measured for $\mathrm{C}+\mathrm{C}, \mathrm{Ne}+\mathrm{NaF}, \mathrm{Ni}+\mathrm{Ni}$ and $\mathrm{Au}+\mathrm{Au}$ [52, 133, 110, 111]. Fig. 4.2 shows the $\mathrm{K}^{+}$multiplicity per participating nucleon $\mathrm{n}_{K^{+}} /<\mathrm{A}_{\text {part }}>$ with $<\mathrm{A}_{\text {part }}>=\mathrm{A} / 2$ according to the geometrical model for symmetric collisions. The increase of $\mathrm{n}_{K^{+}} /<\mathrm{A}_{\text {part }}>$ with the mass of the collision system is a clear experimental signature for kaon production via collective processes. According 
Table 4.1: Inclusive $\mathrm{K}^{+}$production cross sections and inverse slope parameters $\mathrm{T}$ from symmetric nucleusnucleus collisions. The values are determined by fitting the data with a Maxwell Boltzmann distribution $\mathrm{d}^{3} \sigma / \mathrm{dp}^{3} \propto \exp (-\mathrm{E} / \mathrm{T})$ and integrating the fitted curve over momentum. The total cross section is calculated assuming isotropic emission in $4 \pi$. The $\mathrm{Au}+\mathrm{Au}$ cross sections agree within the error bars, the slopes do not. However, the dataset of Ref. [51 consists of about $100 \mathrm{~K}^{+}$whereas the results of Ref. [110] are based on about $10^{4} \mathrm{~K}^{+}$mesons. The data marked with $(*)$ are preliminary.

\begin{tabular}{|c|c|c|c|c|c|}
\hline $\mathrm{A}+\mathrm{A}$ & $\mathrm{E}(\mathrm{AGeV})$ & $\Theta_{l a b}(\operatorname{deg})$ & $4 \pi \times \mathrm{d} \sigma / d \Omega(\mathrm{mb})$ & $\mathrm{T}(\mathrm{MeV})$ & Ref. \\
\hline $\mathrm{C}+\mathrm{C}$ & $1.0(*)$ & 44 & $0.1 \pm 0.03$ & $61 \pm 5$ & |133 \\
\hline $\mathrm{C}+\mathrm{C}$ & $1.8(*)$ & 40 & $3.3 \pm 1$ & $73 \pm 6$ & 133 \\
\hline$\overline{\mathrm{Ne}+\mathrm{NaF}}$ & $\overline{1.0}$ & $\overline{44}$ & $0.33 \pm 0.1$ & $61 \pm 6$ & 52 \\
\hline$\overline{\mathrm{Ne}+\mathrm{NaF}}$ & 2.0 & $\overline{44}$ & $20 \pm 10$ & $\overline{79 \pm 6}$ & 52 \\
\hline$\overline{\mathrm{Ne}+\mathrm{NaF}}$ & 2.1 & $15-80$ & $23 \pm 8$ & $\overline{122}$ & [129] \\
\hline $\mathrm{Ni}+\mathrm{Ni}$ & 0.8 & 44 & $0.85 \pm 0.2$ & $59 \pm 6$ & |111 \\
\hline $\mathrm{Ni}+\mathrm{Ni}$ & 1.0 & 44 & $2.7 \pm 0.5$ & $\overline{75 \pm 6}$ & |111 \\
\hline $\mathrm{Ni}+\mathrm{Ni}$ & 1.8 & 44 & $57 \pm 15$ & $88 \pm 7$ & |111] \\
\hline $\mathrm{Au}+\mathrm{Au}$ & 1.0 & 44 & $41 \pm 11$ & $67 \pm 5$ & 51 \\
\hline $\mathrm{Au}+\mathrm{Au}$ & $1.0(*)$ & $34,44,54,84$ & $26 \pm 5$ & $85 \pm 6$ & $\mid 110$ \\
\hline $\mathrm{Bi}+\mathrm{Pb}$ & 0.8 & 44 & $9.4 \pm 4.9$ & $64 \pm 11$ & 109 \\
\hline
\end{tabular}

to model calculations the kaons are produced predominantly in $\Delta$-nucleon and pion-nucleon collisions $1112,118,123,135$.

Kaon production was also studied as a function of impact parameter. It was found that the $\mathrm{K}^{+}$yield normalized to the number of participating nucleons is enhanced in central as compared to peripheral nucleus-nucleus collisions [51]. Fig. 4.3 shows the meson multiplicities $n_{K^{+}}$and $n_{\pi^{+}}$per participating nucleon $\left(\mathrm{A}_{\text {part }}\right)$ as a function of $\mathrm{A}_{\text {part }}$ measured by the KaoS Collaboration for $\mathrm{Au}+\mathrm{Au}$ at $1 \mathrm{AGeV}$ [110]. The $\pi^{+}$muliplicity per $\mathrm{A}_{\text {part }}$ is roughly constant (circles) whereas $\mathrm{n}_{K^{+}}$increases according to $\mathrm{A}_{\text {part }}^{\alpha_{A}}$ with $\alpha_{A}=1.8 \pm 0.15$. Values of $\alpha_{A}$ larger than unity indicate that kaons are produced in multiple collisions of participants. The $\mathrm{A}_{\text {part }}$-dependence of $\mathrm{n}_{K^{+}}\left(\mathrm{A}_{\text {part }}\right)$ is more pronounced than the A-dependence of the average kaon multiplicity per participating nucleon for different $\mathrm{A}+\mathrm{A}$ collisions as shown in Fig. 4.2 .

The $\mathrm{K}^{+}$scaling factor $\alpha_{A}$ has been also measured for $\mathrm{Ni}+\mathrm{Ni}$ collisions as a function of the bombarding energy (see Fig. 4.4] 111]. Values of $\alpha_{A}=1.9 \pm 0.25,1.7 \pm 0.25$ and $1.65 \pm 0.15$ have been determined for $0.8,1.0$ and $1.8 \mathrm{AGeV}$, respectively. Thus $\alpha_{A}$ does not vary significantly with bombarding energy between 0.8 and $1.8 \mathrm{AGeV}$ for intermediate mass systems. Hovever, for $\mathrm{K}^{+}$production in $\mathrm{Bi}+\mathrm{Pb}$ collisions at 0.8 $\mathrm{AGeV}$ a value of $\alpha_{A}=2.26 \pm 0.14$ was found 109. This value is larger than the one obtained for $\mathrm{Au}+\mathrm{Au}$ at $1 \mathrm{AGeV}$ (see above) indicating a weak increase of $\alpha_{A}$ with decreasing beam energy for very heavy collision systems.

The data on $\mathrm{K}^{-}$production in nucleus-nucleus collisions are rather scarce. Pioneering experiments at the BEVALAC studied $\mathrm{K}^{-}$yields in the system $\mathrm{Si}+\mathrm{Si}$ at beam energies of $1.16-2.0 \mathrm{AGeV}$ and in $\mathrm{Ne}+\mathrm{NaF}$ at $2.0 \mathrm{AGeV}$ at laboratory angles of $0^{\circ}$ 54, 136. Similar experiments $\left(\right.$ at $\Theta_{l a b}=0^{0}$ ) were performed with the Fragment Separator (FRS) at SIS/GSI [55, 137, 138]. Fig. 4.5 shows the $\mathrm{K}^{-}$invariant production cross section for $\mathrm{Ne}+\mathrm{NaF}$ (at $1.34-1.94 \mathrm{AGeV}$ ) and $\mathrm{Ni}+\mathrm{Ni}$ collisions (at $1.22-1.85 \mathrm{AGeV}$ ) 138. The spectral distributions fall off exponentially as indicated by the dashed and solid lines. The values of the inverse slope parameters are listed in Table 4.2. They seem to be independent of beam energy and projectile mass (within the large error bars). The data allow to extract an excitation function $\sigma^{K^{-}} \propto \mathrm{E}_{\text {beam }}^{\alpha_{E}}$ with $\alpha_{E} \approx 10$.

The mass dependence of $\mathrm{K}^{-}$production can also be estimated from the FRS data (some data given in [55] have been revised in [138]). The ratio of the $\mathrm{K}^{-}$production cross sections measured in $\mathrm{Ni}+\mathrm{Ni}$ and $\mathrm{Ne}+\mathrm{NaF}$ was found to be $\sigma_{N i}^{K^{-}} / \sigma_{N e}^{K^{-}} \approx 10$ [138. When correcting for the geometrical reaction cross section $\sigma_{R}=4 \pi\left(\mathrm{r}_{o} \mathrm{~A}^{1 / 3}\right)^{2}$ one obtains the ratio of multiplicities $\mathrm{M}_{N i}^{K^{-}} / \mathrm{M}_{N e}^{K^{-}} \approx 5$ corresponding to a scaling 


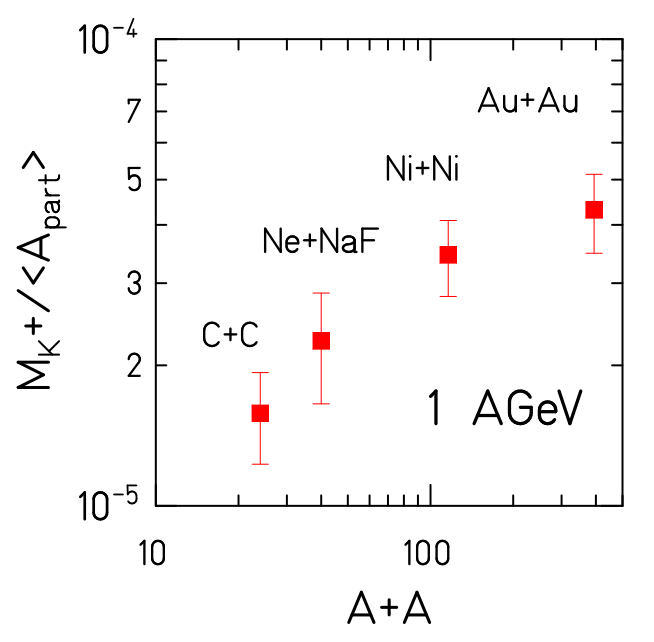

Figure 4.2: $\mathrm{K}^{+}$multiplicity per average number of participating nucleons as a function of the mass of nuclei colliding at $1 \mathrm{AGeV}[52,110,111,133]$.

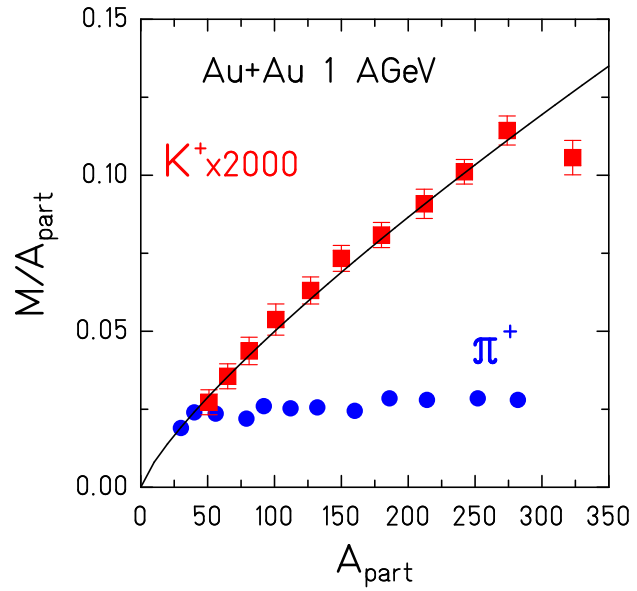

Figure 4.3: $\mathrm{K}^{+}$and $\pi^{+}$multiplicity per number participating nucleons $\mathrm{A}_{\text {part }}$ as a function of $\mathrm{A}_{\text {part }}$ for $\mathrm{Au}+\mathrm{Au}$ collisions at $1 \mathrm{AGeV}$ [73, 110] (preliminary).

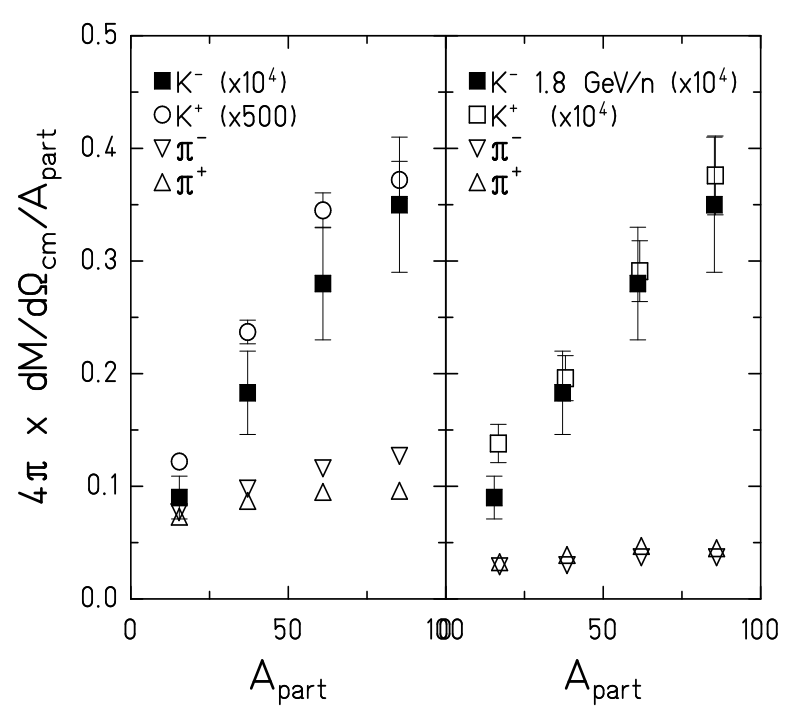

Figure 4.4: Kaon and pion multiplicities per number participating nucleons $\mathrm{A}_{\text {part }}$ as a function of $\mathrm{A}_{\text {part }}$ for $\mathrm{Ni}+\mathrm{Ni}$ collisions. Left panel: $\mathrm{K}^{-}, \mathrm{K}^{+}, \pi^{+}, \pi^{-}$for a beam energy of $1.8 \mathrm{AGeV}$. Right panel: $\mathrm{K}^{-}$at $1.8 \mathrm{AGeV}$ and $\mathrm{K}^{+}$, $\pi^{+}, \pi^{-}$at $1.0 \mathrm{AGeV}$. 1111 

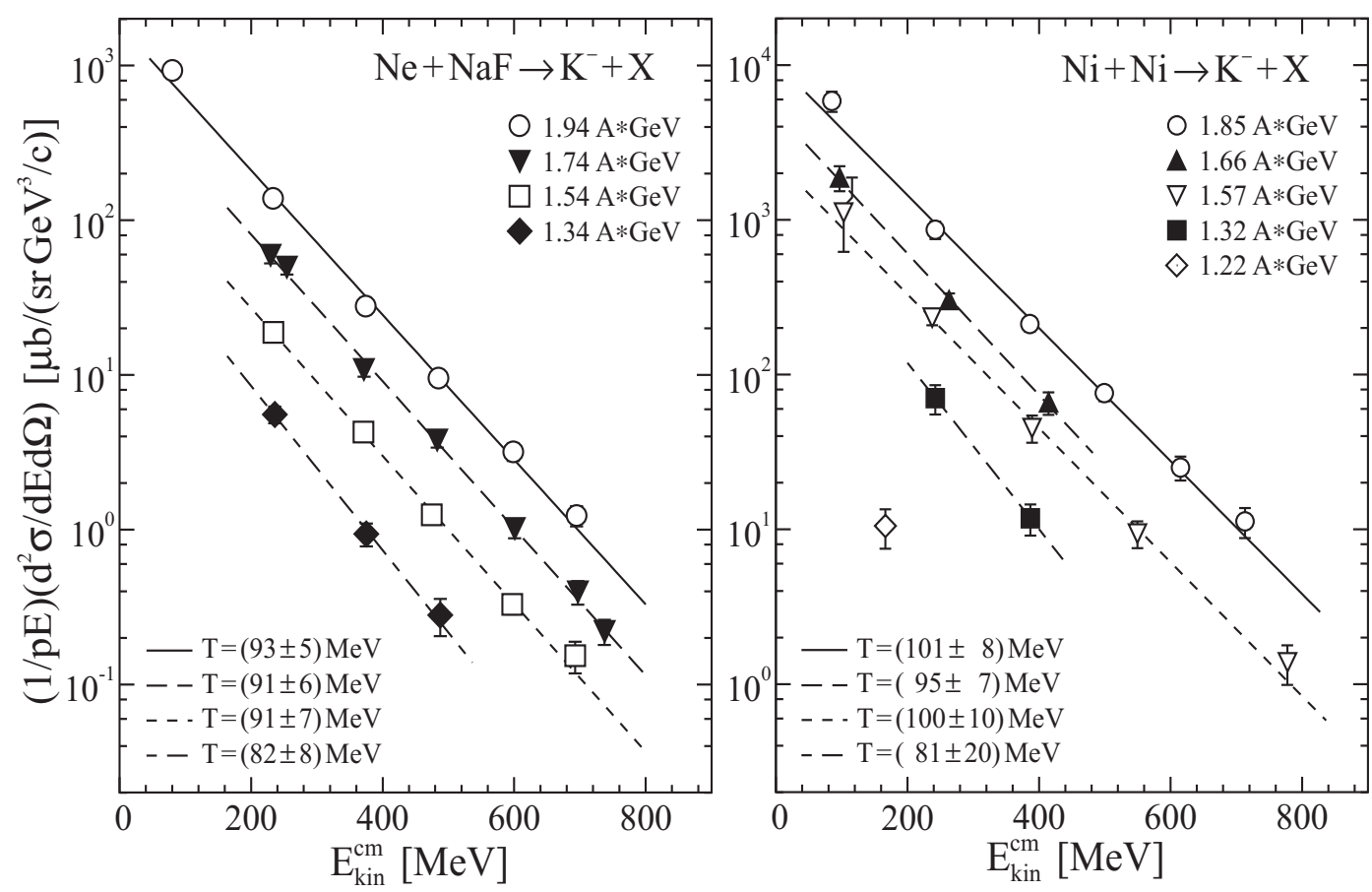

Figure 4.5: Invariant $\mathrm{K}^{-}$production cross section as a function of the $\mathrm{K}^{-}$c.m. kinetic energy for the reactions $\mathrm{Ne}+\mathrm{NaF}$ and $\mathrm{Ni}+\mathrm{Ni}$ at the bombarding energies indicated 138. The lines are exponential fits to the data points with inverse slope parameters $\mathrm{T}$ as indicated.

of $\mathrm{M}^{K^{-}} \propto \mathrm{A} \approx 1.5$.

Recently, $\mathrm{K}^{-}$production cross sections have been measured also around midrapidity $\left(\Theta_{c . m .} \approx 90^{\circ}\right)$ in $\mathrm{Ni}+\mathrm{Ni}$ and $\mathrm{C}+\mathrm{C}$ collisions at $1.8 \mathrm{AGeV}$ by the KaoS Collaboration 111, 134. From these data one can estimate a scaling according to $\mathrm{M}^{K^{-}} \propto \mathrm{A}^{1.6 \pm 0.2}$. This scaling behaviour is in agreement with the dependence of $\mathrm{K}^{-}$production on the number of participants as found in $\mathrm{Ni}+\mathrm{Ni}$ collisions for different impact parameters: $\mathrm{M}^{K^{-}} \propto \mathrm{A}_{\text {part }}^{\alpha_{A}}$ with $\alpha_{A}=1.8 \pm 0.3$ [11].

In $\mathrm{Ni}+\mathrm{Ni}$ collisions at $1.8 \mathrm{AGeV}$, the $\mathrm{K}^{-} / \mathrm{K}^{+}$ratio was found to be $4.8 \pm 2 \times 10^{-2} \sqrt[111]{ }$. In $\mathrm{C}+\mathrm{C}$ collisions at $1.8 \mathrm{AGeV}$, the preliminary value for the $\mathrm{K}^{-} / \mathrm{K}^{+}$ratio is approximately $2.5 \times 10^{-2} \not 134$. When increasing the mass of the collision system from $\mathrm{C}+\mathrm{C}$ to $\mathrm{Ni}+\mathrm{Ni}$, the $\mathrm{K}^{-}$yield increases by about a factor of 2 faster than the $\mathrm{K}^{+}$yield. Part of this $\mathrm{K}^{-}$enhancement may be due to the fact that at a beam energy of 1.8 $\mathrm{AGeV}$ the production of $\mathrm{K}^{-}$mesons is a subthreshold process which is more sensitive to the size of the collision system than $\mathrm{K}^{+}$production which is above threshold. Nevertheless, the experimental result is quite intriguing since one expects that due to $\mathrm{K}^{-}$absorption the $\mathrm{K}^{-}$yield increases slower than the $\mathrm{K}^{+}$ yield with increasing mass and size of the collision system [139].

A measurement of $\mathrm{K}^{+}$and $\mathrm{K}^{-}$at slightly higher $(3.65 \mathrm{AGeV})$ energies has been made available in reference [140]. The authors report ratios $\mathrm{K}^{+} / \pi^{+}=0.07$ and $\mathrm{K}^{-} / \mathrm{K}^{+}=0.07$ for $\mathrm{C}+\mathrm{C}$ collisions at midrapidity. A very similar value for the $\mathrm{K}^{-} / \mathrm{K}^{+}$ratio has been measured in proton-proton collisions at the same bombarding energy (see Fig. 4.17).

\subsection{Kaon spectral distributions}

At sufficiently low center-of-mass energies, particle emission is more or less isotropic. Under this condition the appropriate kinematical observable is the kinetic energy. Its distribution is a straight exponential in the case of a completely chaotic motion with different particles having the same slope factor which is simply the inverse temperature. Collective radial flow will modify the shape of such spectra. This effect increases with increasing mass of the particles with the consequence that particles with different masses will have 
Table 4.2: Inclusive $\mathrm{K}^{-}$production cross sections and inverse slope parameters $\mathrm{T}$ from symmetric nucleusnucleus collisions. The values are determined by fitting the data with a Maxwell Boltzmann distribution $\mathrm{d}^{3} \sigma / \mathrm{dp}^{3} \propto \exp (-\mathrm{E} / \mathrm{T})$ and integrating the fitted curve over momentum. The total cross section is calculated assuming isotropic emission in $4 \pi$. The data marked with $\left(^{*}\right)$ are preliminary.

\begin{tabular}{|c|c|c|c|c|c|}
\hline $\mathrm{A}+\mathrm{A}$ & $\mathrm{E}(\mathrm{AGeV})$ & $\Theta_{l a b}(\mathrm{deg})$ & $4 \pi \times \mathrm{d} \sigma / d \Omega(\mathrm{mb})$ & $\mathrm{T}(\mathrm{MeV})$ & Ref. \\
\hline $\mathrm{C}+\mathrm{C}$ & $1.8\left(^{*}\right)$ & 40 & $0.075 \pm 0.02$ & $60 \pm 5$ & {$[134]$} \\
\hline $\mathrm{Ne}+\mathrm{NaF}$ & 1.34 & 0 & - & $82 \pm 8$ & $138]$ \\
\hline $\mathrm{Ne}+\mathrm{NaF}$ & 1.54 & 0 & - & $91 \pm 7$ & $138]$ \\
\hline $\mathrm{Ne}+\mathrm{NaF}$ & 1.74 & 0 & - & $91 \pm 6$ & $138]$ \\
\hline $\mathrm{Ne}+\mathrm{NaF}$ & 1.94 & 0 & - & $93 \pm 5$ & {$[138]$} \\
\hline $\mathrm{Si}+\mathrm{Si}$ & 2.0 & 0 & & $103 \pm 7$ & {$[54]$} \\
\hline $\mathrm{Ni}+\mathrm{Ni}$ & 1.32 & 0 & - & $81 \pm 20$ & {$[138]$} \\
\hline $\mathrm{Ni}+\mathrm{Ni}$ & 1.57 & 0 & - & $100 \pm 10$ & {$[138]$} \\
\hline $\mathrm{Ni}+\mathrm{Ni}$ & 1.66 & 0 & - & $95 \pm 7$ & $138]$ \\
\hline $\mathrm{Ni}+\mathrm{Ni}$ & 1.85 & 0 & - & $101 \pm 8$ & {$[138]$} \\
\hline $\mathrm{Ni}+\mathrm{Ni}$ & 1.8 & 44 & $2.7 \pm 1.0$ & $90 \pm 15$ & $111]$ \\
\hline
\end{tabular}

different slope factors. Another complication in the interpretation of particle spectra is the effect induced by resonance decays which occur after freeze-out. At low (SIS) energies kaons are not affected by this phenomenon. Therefore, kaons are especially suited to study the interplay of chaotic and ordered motion.

The $\mathrm{K}^{+}$spectra measured at LBL/SIS energies were parameterized by Maxwell-Boltzmann distributions [52, 111]. The resulting inverse slope parameters as derived from inclusive differential cross sections at midrapidity are listed in Table 4.1 .

It was found that the $\mathrm{K}^{+}$inverse slope parameters agree with the those of high-energy pions (see tables 3.3 and 4.1). This accordance is demonstrated for different collision systems (at $1 \mathrm{AGeV}$ ) in Fig. 4.6 which shows the $\mathrm{K}^{+}$and $\pi^{+}$invariant cross section as a function of the total energy carried by the particle which includes the energy needed to produce the particle. The total energy is $\mathrm{E}_{t o t}=\mathrm{E}_{k i n}+\mathrm{m}_{\pi}$ for pions and $\mathrm{E}_{t o t}=\mathrm{E}_{k i n}+\mathrm{m}_{K}+\left(\mathrm{m}_{\Lambda}-\mathrm{m}_{N}\right)$ for the associated production of a $\mathrm{K}^{+}$. The differential cross sections of the different particle types coincide within a factor of about 2 in the interval in which their total energies overlap.

Spectral distributions of $\mathrm{K}^{+}$and pions were also measured as a function of collision centrality in $\mathrm{Au}+\mathrm{Au}$ collisions at a bombarding energy of $1 \mathrm{AGeV}$ (see Fig. 4.7). The inverse slope parameters both of highenergy pions and $\mathrm{K}^{+}$mesons increase with increasing centrality. The fact that particles with different masses exhibit very similar spectral slopes, indicates that the impact of collective flow and resonance decays is small. It rather suggests that the "temperature" (i.e. the energy in chaotic motion) of the system rises with the number of participant nucleons and thus with system size. Furthermore, these observations are a signature for a common freeze-out temperature realized probably in the early phase of the nuclear fireball with particle emission according to the phase space available.

According to RBUU calculations the $\mathrm{K}^{+}$mesons are produced in the high density phase $\left(\rho>2 \rho_{o}\right.$ in central $\mathrm{Au}+\mathrm{Au}$ collisions at $1 \mathrm{AGeV})$ and the $\mathrm{K}^{+}$yield reaches its final value after about $17 \mathrm{fm} / \mathrm{c} 123$. At this time the system starts to expand. The primordial kaon spectrum is relatively soft due to the limited phase space but will be modified by the $\mathrm{K}^{+}$propagation and rescattering in the nuclear medium. The kaons get (partially) thermalized by $\mathrm{K}^{+} \mathrm{N}$ rescattering which makes the spectrum harder 123 .

In $\mathrm{Ne}+\mathrm{NaF}$ collisions at $2 \mathrm{AGeV}$, invariant cross sections for kaon and pions production have been measured [52]. Inverse slope parameter of about $80 \mathrm{MeV}$ were found both for $\mathrm{K}^{+}$mesons and high-energy pions [52]. This result is at variance with the high $\mathrm{K}^{+}$"temperature" of $\mathrm{T}=122 \mathrm{MeV}$ reported for $\mathrm{Ne}+\mathrm{NaF}$ collisions at $2.1 \mathrm{AGeV}$ [129] which were interpreted as a signature for freeze-out during an early 

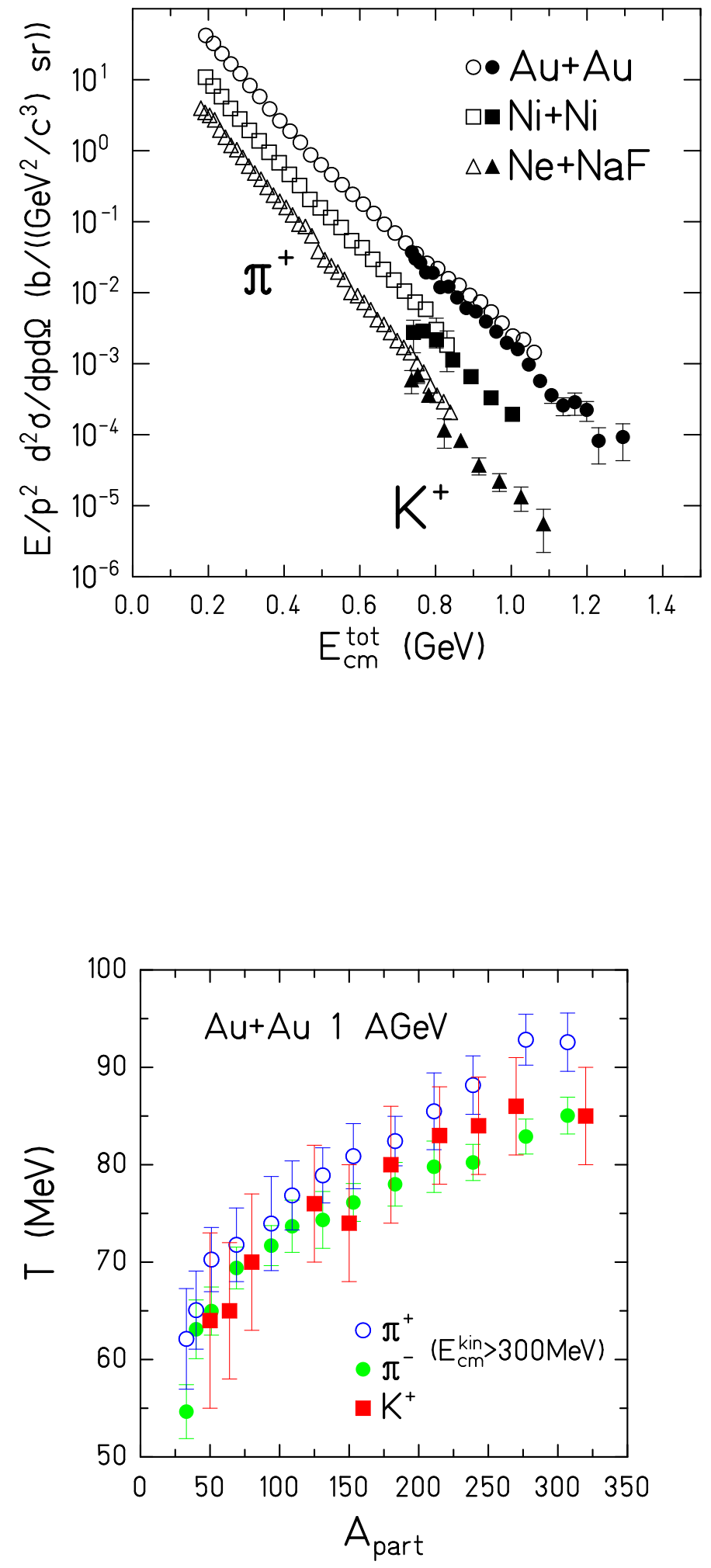

Figure 4.6: Invariant production cross sections for $\pi^{+}$(open symbols) and $\mathrm{K}^{+}$mesons (full symbols) as a function of the kinetic energy (plus the energy needed to produce the particle) in $\mathrm{Ne}+\mathrm{NaF}$, $\mathrm{Ni}+\mathrm{Ni}$ and $\mathrm{Au}+\mathrm{Au}$ collisions at $1 \mathrm{AGeV}$ (see text) 52, 111, 110.
Figure 4.7: Inverse slope parameter for high-energy pions $\left(\mathrm{E}_{c m}^{k i n}>300 \mathrm{MeV}\right)$ and $\mathrm{K}^{+}$ mesons in $\mathrm{Au}+\mathrm{Au}$ collisions at 1 $\mathrm{AGeV}$ (see text). The data are taken at $\Theta_{l a b}=44^{0}$ [73, 110]. 


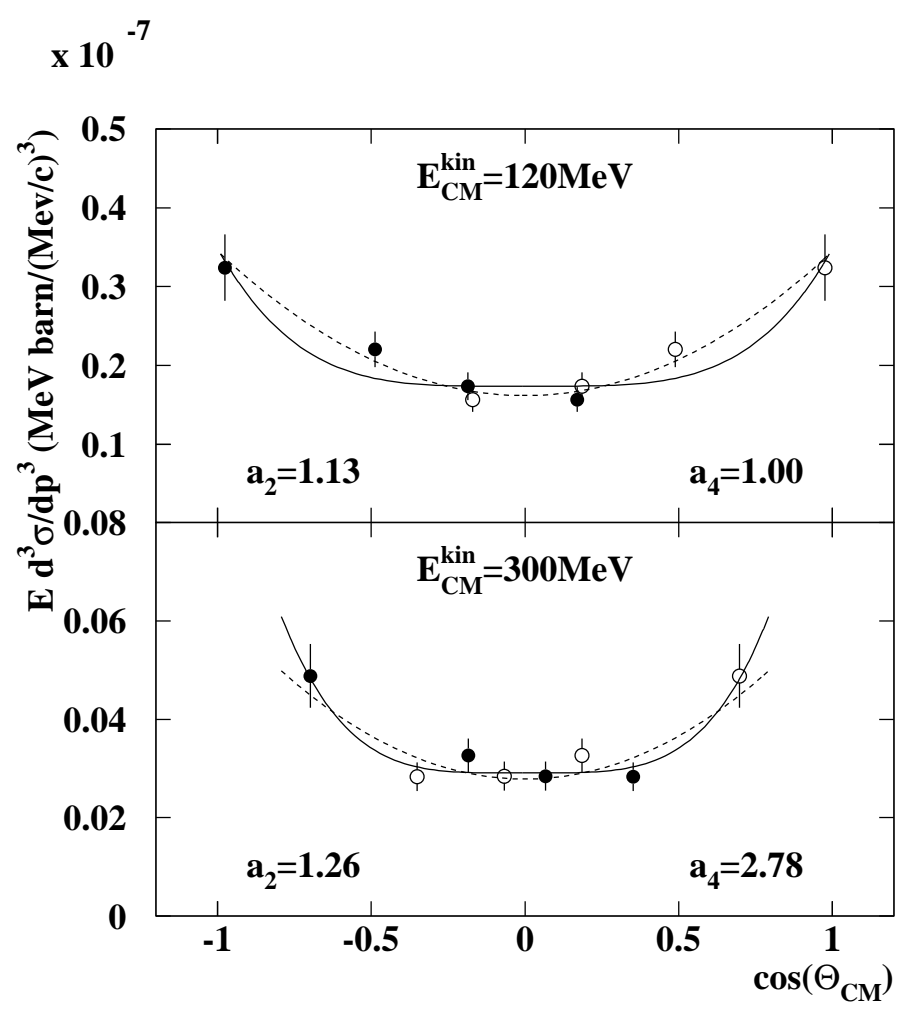

Figure 4.8: Invariant $\mathrm{K}^{+}$production cross section as a function of the c.m. polar emission angle in $\mathrm{Au}+\mathrm{Au}$ collisions at $1 \mathrm{AGeV}$ 110 for a kaon kinetic energy of 120 $\mathrm{MeV}$ (top) and $300 \mathrm{MeV}$ (bottom). The full symbols represent measured data, the open ones are reflected around 0 . The parameterization $\sigma_{i n v} \propto\left(1+a_{n} \cos ^{n} \Theta_{c m}\right)$ is fitted to the data with $\mathrm{n}=2$ (dashed lines) and $\mathrm{n}=4$ (solid lines).

high temperature phase [79, 141]. The large value of the slope parameter may be an artefact of the averaging over c.m-angle in reference [129] in the presence of a nonisotropic $\mathrm{K}^{+}$angular distribution. This observable will be discussed in the following section.

\section{3 $\mathrm{K}^{+}$angular distributions}

$\mathrm{K}^{+}$inclusive double differential cross sections were measured at $\Theta_{\text {lab }}=44^{\circ}$ with a magnetic spectrometer 142 and at $\Theta_{l a b}=85^{\circ}$ and $125^{\circ}$ with plastic-scintillator range telescopes [143 by the KaoS/CHIC Collaboration. The quoted $\mathrm{K}^{+}$yields at different angles are inconsistent with the assumption of an isotropic emission in the fireball system and were explained by an anisotropy of the polar angle distribution caused by $\mathrm{K}^{+}$rescattering $[143]$.

Recently $\mathrm{K}^{+}$production has been studied in $\mathrm{Au}+\mathrm{Au}$ collisions at $1 \mathrm{AGeV}$ as a function of impact parameter with a more complete phase space coverage. [110]. Fig. 4.8 presents the $\mathrm{K}^{+}$invariant cross section at $\mathrm{E}_{c m}^{k i n}=120 \mathrm{MeV}$ and $300 \mathrm{MeV}$ as a function of $\cos \Theta_{c m}$ for inclusive collisions. The data are parameterized by $\sigma_{i n v} \propto\left(1+a_{n} \cos ^{n} \Theta_{c m}\right)$ with $\mathrm{n}=2$ (dashed line) and $\mathrm{n}=4$ (solid line). The fitted parameters $a_{2}$ and $a_{4}$ are given in the figure. When taking into account the polar anisotropy (which is assumed to be independent of $\mathrm{E}_{c m}^{k i n}$ ) the total $\mathrm{K}^{+}$production cross section in $\mathrm{Au}+\mathrm{Au}$ collisions at $1 \mathrm{AGeV}$ is found to be $26 \pm 5 \mathrm{mb}$ (preliminary value). This value agrees (within the systematic errors) with the one obtained from the $\mathrm{K}^{+}$ data measured around midrapidity and extrapolated to $4 \pi$ assuming isotropic emission: $\sigma_{K^{+}}=24 \pm 5 \mathrm{mb}$.

Transport model calculations are in quantitive agreement with the measured $\mathrm{K}^{+}$polar angular distribution [125, 144]. These calculations claim that the $\mathrm{K}^{+}$forward-backward anisotropy is caused by (i) kaon-nucleon rescattering and (ii) by the alignment of pion-nucleon collisions along the beam axis which are the dominant source of kaons (via secondary interactions $\pi \mathrm{N} \rightarrow \mathrm{KYN}$ ).

Another important feature of kaon emission in nucleus-nucleus collisions is the azimuthal angular distribution which is affected by kaon propagation and hence by the kaon-nucleon interaction in the nuclear medium 123 . 


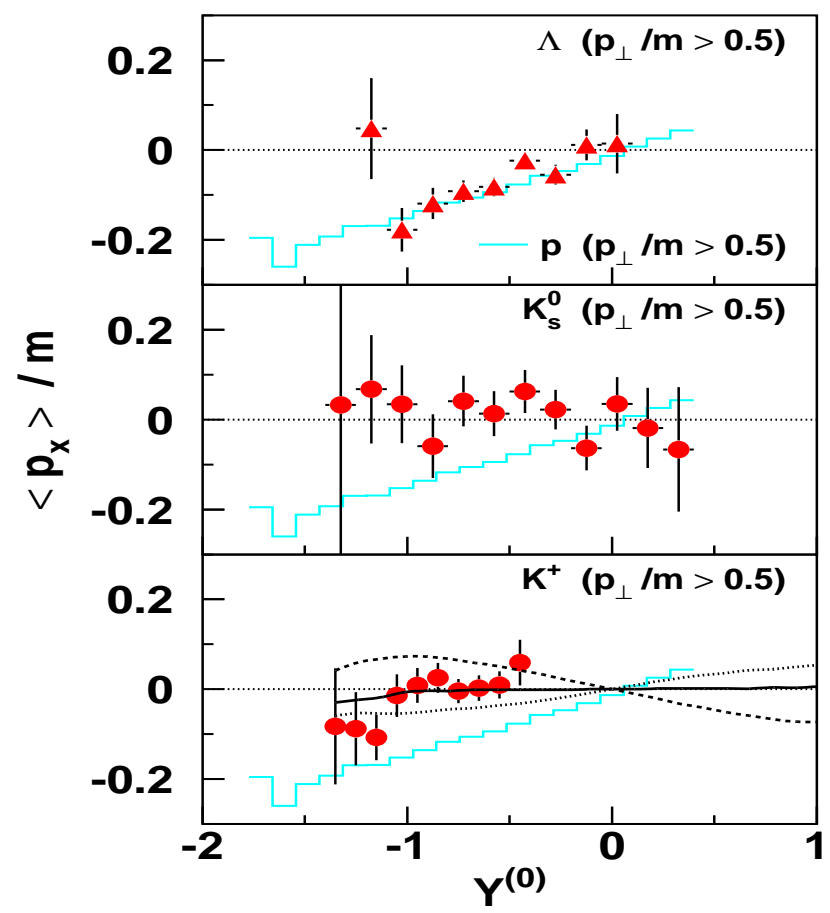

Figure 4.9: $\quad$ Sideward flow of strange particles [128. Average transverse momenta per mass projected onto the event plane as a function of the normalized rapidity for $\Lambda$ (top), $\mathrm{K}^{0}$ (middle) and $\mathrm{K}^{+}$(bottom). Protons are shown for comparison (diamonds and histogramms). The lines in the lower panel correspond to RBUU calculations $[126]$.

In section 3.3 we have discussed the azimuthally anisotropic emission of pions. Microscopic models explain this effect by rescattering and/or reabsorption in the spectator matter. In contrast, the enhanced emission of nucleons and light fragments in-plane ("sideward flow") and out-of plane ("squeeze-out") was explained by the hydrodynamical expansion of nuclear matter [2].

The in-plane flow of $\Lambda, \mathrm{K}_{s}^{0}$ and $\mathrm{K}^{+}$has been measured for $\mathrm{Ni}+\mathrm{Ni}$ collsions at $1.93 \mathrm{AGeV}$ by the FOPI Collaboration 128 . The results are shown in Fig. 4.9. No evidence for sidewards flow of kaons is seen whereas both the $\Lambda$ 's and protons exhibit a clear flow signal. The absence of kaon flow was interpreted as a signature for a weakly repulsive in-medium kaon-nucleon potential 126 . A more detailed discussion of in-medium effects on strange mesons is presented in the next subsection.

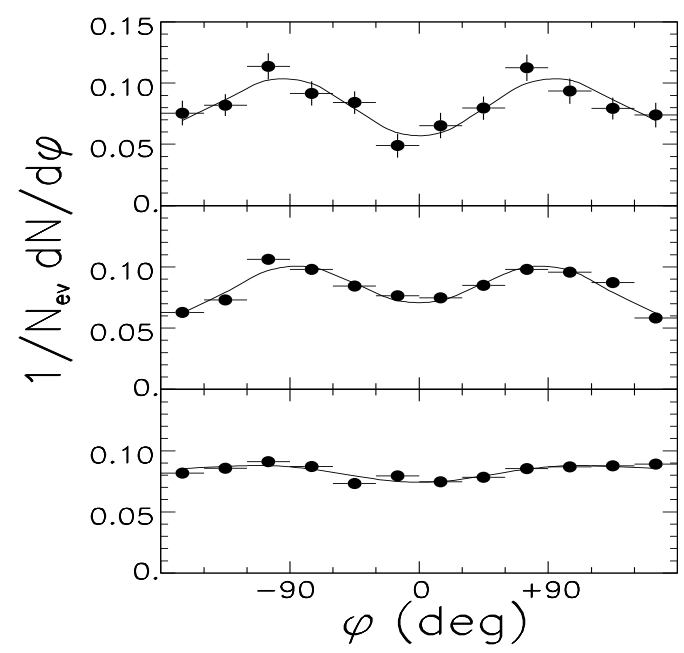

Figure 4.10: $\mathrm{K}^{+}$azimuthal angular distribution for peripheral $(b \geq 10 \mathrm{fm})$, semi-central ( $b=$ $5-10 \mathrm{fm})$ and central $(\mathrm{b} \leq 5$ fm) $\mathrm{Au}+\mathrm{Au}$ collisions at $1 \mathrm{AGeV}$ (from top to bottom). The data cover normalized rapidities in the interval $0.2 \leq y / y_{\text {proj }}<0.8$ and transverse momenta in the interval $0.2 \mathrm{GeV} / \mathrm{c} \leq p_{t}<0.8 \mathrm{GeV} / \mathrm{c}$. The lines represent fits to the data 145. (see text)

An anisotropic azimuthal emission pattern of $\mathrm{K}^{+}$mesons has been found recently by the KaoS Collaboration 145]. The kaons were measured in $\mathrm{Au}+\mathrm{Au}$ collisions at $1 \mathrm{AGeV}$ within the range of transverse 

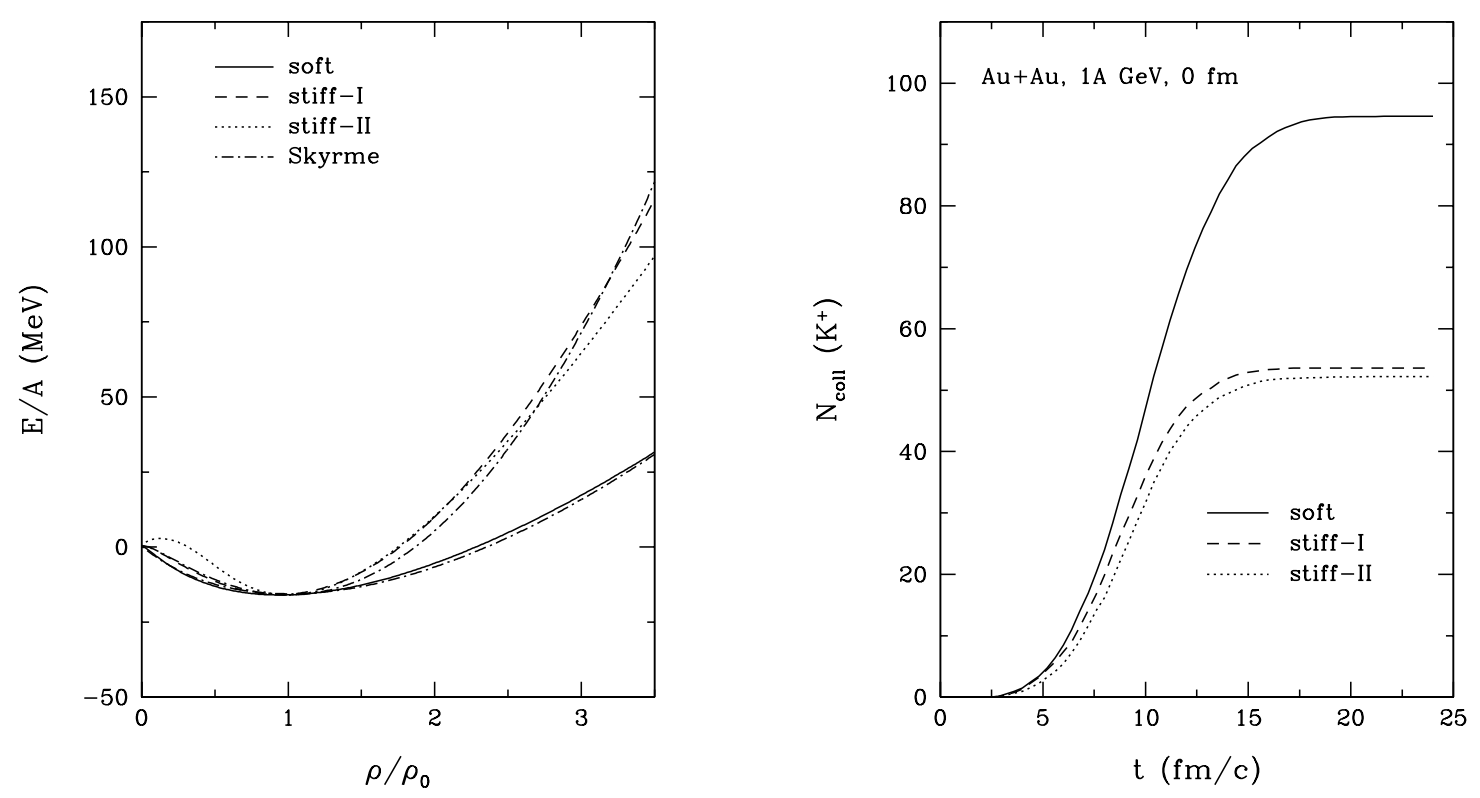

Figure 4.11: Left: The nuclear equation of state in the non-linear $\sigma-\omega$ model and in the Skyrme parameterization (taken from [119]). Right: The total number of baryon-baryon collisions that have energies above the kaon production threshold as a function of time for different equations of state [119]

momenta of $0.2<p_{T}<0.8 \mathrm{GeV} / \mathrm{c}$ and normalized rapidities of $0.2<y / y_{\text {proj }}<0.8$. Fig. 4.10 shows the azimuthal distribution $\mathrm{dN} / \mathrm{d} \phi$ as a function of the azimuthal emission angle $\phi$ for peripheral (top), semicentral (middle) and central collisions (bottom). The data are corrected for the dispersion of the reaction plane measurement [145]. The distributions peak at $\phi= \pm 90^{0}$ which corresponds to the directions perpendicular to the reaction plane. The anisotropy ratios (see equation 3.3 ) are $\mathrm{R}_{N}^{\text {corr }}=1.68 \pm 0.18,1.58 \pm 0.06$ and $1.09 \pm 0.03$ for peripheral, semi-central and central collisions, respectively.

If the anisotropy is caused by interaction of the kaons with the spectator matter the emission pattern provides a time scale for kaon emission: the $\mathrm{K}^{+}$mesons have to freeze-out within $15-20 \mathrm{fm} / \mathrm{c}$ after the first nuclear contact. This duration corresponds to the time which a spectator fragment needs to pass by the reaction zone at a beam energy of $1 \mathrm{AGeV}$. Transport calculations confirm this time span which coincides with the lifetime of the dense nuclear fireball: In $\mathrm{Au}+\mathrm{Au}$ collisions at $1 \mathrm{AGeV}$ the $\mathrm{K}^{+}$mesons are produced within the time interval of $5 \mathrm{fm} / \mathrm{c}<\mathrm{t}<17 \mathrm{fm} / \mathrm{c}$. During this period the baryonic density of the reaction zone is expected to be more than twice as high as the nuclear ground state density 123.

\subsection{Probing the nuclear equation of state}

Subthreshold $\mathrm{K}^{+}$production in relativistic nucleus-nucleus collisions is one of the most promissing probes to study the properties of nuclear matter at densities far from the ground state density. The sensitivity of kaon production on matter properties is based on (i) the collective production processes via multiple interactions which are strongly enhanced in the dense phase of the collision and (ii) the long mean free path of $\mathrm{K}^{+}$mesons. Early transport calculations (BUU) predicted for central heavy ion collisions at 0.7 $\mathrm{AGeV}$ that the $\mathrm{K}^{+}$yield obtained with a soft equation-of-state (EOS) is about 2-3 times higher than for a stiff EOS [117]. This sensitivity to the EOS is expected to diminish for smaller collision systems or beam energies above the kaon production threshold. These features have been reproduced recently by relativistic transport models (RBUU) [119].

Fig. 4.11 (left) displays the compressional energy per nucleon for different parameterizations as used 


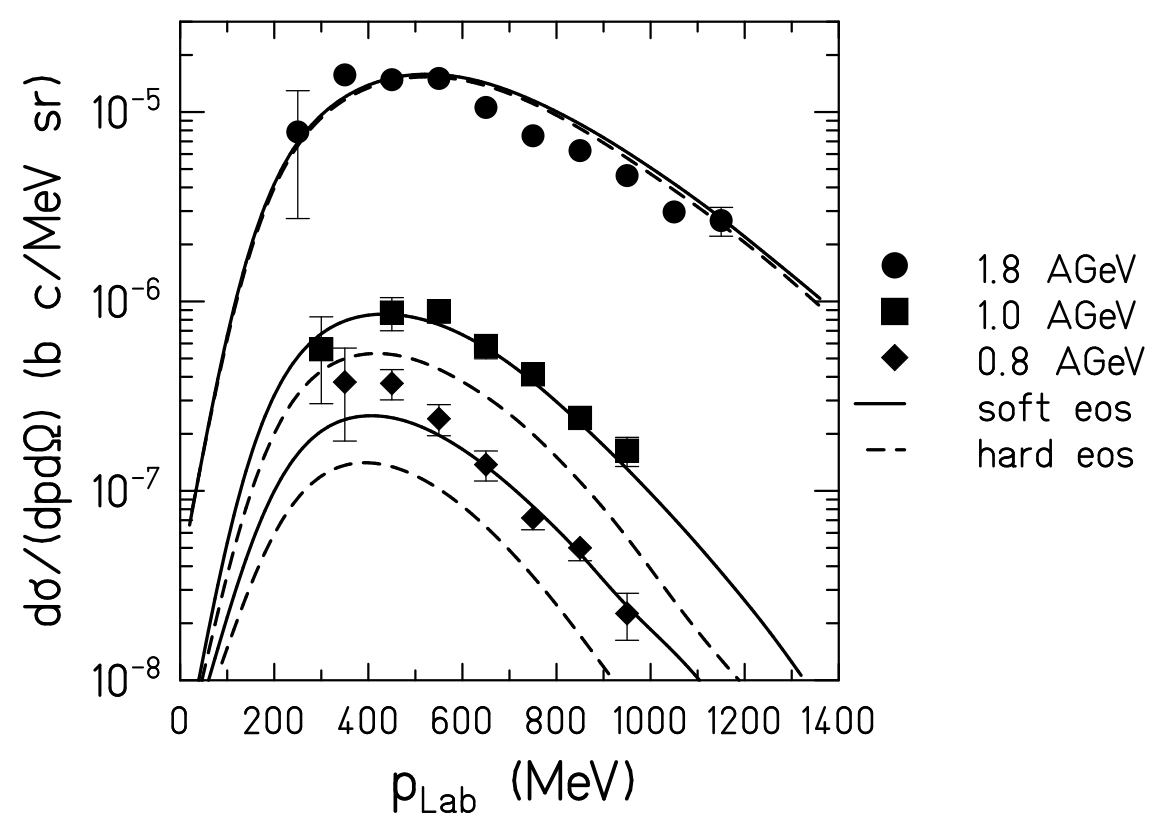

Figure 4.12: Double-differential $\mathrm{K}^{+}$production cross section measured in $\mathrm{Ni}+\mathrm{Ni}$ collisions $(0.8,1.0,1.8$ $\mathrm{AGeV})$ at $\Theta_{l a b}=44^{0}$ as a function of laboratory momentum [111. The lines represent results of QMD calculations for a soft (solid line) and a hard (dashed line) equation of state [135].

in the RBUU code. In the case of a soft EOS (solid line), much less kinetic energy is converted into compressional energy as compared to a stiff EOS (dashed line). Consequently, more thermal energy is available for particle production for a soft than for a stiff EOS. This is demonstrated in Fig. 4.11 (right) for central $\mathrm{Au}+\mathrm{Au}$ collisions at $1 \mathrm{AGeV}$. RBUU calculations find 95 baryon-baryon collisions with energies above the kaon production threshold when assuming a soft EOS but only 54 of those collisions for a stiff EOS. Forthermore, the average density at which kaons are produced is lower for a stiff EOS (about $2.1 \rho_{o}$ ) than for a soft EOS (about $2.5 \rho_{o}$ ) 119].

All transport models find consistently that the dominant channels contributing to $\mathrm{K}^{+}$production are $\pi \mathrm{N} \rightarrow \mathrm{KY}$ and $\Delta \mathrm{N} \rightarrow \mathrm{KYN}$ with $\mathrm{Y}=\Lambda, \Sigma[118,124,125$. The rate of these secondary collisions depends not only on the nuclear density and on the "thermal" energy of the baryons, but also on the abundance of resonances and pions and on their in-medium cross sections. An important input to these simulations are parameterizations of the elementary kaon production cross sections for the $\mathrm{NN}$ and $\Delta \mathrm{N}$ channels. So far the models used parameterizations for the cross section $\mathrm{NN} \rightarrow \mathrm{K}^{+} \mathrm{YN}$ which differ dramatically near threshold 112, 146, 147, 148. Recent data on inclusive $\mathrm{K}^{+}$production cross sections from pp collisions measured at COSY resolved the ambiguities [149]. New calculations which take into account a proper lifetime of the $\Delta$-resonance find that the $\pi \mathrm{N}$ channel is even more important than the $\Delta \mathrm{N}$ channel [118, 125].

The transport model calculations reproduce reasonably well the gross properties of the experimental $\mathrm{K}^{+}$data 123, 124, 135]. They are able to describe double-differential $\mathrm{K}^{+}$production cross sections as a function of bombarding energy and mass of the colliding nuclei over several orders of magnitude (within a factor of about 2). Unfortunately, the theoretical uncertainties on the absolute $\mathrm{K}^{+}$yields (resulting from the in-medium production cross sections) are still of the same size as the effect induced by the different equations of state. Nevertheless, some of the uncertainties can be reduced when comparing the results obtained for light and heavy collision systems or for different bombarding energies [116, 123, 135]. 


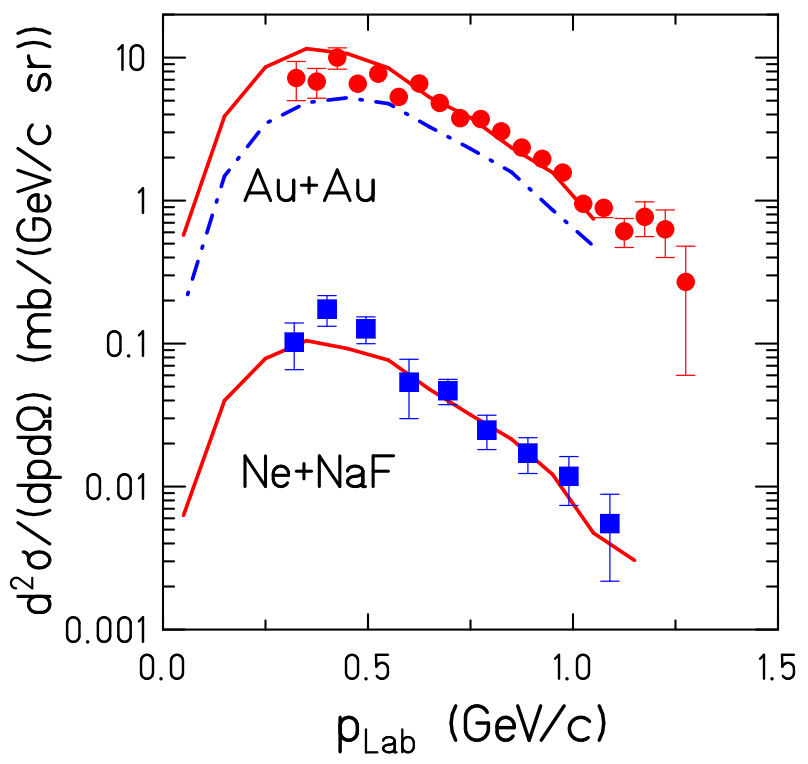

Figure 4.13: Double-differential $\mathrm{K}^{+}$production cross section measured at $1.0 \mathrm{AGeV}$ and $\Theta_{l a b}=44^{0}$ as a function of laboratory momentum (circles: $\mathrm{Au}+\mathrm{Au}$ [110], squares: $\mathrm{Ne}+\mathrm{NaF}$ [52]). The lines represent results of RBUU calculations for a soft (solid line) and a hard (dashed-dotted line) equation of state [123].

Such comparisons are shown in Fig. 4.12 and Fig. 4.13 which present calculated and measured doubledifferential cross sections for $\mathrm{K}^{+}$production measured at $\Theta_{l a b}=44^{\circ}$. Fig. 4.12 shows results for $\mathrm{Ni}+\mathrm{Ni}$ collisions at $0.8,1.0$ and $1.8 \mathrm{AGeV}[111]$. The data are compared with results from a QMD calculation [135] for a soft (solid lines) and a stiff equation-of-state (dashed lines). At a bombarding energy of 1.8 $\mathrm{AGeV}$ no effect of the EOS can be seen whereas at subthreshold beam energies the calculations differ by a factor of two depending on the EOS. The calculations use an old parameterization of the elementary $\mathrm{K}^{+}$production cross section according to Ref. [146] and neglect momentum-dependent interactions. Taking those into account would reduce the $\mathrm{K}^{+}$yield by a factor of about two. Furthermore, this calculation omits the reaction channel $\pi \mathrm{N} \rightarrow \mathrm{KY}$ as well as kaon rescattering and neglects the kaon-nucleon potential. These processes, however, will not depend strongly on the beam energy. The absolute agreement of model predictions with the data as shown in Fig. 4.12 is probably accidental and should not be considered as a support for the underlying model assumptions. Nevertheless the calculations demonstrate that when "normalizing" the theoretical $\mathrm{K}^{+}$spectrum to the data taken at $1.8 \mathrm{AGeV}$, the $\mathrm{K}^{+}$production cross sections measured at subthreshold beam energies support the assumption of a soft equation-of-state.

Fig. 4.13 shows experimental results for two different system sizes: $\mathrm{Ne}+\mathrm{NaF}$ and $\mathrm{Au}+\mathrm{Au}$ collisions at $1.0 \mathrm{AGeV}$ [22, 110]. The data are compared to relativistic transport calculations (RBUU) with a soft (solid lines) and stiff EOS (dashed line) [123, 119. For the light system $\mathrm{Ne}+\mathrm{NaF}$, the results for a stiff and a soft EOS coincide. The model takes into account the momentum dependence of the NN interaction, kaon final state interactions and an in-medium kaon-nucleon potential. However, the calculations use a parameterization of the $\mathrm{NN} \rightarrow \mathrm{KYN}$ process [112] which was found to give a too large cross section near threshold. Moreover, the process $\pi \mathrm{N} \rightarrow \mathrm{KY}$ is neglected. Therefore, the absolute agreement of model results and data as shown in Fig. 4.13 should not be overinterpreted. On the other hand, the theoretical uncertainties affect both the light and the heavy collision system in a similar way. Therefore, the relative agreement of the model calculation with both the $\mathrm{Ne}+\mathrm{NaF}$ and the $\mathrm{Au}+\mathrm{Au}$ data again favors a soft EOS.

As a summary of our discussion of this important aspect of nuclear physics we stress that according to transport models the $\mathrm{K}^{+}$abundances measured in central $\mathrm{Au}+\mathrm{Au}$ collisions at energies below the $\mathrm{p}+\mathrm{p} \rightarrow \mathrm{K}^{+}+\mathrm{X}$ threshold constrains the compressibility of nuclear matter at high density to values below 300 $\mathrm{MeV}$. This result is in agreement with calculations which explain simultaneously data on kaon production 
and on the directed flow of baryons in the same reaction [124]. However, estimates of the nuclear compressibility based on the suppressed pion production ask for a stiff eos [1]. We interprete this discrepancy as an incomplete understanding of pion production, propagation ans absorption in a dense nuclear medium (see also section 3.2).

\subsection{Kaons and antikaons in dense nuclear matter}

The formation of a nuclear fireball in central nucleus-nucleus collisions provides a possibility to study the properties of hadrons under extreme conditions. Inside the hot and dense nuclear medium, chiral symmetry (which is spontaneously broken in the vacuum) is expected to be partially restored and the properties of hadrons may change considerably [122]. A possible consequence of this effect is the modification of vector meson masses as studied in heavy ion collisions by dilepton experiments [150, 151.

The K-meson is another promising candidate for the experimental study of in-medium modifications. The properties of kaons and antikaons in dense nuclear matter have been investigated using chiral perturbation theory 120, 121, chiral dynamics 152, a relativistic mean field model 153 and a multi-channel approach [154]. The calculations find a KN potential which can be expressed by an attractive scalar part and a repulsive vector part. The attractive kaon-nucleon s-wave interaction is related to explicit chiral symmetry breaking by the large strange quark mass. The vector potential changes its sign for antikaons and is attractive for these particles. Therefore, the sum of the scalar and vector potential - which is slightly repulsive for kaons - becomes strongly attractive for antikaons. These results are consistent with an analysis of kaon-nucleon scattering data and kaonic atoms [155, 156. Fig. 4.14 shows the in-medium kaon and antikaon effective mass as calculated by different theoretical models as a function of nuclear density (taken from [157). A common feature of the different model calculations is that the kaon mass weakly increases whereas the antikaon mass considerably decreases with increasing nuclear density. The latter effect may lead to $\mathrm{K}^{-}$condensation in neutron stars $[158,159,160$ and possibly even to the formation of low mass black holes in the galaxy 161, 162, 163.

The existence of a KN potential affects both the production and the propagation of kaons and antikaons in nuclear matter. A weakly repulsive $\mathrm{K}^{+} \mathrm{N}$ interaction should repel the $\mathrm{K}^{+}$mesons from the nucleons. This effect is expected to modify the $\mathrm{K}^{+}$azimuthal emission pattern in such a way that it differs from the proton azimuthal distribution $\left[126\right.$. First experimental results on the $\mathrm{K}^{+}$and $\Lambda$ directed flow were reported by the FOPI Collaboration $[128]$. Fig. 4.9 shows the average transverse velocity projected onto the reaction plane $<p_{x}>/ \mathrm{m}$ as a function of the normalized c.m. rapidity for $\Lambda$ (top), $\mathrm{K}_{s}^{o}$ (middle) and $\mathrm{K}^{+}$ (bottom) in comparison to protons (histogram). The $\Lambda^{\prime}$ 's exhibit the same flow behaviour as the protons whereas the kaons do not show any significant flow signal. The $\mathrm{K}^{+}$data are compared to results of RBUU calculations assuming different in-medium kaon potentials: no potential (dotted line), vector potential only (dashed line) and scalar + vector potential (solid line) [126. The data seem to favor the assumption of a weakly repulsive in-medium kaon potential.

According to transport calculations, the existence of a strong in-medium kaon potential affects also the $\mathrm{K}^{+}$azimuthal angular distribution at midrapidity. The experimental results are shown in Fig. 4.15 for semi-central $\mathrm{Au}+\mathrm{Au}$ collisions at $1 \mathrm{AGeV} 145$. The data have been taken in a small interval of normalized rapidity $0.4<y / y_{\text {proj }}<0.6$. The solid line corresponds to a RBUU calculation which includes a strong in-medium KN potential whereas the dashed line is the result without an in-medium KN potential [127]. The strong KN potential is clearly needed to reproduce the data.

Due to the strongly attractive mean-field potential of antikaons, the azimuthal emission pattern of $\mathrm{K}^{-}$ mesons is expected to be modified dramatically and therefore should provide an unique signature of the in-medium effect 164. The strong absorption of $\mathrm{K}^{-}$mesons - which would lead to a pronounced $\mathrm{K}^{-}$ antiflow pattern near target rapidity - is predicted to be cancelled by the strongly attractive in-medium antikaon-nucleon interaction. The resulting $\mathrm{K}^{-}$azimuthal distribution at backward rapidities is expected to be rather flat in contrast to the one of protons - if the KN potential exists. Up to now no data on the $\mathrm{K}^{-}$azimuthal emission pattern are available. Experiments on this issue are scheduled at SIS. 


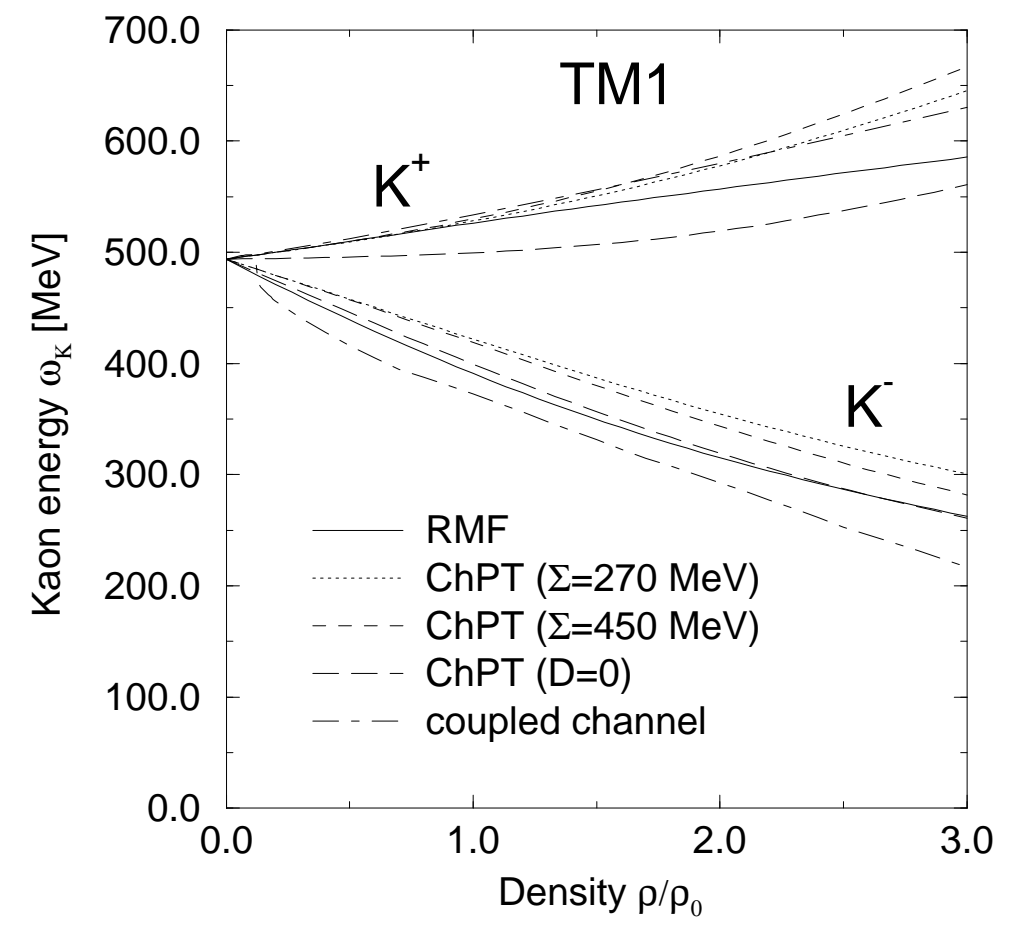

Figure 4.14: The energy of kaons and antikaons in nuclear matter as a function of density for a soft equation of state (taken from [157.

The modifications of the masses of kaons and antikaons in dense nuclear matter will affect their yields differently. Therefore, the cross section ratio of kaons and antikaons is a sensitive probe of in-medium KN potentials [165, 124, 125]. As shown in Fig. 4.14, the antikaon effective mass is predicted to drop significantly in dense nuclear matter while the $\mathrm{K}^{+}$effective mass increases weakly. This in-medium mass modification lowers the threshold for the antikaon production process $\mathrm{NN} \rightarrow \mathrm{K}^{+} \mathrm{K}^{-} \mathrm{NN}$ but increases it for kaon production via $\mathrm{NN} \rightarrow \mathrm{K}^{+} \mathrm{YN}$. Consequently the $\mathrm{K}^{+}$production is expected to be slightly suppressed whereas the $\mathrm{K}^{-}$production is strongly enhanced as compared to free $\mathrm{NN}$ collisions. The latter effect should be very pronounced at subthreshold bombarding energies, where the $\mathrm{K}^{-}$excitation function is very steep and thus acts as an amplifier: even a small mass reduction might result in a strong $\mathrm{K}^{-}$enhancement. The in-medium reduction of the threshold for the production of a $\mathrm{K}^{-}+\mathrm{K}^{+}$pair is due to the attractive $\mathrm{KN}$ potential only, as the vector parts of $\mathrm{K}^{+}$and $\mathrm{K}^{-}$cancel.

The available data on subthreshold $\mathrm{K}^{-}$production in nucleus-nucleus collisions have been presented in section 4.1. The large $\mathrm{K}^{-}$yield measured in $\mathrm{Ni}+\mathrm{Ni}$ collisions at $1.85 \mathrm{AGeV}$ by the FRS group [165, 138] was reproduced by transport calculations (RBUU) if an in-medium mass reduction of the $\mathrm{K}^{-}$meson is assumed 165. The KaoS Collaboration found evidence for an enhanced $\mathrm{K}^{-}$yield in $\mathrm{Ni}+\mathrm{Ni}$ collisions from a comparison to the $\mathrm{K}^{+}$yield at equivalent beam energies 111]. Fig. 4.16 shows the invariant $\mathrm{K}^{+}$ production cross section (open symbols) as a function of the $\mathrm{K}^{+}$kinetic c.m. energy measured in $\mathrm{Ni}+\mathrm{Ni}$ collisions at $0.8,1.0$ and $1.8 \mathrm{AGeV}$ around midrapidity. The $\mathrm{K}^{-}$data (full symbols) measured at $1.8 \mathrm{AGeV}$ agree with the $\mathrm{K}^{-}$invariant cross section as measured in $\mathrm{Ni}+\mathrm{Ni}$ collisions at $1.85 \mathrm{AGeV}$ by the FRS at $\Theta_{l a b}=0^{\circ}$ [55, 137, 138].

The question is, whether there is experimental evidence for an enhancement of the $\mathrm{K}^{-}$yield measured at $1.8 \mathrm{AGeV}$ and how to quantify it. The KaoS Collaboration used the $\mathrm{K}^{+}$cross section mesured at 1.0 $\mathrm{AGeV}$ as a reference for $\mathrm{K}^{-}$production at $1.8 \mathrm{AGeV}$. These two beam energies are "equivalent" in the sense that they allow to study $\mathrm{K}^{+}$and $\mathrm{K}^{-}$production at the same Q-value [11]:

$$
\begin{aligned}
& \mathrm{Q}\left(\mathrm{NN} \rightarrow \mathrm{K}^{+} \Lambda \mathrm{N}\right)=\sqrt{s}-\sqrt{s}_{\text {thres }}=2.32 \mathrm{GeV}-2.55 \mathrm{GeV}=-0.23 \mathrm{GeV} \\
& \mathrm{Q}\left(\mathrm{NN} \rightarrow \mathrm{K}^{+} \mathrm{K}^{-} \mathrm{NN}\right)=\sqrt{s}-\sqrt{s}_{\text {thres }}=2.63 \mathrm{GeV}-2.86 \mathrm{GeV}=-0.23 \mathrm{GeV}
\end{aligned}
$$

The choice of the equivalent energies for the comparison of $\mathrm{K}^{+}$and $\mathrm{K}^{-}$production is meant to be a 


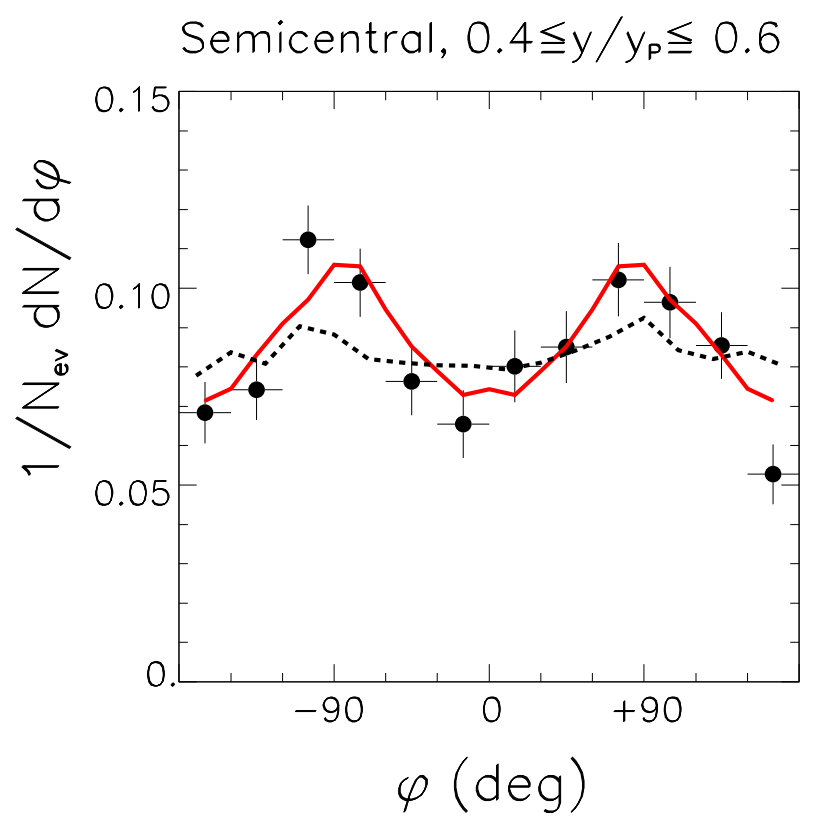

Figure 4.15: $\mathrm{K}^{+}$azimuthal distribution for semi-central $\mathrm{Au}+\mathrm{Au}$ collisions at $1 \mathrm{AGeV}$. The kaons have transverse momenta of $0.2 \mathrm{GeV} / \mathrm{c}<$ $p_{T}<0.8 \mathrm{GeV} / \mathrm{c}$ and are measured in the range of normalized rapidities of $0.4<y / y_{\text {proj }}<0.6$ 145. The lines represent results of RBUUcalculations with $\mathrm{K}^{+}$rescattering (dotted line) and with $\mathrm{K}^{+}$rescattering and an in-medium $\mathrm{KN}$ potential (solid line) [127.

crude correction for the differences in accessible phase space. According to Fig. 4.16 the $\mathrm{K}^{+}$yield at 1 $\mathrm{GeV} /$ nucleon agrees roughly with the $\mathrm{K}^{-}$yield at $1.8 \mathrm{GeV} /$ nucleon. Not only the cross section but also the dependence on $\mathrm{A}_{\text {part }}$ is the same for $\mathrm{K}^{+}$and $\mathrm{K}^{-}$observed at equivalent energies (see Fig. 4.4). This result for nucleus-nucleus collisions is quite different from the $\mathrm{K}^{-} / \mathrm{K}^{+}$ratio for proton-proton collisions at equivalent beam energies. Fig. 4.17 shows the available data (including the most recent results from COSY) on inclusive cross sections for $\mathrm{K}^{+}$and antikaon production in proton-proton collisions as a function of the energy above threshold [149, 166, 167]. The $\mathrm{K}^{+}$data exceed the antikaon data by 1-2 orders of magnitude. The lines represent a recent parameterization of the elementary kaon production cross sections 147, 168].

Before concluding on nontrivial in-medium effects one should check whether or not pion induced kaon production $\left(\pi \mathrm{N} \rightarrow \mathrm{K}^{+} \mathrm{Y}, \pi \mathrm{N} \rightarrow \mathrm{K}^{+} \mathrm{K}^{-} \mathrm{N}\right)$ causes an enhancement of the $\mathrm{K}^{-}$yield with respect to the $\mathrm{K}^{+}$ yield at equivalent beam energies. Typical values for the cross sections at the same energy above threshold are $\sigma\left(\pi^{+} \mathrm{p} \rightarrow \mathrm{K}^{+} \mathrm{Y}\right) \approx 300 \mu \mathrm{b}$ and $\sigma\left(\pi^{-} \mathrm{p} \rightarrow \mathrm{K}^{-} \mathrm{K}^{+} \mathrm{n}\right) \approx 200 \mu \mathrm{b}$ 115, 168, 169, 170, 171. The pion momentum thresholds are $900 \mathrm{MeV} / \mathrm{c}$ and $1500 \mathrm{MeV} / \mathrm{c}$ for the production of $\mathrm{K}^{+} \mathrm{Y}$ and $\mathrm{K}^{-} \mathrm{K}^{+}$pairs, respectively, on stationary protons. Assuming a mean momentum of $400 \mathrm{MeV} / \mathrm{c}$ for the nucleons (in the c.m. system) the relevant pion momenta are $600 \mathrm{MeV} / \mathrm{c}$ and $1000 \mathrm{MeV} / \mathrm{c}$.

From the pion spectra measured in $\mathrm{Ni}+\mathrm{Ni}$ collisions one can estimate that for a beam energy of $1 \mathrm{AGeV}$ the pion yield in the c.m. system above $600 \mathrm{MeV} / \mathrm{c}$ is about 10 times higher than the pion yield above $1000 \mathrm{MeV} / \mathrm{c}$ obtained for a beam energy of $1.8 \mathrm{AGeV}$ [73]. According to these numbers, the pion induced $\mathrm{K}^{-}$yield from $\mathrm{Ni}+\mathrm{Ni}$ collisions at $1.8 \mathrm{AGeV}$ is expected to be a factor of about 15 smaller than the pion induced $\mathrm{K}^{+}$yield from $\mathrm{Ni}+\mathrm{Ni}$ collisions at $1.0 \mathrm{AGeV}$. Therefore, it is very unlikely that pion induced kaon production accounts for the observed $\mathrm{K}^{-} / \mathrm{K}^{+}$ratio in $\mathrm{Ni}+\mathrm{Ni}$ collisions at equivalent energies. The above estimation is roughly confirmed by RBUU calculations which predict that for $\mathrm{Ni}+\mathrm{Ni}$ at $1.8 \mathrm{AGeV}$ pionbaryon collisions contribute only $5-10 \%$ to the $\mathrm{K}^{-}$yield (including $\mathrm{K}^{-}$absorption but without in-medium effects, see Fig.4.18) whereas more than $90 \%$ of the $\mathrm{K}^{+}$yield in $\mathrm{Ni}+\mathrm{Ni}$ at $1.0 \mathrm{AGeV}$ is due to $\pi \mathrm{N}$ and $\Delta \mathrm{N}$ collisions 124, 125.

From the above arguments we conclude, that the $\mathrm{K}^{-} / \mathrm{K}^{+}$ratio found in $\mathrm{Ni}+\mathrm{Ni}$ collisions at equivalent energies cannot be understood in terms of a superposition of free baryon-baryon or pion-baryon collisions producing the kaons. The large $\mathrm{K}^{-}$yield is even more surprising as $\mathrm{K}^{-}$mesons are strongly absorbed 

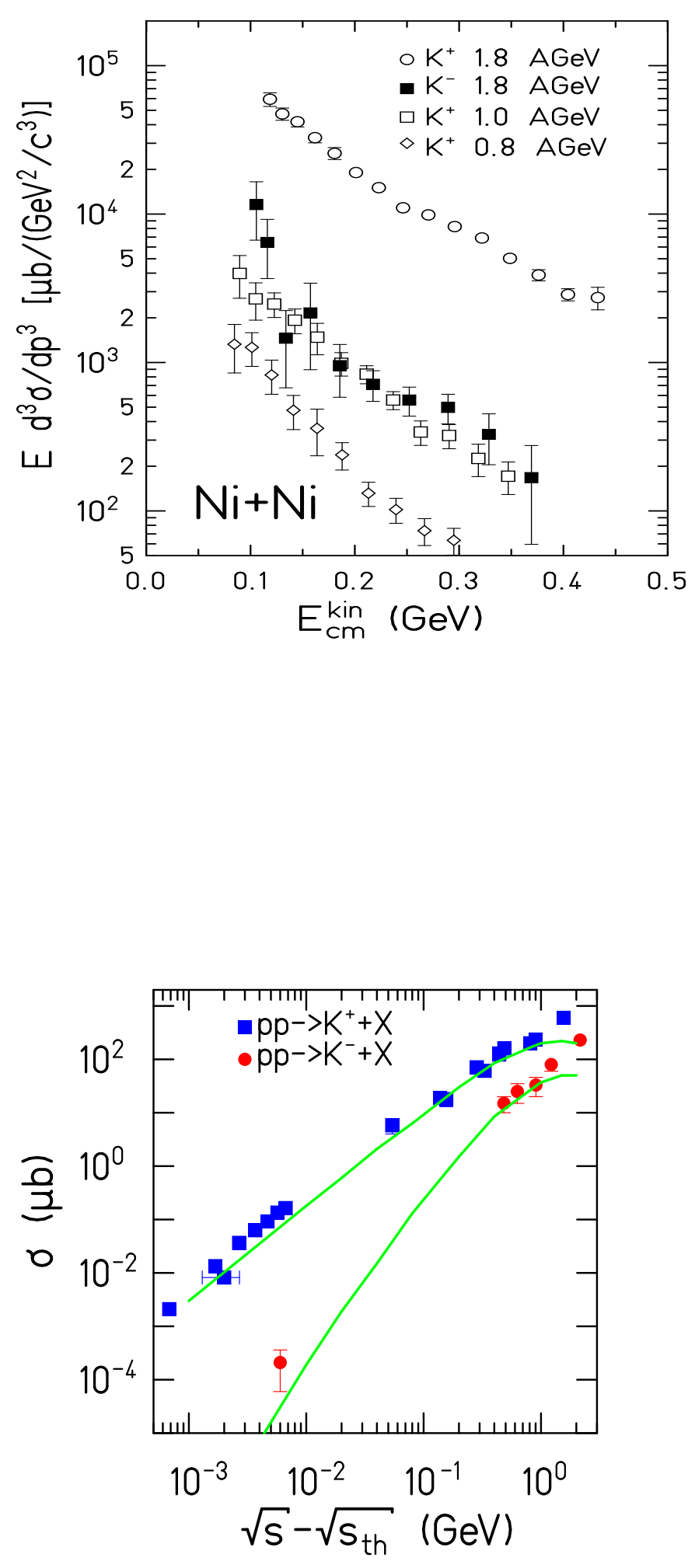

Figure 4.16: Inclusive invariant $\mathrm{K}$-meson production cross section measured in $\mathrm{Ni}+\mathrm{Ni}$ collisions at $\Theta_{l a b}=44^{0}$ 111. Open symbols: $\mathrm{K}^{+}(0.8,1.0,1.8 \mathrm{AGeV})$, full symbols: $\mathrm{K}^{-}(1.8 \mathrm{AGeV})$.
Figure 4.17: Kaon and antikaon production cross section measured in $\mathrm{p}+\mathrm{p}$ collisions as a function of the energy above threshold (Q-value). The data are taken from 149, 166, 167. The lines represent parameterizations 147, 168. 


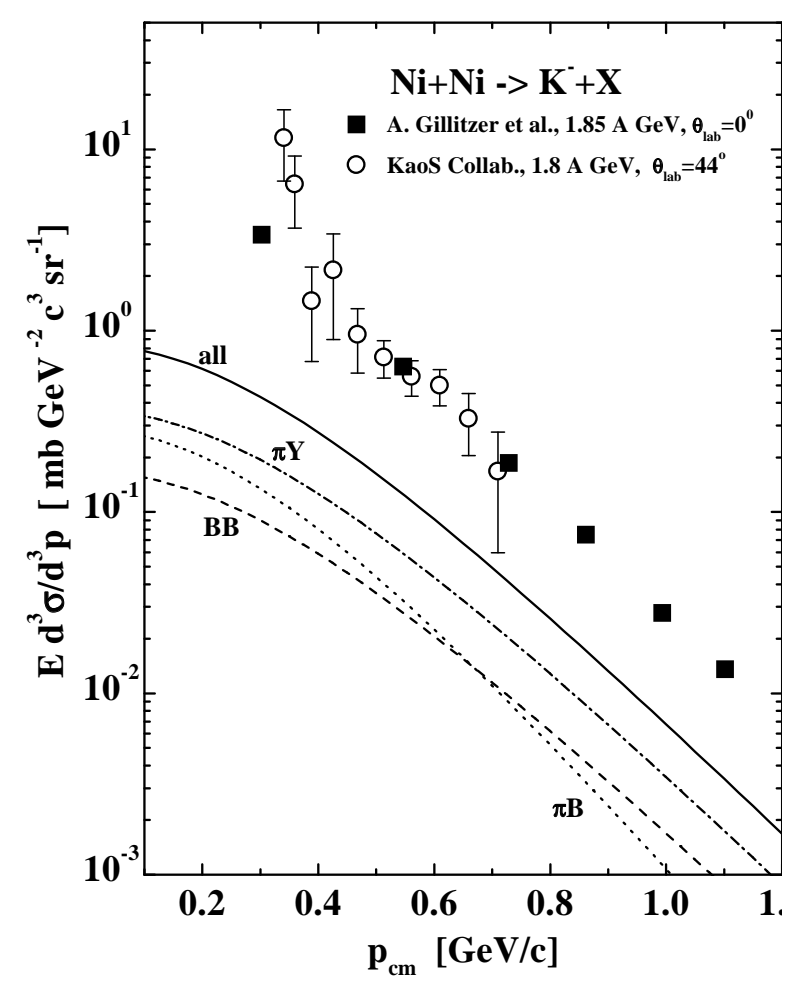

Figure 4.18: Inclusive invariant $\mathrm{K}^{-}$production cross section for $\mathrm{Ni}+\mathrm{Ni}$ collisions as a function of the c.m. momentum measured at $1.85 \mathrm{AGeV}$ and $\Theta_{l a b}=0^{0}$ (full squares 138]) and at $1.8 \mathrm{AGeV}$ and $\Theta_{l a b}=44^{\circ}$ (open circles [111]). The lines represent results of a RBUU calculation [124] without including in-medium effects (bare $\mathrm{K}^{-}$mass). The partial $\mathrm{K}^{-}$production channels are baryonbaryon (BB, dashed line), pion-baryon ( $\pi \mathrm{B}$, dotted line) and pion-hyperon $(\pi \mathrm{Y}$, dashed-dotted line) collisions. The solid line is the sum of all channels.

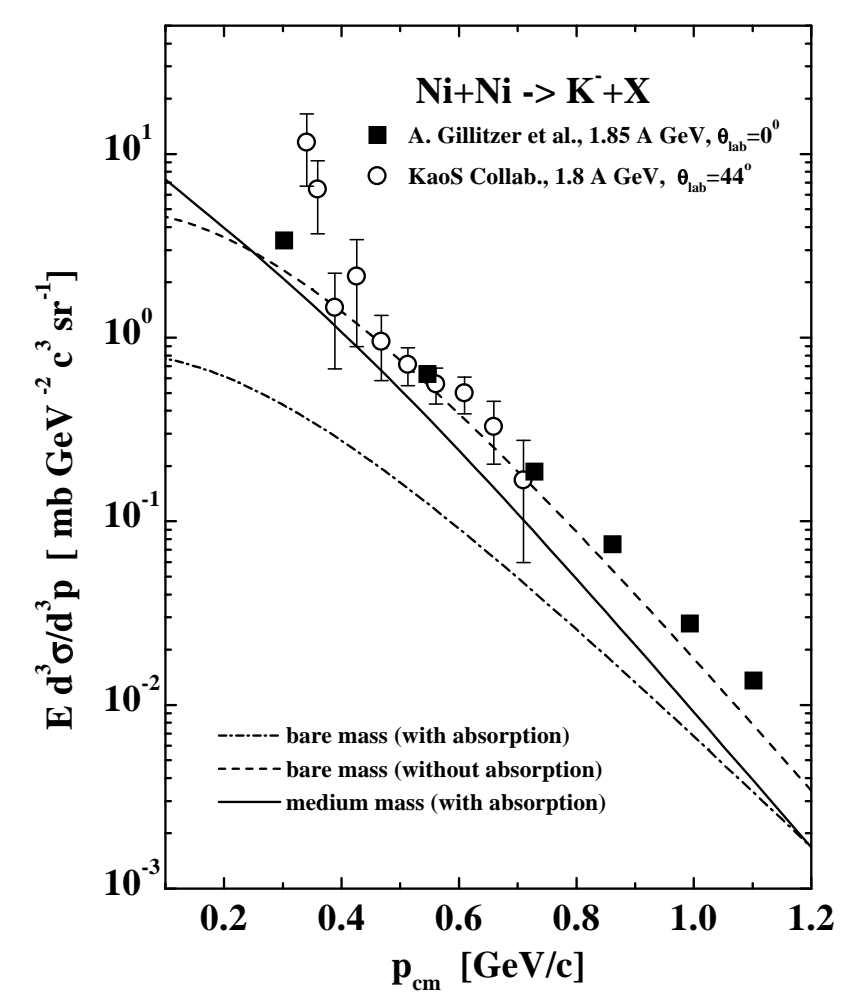

Figure 4.19: Inclusive invariant $\mathrm{K}^{-}$production cross section for $\mathrm{Ni}+\mathrm{Ni}$ collisions as a function of the c.m. momentum measured at $1.85 \mathrm{AGeV}$ and $\Theta_{l a b}=0^{0}$ (full squares 138) and at $1.8 \mathrm{AGeV}$ and $\Theta_{l a b}=44^{\circ}$ (open circles [111). The lines represent results of a RBUU calculation 124: bare $\mathrm{K}^{-}$mass with absorption (dashed-dotted), bare $\mathrm{K}^{-}$mass without absorption (dashed) and inmedium $\mathrm{K}^{-}$mass with absorption (solid, $\alpha=$ $0.2)$. 


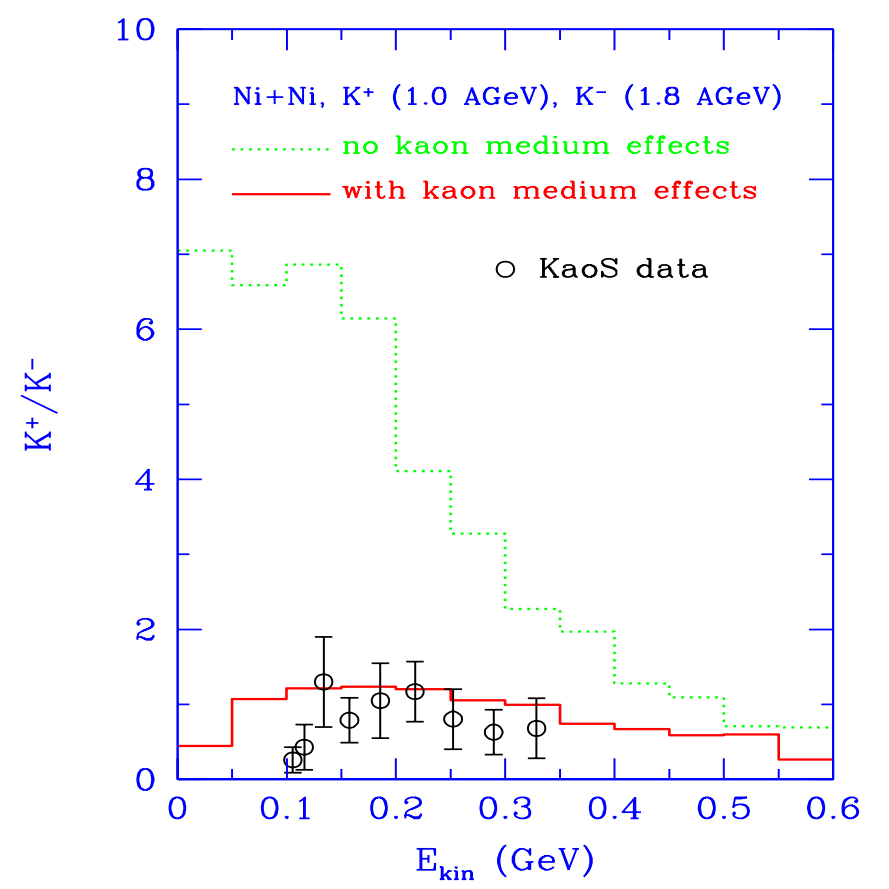

Figure 4.20: $\mathrm{K}^{+} / \mathrm{K}^{-}$ratio as a function of c.m. kinetic energy for $\mathrm{Ni}+\mathrm{Ni}$ collisions at equivalent beam energies $\left(\mathrm{K}^{+}\right.$measured at $1.0 \mathrm{AGeV}$ and $\mathrm{K}^{-}$at $1.8 \mathrm{AGeV}, \Theta_{l a b}=44^{0}$ ) 111. The lines show results of a RBUU calculation without (dotted) and with medium effects (solid) 162. 163.

in the nuclear medium by strangeness exchange reactions like $\mathrm{K}^{-} \mathrm{N} \rightarrow \mathrm{Y} \pi$ with $\mathrm{Y}=\Lambda, \Sigma$ [108, whereas the $\mathrm{K}^{+}$meson can hardly be absorbed due to its anti-strange quark content and the charge exchange reaction $\mathrm{K}^{+} \mathrm{n} \leftrightarrow \mathrm{K}^{0} \mathrm{p}$ does not lead to $\mathrm{K}^{+}$losses in isospin symmetric nuclear matter. On the other hand, the strangeness exchange reaction $\pi \mathrm{Y} \rightarrow \mathrm{K}^{-} \mathrm{N}$, which is the "inverse" of $\mathrm{K}^{-}$absorption, might be an additional source of in-medium $\mathrm{K}^{-}$production 172]. Indeed, RBUU calculations claim that (for bare kaon masses, see Fig. 4.18) the process $\pi \mathrm{Y} \rightarrow \mathrm{K}^{-} \mathrm{N}$ is the most important $\mathrm{K}^{-}$production channel in $\mathrm{Ni}+\mathrm{Ni}$ collisions at $1.8 \mathrm{AGeV}[124]$. This calculations is based on the parameterization of the elementary kaon production cross sections as shown in Fig. 4.17. Nevertheless, the sum of all $\mathrm{K}^{-}$production channels considered by the transport calculations is about a factor of 4-5 below the measured data points, if in-medium effects on the $\mathrm{K}^{-}$mass are omitted (see Fig. 4.18). In contrast, Fig. 4.19 demonstrates that reasonable agreement with the experimental data is achieved if an in-medium reduction of the $\mathrm{K}^{-}$mass is assumed according to $\mathrm{m}_{K}^{*}(\rho)=\mathrm{m}_{K}^{o}\left(1-\alpha \rho / \rho_{o}\right)$ with $\alpha=0.24$ [173]. As demonstrated in Fig. 4.14, various calculations of the kaon selfenergy in nuclear matter find similar reductions of the in-medium $\mathrm{K}^{-}$mass with increasing nuclear density.

The kaon in-medium modification and its effect on the kaon yields from $\mathrm{Ni}+\mathrm{Ni}$ collisions was also studied by the Stony Brook group using a RBUU transport code 162, 163]. The result of the calculation is presented in fig. 4.20. It shows the $\mathrm{K}^{+} / \mathrm{K}^{-}$ratio for equivalent beam energies as a function of kaon energy without (dotted line) and with kaon medium effects (solid line). In order to get agreement with the KaoS data (symbols) the authors varied the density dependence of the kaon potentials. Based on the kaon in-medium properties as constrained by the heavy ion data the authors found that the critical density for $\mathrm{K}^{-}$condensation is about $3 \rho_{o}$. This effect is predicted to limit the maximum possible mass of neutron stars to about 1.5 solar masses 162, 163.

\subsection{Strange meson production at ultrarelativistic energies}

Even at ultrarelativistic beam energies, the information about strange particle production is to a large extend contained in the $K$ - and $\bar{K}$-mesons. Only the strange quarks carried by the hyperon-antihyperon 
pairs are not accounted for. The number of strange quarks in those hyperons which originate from associated production can be inferred from the difference in number between $K$ and $\bar{K}$ (e.g. at high energy $N_{\text {hyp }}=$ $2.0 \cdot\left(N_{K^{+}}-N_{K^{-}}\right)$for isospin symmetric systems with the factor 2.0 accounting for $\left(N_{K^{0}}-N_{\overline{K^{0}}}\right)$ ).

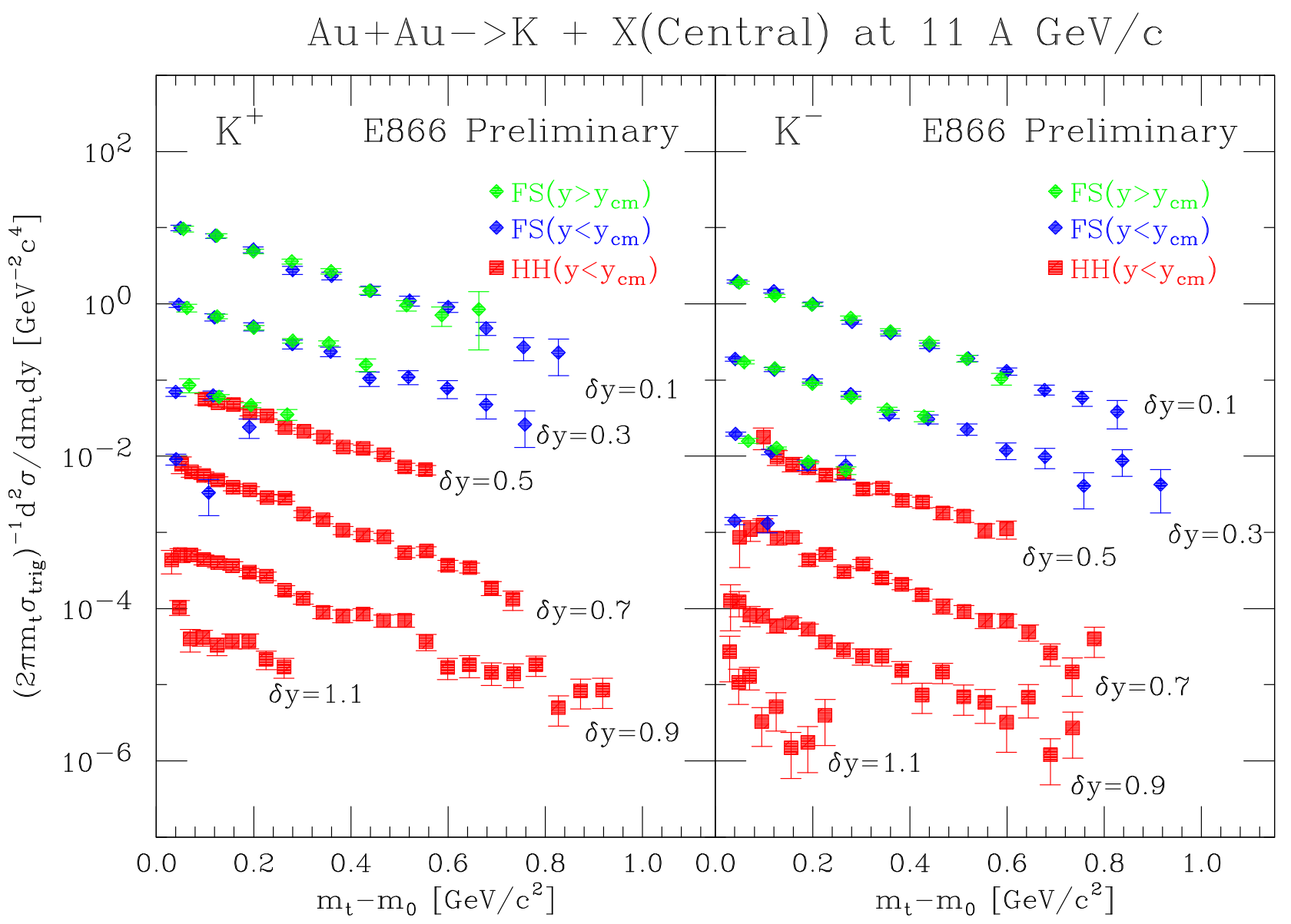

Figure 4.21: The $\mathrm{m}_{T}$ distributions of $\mathrm{K}^{+}$and $\mathrm{K}^{-}$mesons from central $\mathrm{Au}+\mathrm{Au}$ collisions at $10 \mathrm{AGeV}$ in different rapidity bins $\left(\delta \mathrm{y}=\left|\mathrm{y}-\mathrm{y}_{c m}\right|\right)$. Starting with $\delta \mathrm{y}=0.1$ at the top, each successive spectrum is divided by 10 [24].

The rapidity distribution of K-mesons have already been dealt with in chapter 2. It was shown that for A+A collisions at AGS and SPS energies the kaon rapidity distributions are significantly wider than expected for a thermal source.

Charged kaon spectra have been measured at the AGS for the (nearly) symmetric systems $\mathrm{Si}+\mathrm{Al}$ and $\mathrm{Au}+\mathrm{Au}$ in both peripheral and central collisions. Fig. 4.21 presents $\mathrm{m}_{T}$ distributions of $\mathrm{K}^{ \pm}$mesons from central $\mathrm{Au}+\mathrm{Au}$ collisions at $10 \mathrm{AGeV}$ in different rapidity bins [28, 24]. The spectra are well described by a single exponential and the inverse slope parameters are $170-200 \mathrm{MeV}$ both for $\mathrm{K}^{+}$and $\mathrm{K}^{-}$. These values are similar to the ones obtained in $\mathrm{Si}+\mathrm{Au}$ collisions. Fig. 4.22 shows $\mathrm{K}^{+}$and $\mathrm{K}^{-}$transverse mass spectra for very small values of $\mathrm{m}_{T}$ for the same collision system $[26]$. In the range of $\mathrm{m}_{T}-\mathrm{m}_{K}<0.1$, the inverse slope parameter has values of 60 to $90 \mathrm{MeV}$. The two data sets overlap in rapidity at $\mathrm{y}=2.4$. Both experimental results are still preliminary. It will be interesting to see whether the significant differences in slope parameters at small and large $\mathrm{m}_{T}$ persist. If so, a kaon low- $\mathrm{p}_{T}$ enhancement would emerge.

The most remarkable result is a "strangeness enhancement" seen in the $\mathrm{K}^{+} / \pi^{+}$ratio [24, 174]. Fig. 4.23 shows particle ratios measured at mid-rapidity as a function of centrality in $\mathrm{Au}+\mathrm{Au}$ collisions at $10.7 \mathrm{AGeV}$ 


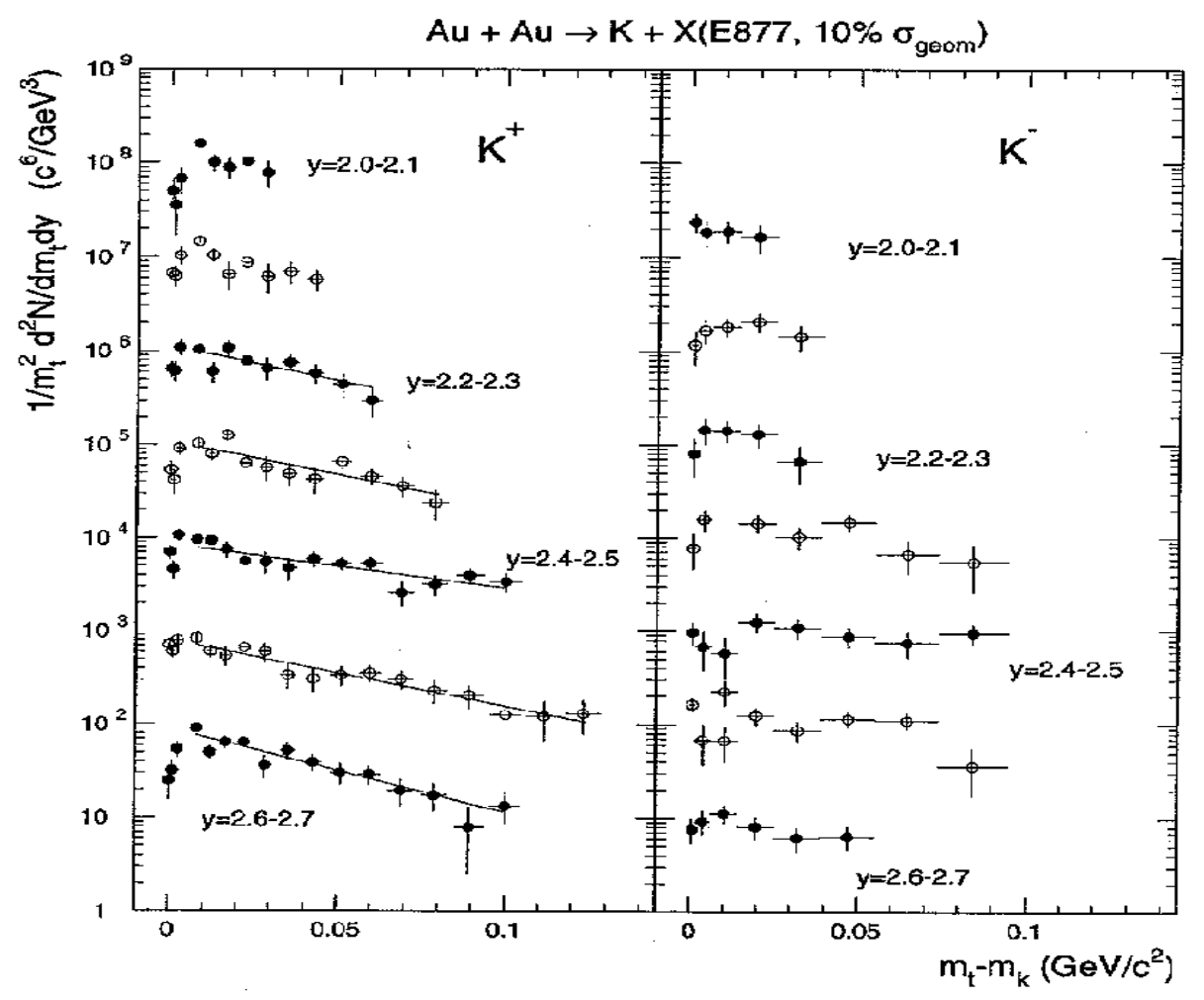

Figure 4.22: Transverse mass spectra for $\mathrm{K}^{+}$(left) and $\mathrm{K}^{-}$(right) for central $\mathrm{Au}+\mathrm{Au}$ collisions at $10 \mathrm{AGeV}$ beam energy. The data are presented in rapidity bins of 0.1 units widths successively

[174. The $\mathrm{K}^{+} / \pi^{+}$ratio (left part) increases from about 0.105 in peripheral $\mathrm{Au}+\mathrm{Au}$ collisions to about 0.16 in central collisions. It is interesting to note that the $\mathrm{K}^{+} / \mathrm{K}^{-}$stays approximately constant as a function of centrality (right part of Fig. 4.23). One would expect that due to absorption the relative $\mathrm{K}^{-}$yield decreases with increasing centrality as it was found for the antiprotons 24$]$.

The corresponding $\mathrm{K}^{+} / \pi^{+}$ratio in nucleon-nucleon collisions is 0.05 for $4 \pi$ multiplicities (which is only slightly $(10 \%)$ lower than the midrapidity value). Microscopic models interpret the three times higher $\mathrm{K}^{+} / \pi^{+}$ratio in central $\mathrm{A}+\mathrm{A}$ collisions as the result of interactions of the produced pions with the fast incoming nucleons 175, 176. "Strangeness enhancement" then means that strange mesons can populate the larger phase space which is opened by multiple hadron-hadron collisions in heavy systems. Remember that the different centrality dependence of the $\mathrm{K}^{+}$and the $\pi^{+}$multiplicity measured in $\mathrm{Au}+\mathrm{Au}$ reactions at SIS energies (see Fig 4.3) was also explained by secondary meson-baryon collisions.

At the highest energies presently available for heavy ion beams (200 AGeV) the data on symmetric systems comprise $\mathrm{S}+\mathrm{S}[34,35]$ and $\mathrm{Pb}+\mathrm{Pb}$ collisions [42]. As an example Fig. 4.24 and Fig. 4.25 show preliminary transverse mass spectra of kaons and Lambdas from $\mathrm{Pb}+\mathrm{Pb}$ collisions at $158 \mathrm{AGeV}$ measured by the NA49 Collaboration. All spectra can be described by a single exponential function in $\mathrm{m}_{T}$. The slope factors are listed in Table 4.3. The large values of about $200 \mathrm{MeV}$ and larger (see $\Lambda$ and $\bar{\Lambda}$ for $\mathrm{Pb}+\mathrm{Pb}$ ) suggest that the spectral slopes are affected by a transverse or radial flow.

Both at midrapidity and in $4 \pi$, strange particle yields at SPS energies are enhanced by a factor of about two when normalized to the pions and compared to nucleon-nucleon collisions 59. In terms of K-mesons this finding is summarized in Fig. 4.26 in which the $\mathrm{K} / \pi$ ratio is given as a function of the number of participating nucleons for $\mathrm{p}+\mathrm{p}, \mathrm{p}+\mathrm{A}, \mathrm{S}+\mathrm{A}$ and $\mathrm{Pb}+\mathrm{Pb}$ collisions [33]. However, as already mentioned above, this kind of strangeness enhancement has been observed also at SIS and AGS energies where it was explained by multiple interaction of participants. The kaon to pion ratio is an ambiguous measure of "strangeness" enhancement as one compares particles with different masses which populate phase space differently. The phase space available in $\mathrm{A}+\mathrm{A}$ collisions is enhanced as compared to the one available in $\mathrm{p}+\mathrm{p}$ collisions due to multiple nucleon-nucleon interactions. This "new" phase space is preferentially 

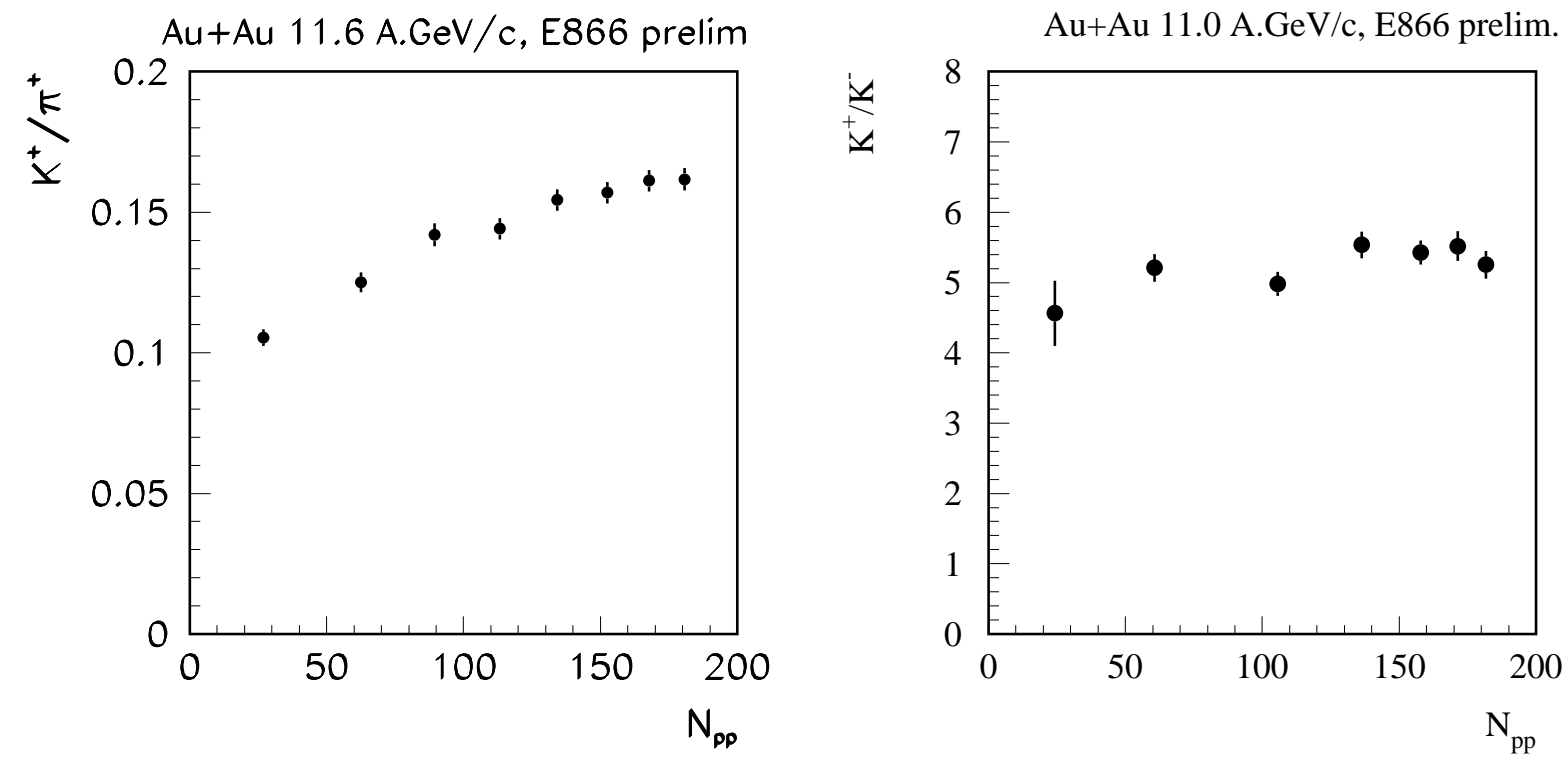

Figure 4.23: Ratio of $\mathrm{K}^{+} / \pi^{+}$(left) and $\mathrm{K}^{+} / \mathrm{K}^{-}$(right) fiducial yields as a function of the number of projectile participants for $\mathrm{Au}+\mathrm{Au}$ collisions at $10.7 \mathrm{AGeV}$ beam energy (preliminary, taken from [174]).

$\mathrm{Pb}+\mathrm{Pb}, \mathrm{NA} 49 \mathrm{~K}^{+}, \mathrm{K}^{-}, \mathrm{K}_{\mathrm{s}}^{0}$ Preliminary
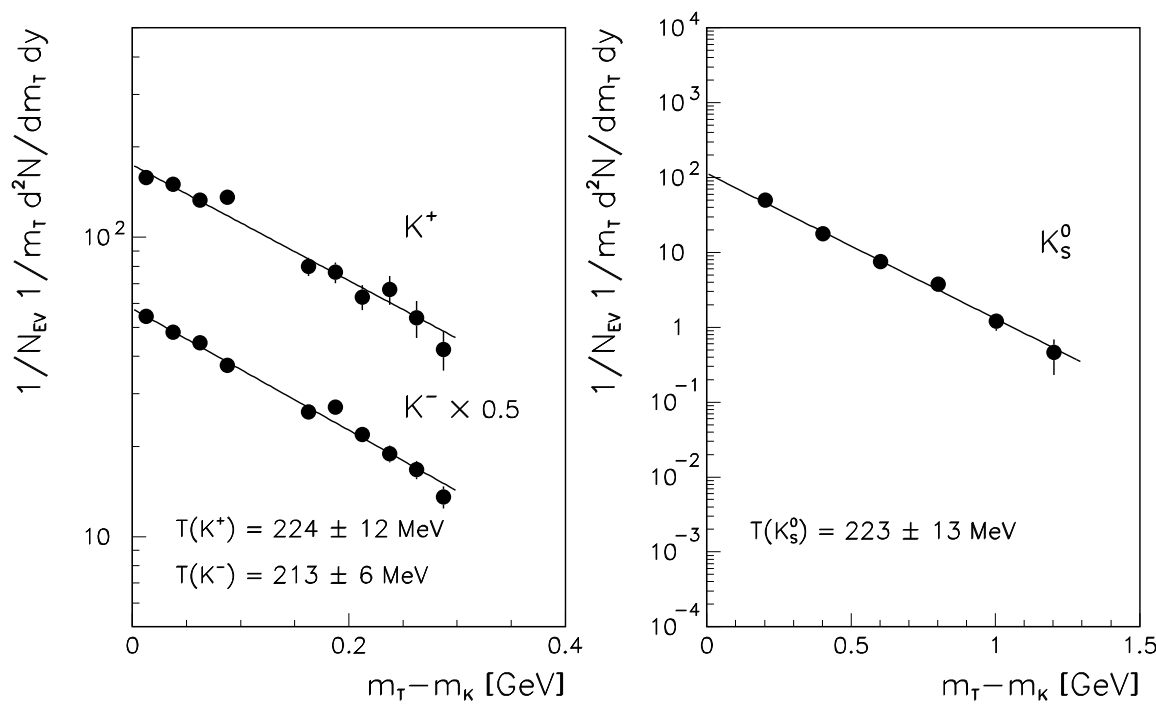

Figure 4.24: The transverse mass spectra of $\mathrm{K}^{+}$and $\mathrm{K}^{-}$mesons $(2.5<\mathrm{y}<3.3)$ and of $\mathrm{K}_{s}^{0}(2.0<\mathrm{y}<2.7)$ per unit of rapidity for $\mathrm{Pb}+\mathrm{Pb}$ collisions at $158 \mathrm{AGeV}$ [33]. 
Pb+Pb, NA49 Preliminary

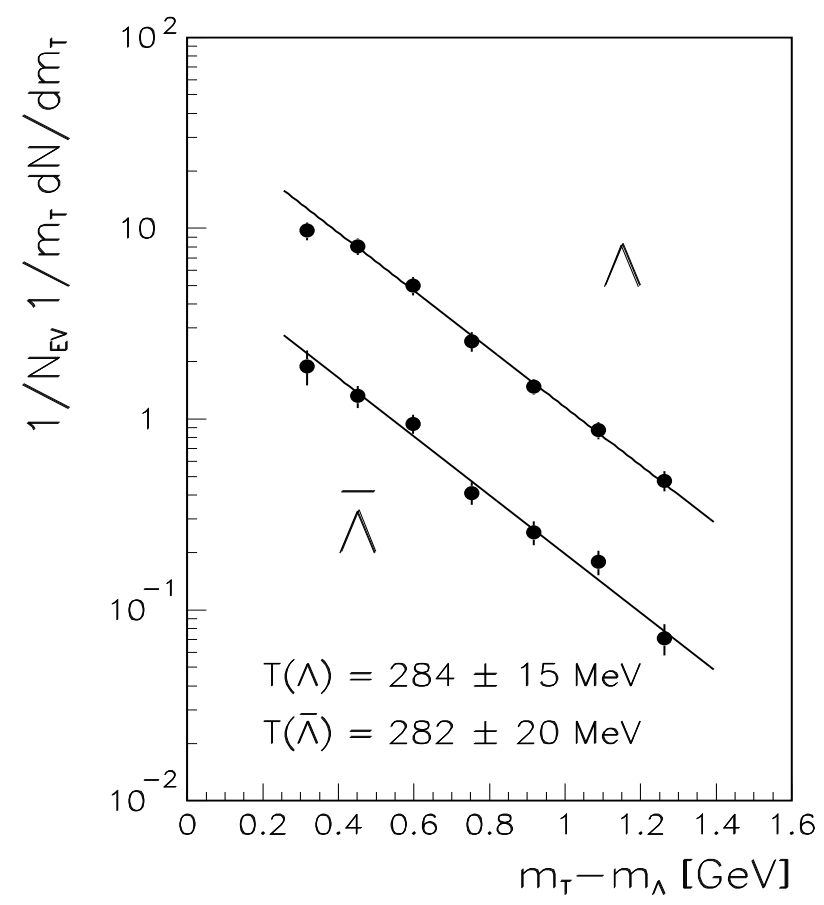

$\mathrm{Pb}+\mathrm{Pb}, \mathrm{NA} 49$ Preliminary

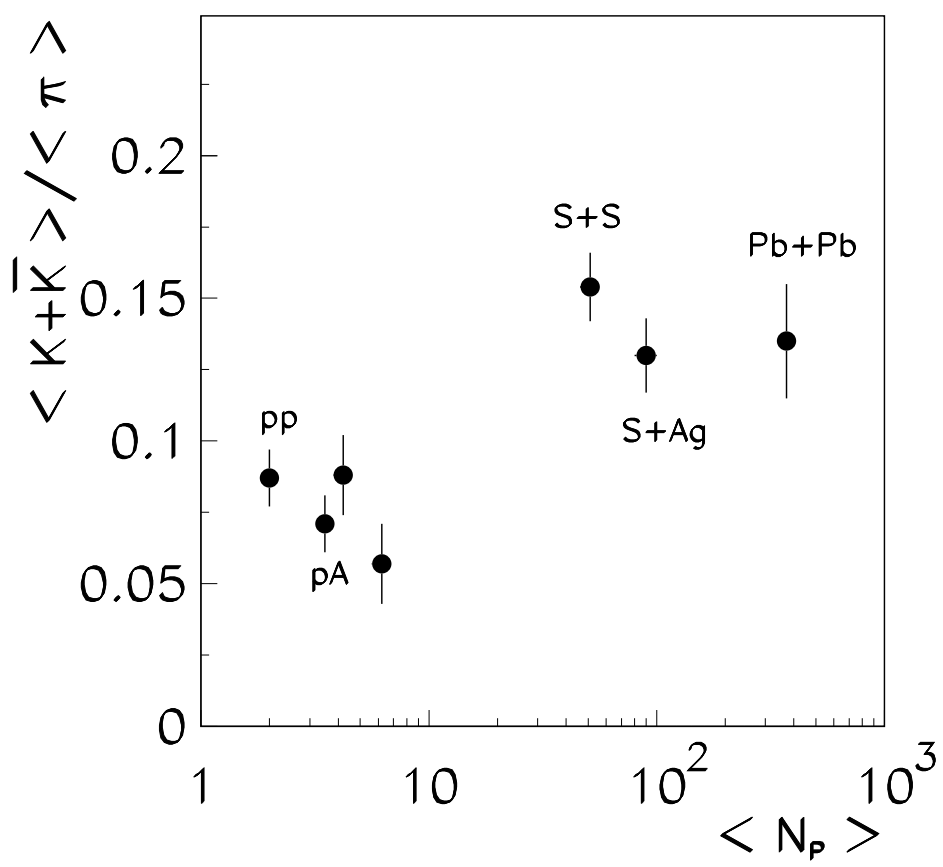

Figure 4.25: The transverse mass spectra of $\Lambda$ and $\bar{\Lambda}(2.6<\mathrm{y}<$ 3.8) for $\mathrm{Pb}+\mathrm{Pb}$ collisions at 158 $\mathrm{AGeV}$. The spectra are divided by the width of the rapidity interval [33].
Figure 4.26: The $\mathrm{K} / \pi$ ratio, defined as the sum of the multiplicities of all kaons $\left(\mathrm{K}^{+}, \mathrm{K}^{-}, 2 \mathrm{~K}_{s}^{0}\right)$ divided by all pions $\left(\pi^{+}, \pi^{-}, \pi^{0}\right)$, as a function of the number of participant nucleons for $\mathrm{Pb}+\mathrm{Pb}$ collisions at $158 \mathrm{AGeV}$ 33. 
Table 4.3: Inverse slope parameters $\mathrm{T}$ fitted to the the transverse mass distributions according to $\mathrm{d} \sigma / \mathrm{dm}_{T}$ $\propto \mathrm{m}_{T} \exp \left(-\mathrm{m}_{T} / \mathrm{T}\right)$ The data are taken from [34, 35, 42].

\begin{tabular}{|c|c|c|c|c|c|}
\hline reaction & $\Lambda$ & $\bar{\Lambda}$ & $\mathrm{K}_{S}^{0}$ & $\mathrm{~K}^{+}$ & $\mathrm{K}^{-}$ \\
\hline $\mathrm{p}+{ }^{32} \mathrm{~S}$ & $182 \pm 17$ & $132 \pm 18$ & $205 \pm 16$ & $227 \pm 52$ & $286 \pm 106$ \\
\hline${ }^{32} \mathrm{~S}+\mathrm{S}$ & $204 \pm 17$ & $180 \pm 24$ & $210 \pm 16$ & $227 \pm 15$ & $270 \pm 37$ \\
\hline${ }^{32} \mathrm{~S}+\mathrm{Ag}$ & $234 \pm 17$ & $221 \pm 24$ & $231 \pm 17$ & $254 \pm 31$ & $181 \pm 28$ \\
\hline${ }^{32} \mathrm{~S}+\mathrm{Au}$ & $240 \pm 18$ & $223 \pm 22$ & $227 \pm 18$ & - & - \\
\hline${ }^{208} \mathrm{~Pb}+\mathrm{Pb}$ & $293 \pm 10$ & $288 \pm 15$ & $223 \pm 13$ & $224 \pm 12$ & $213 \pm 6$ \\
\hline
\end{tabular}

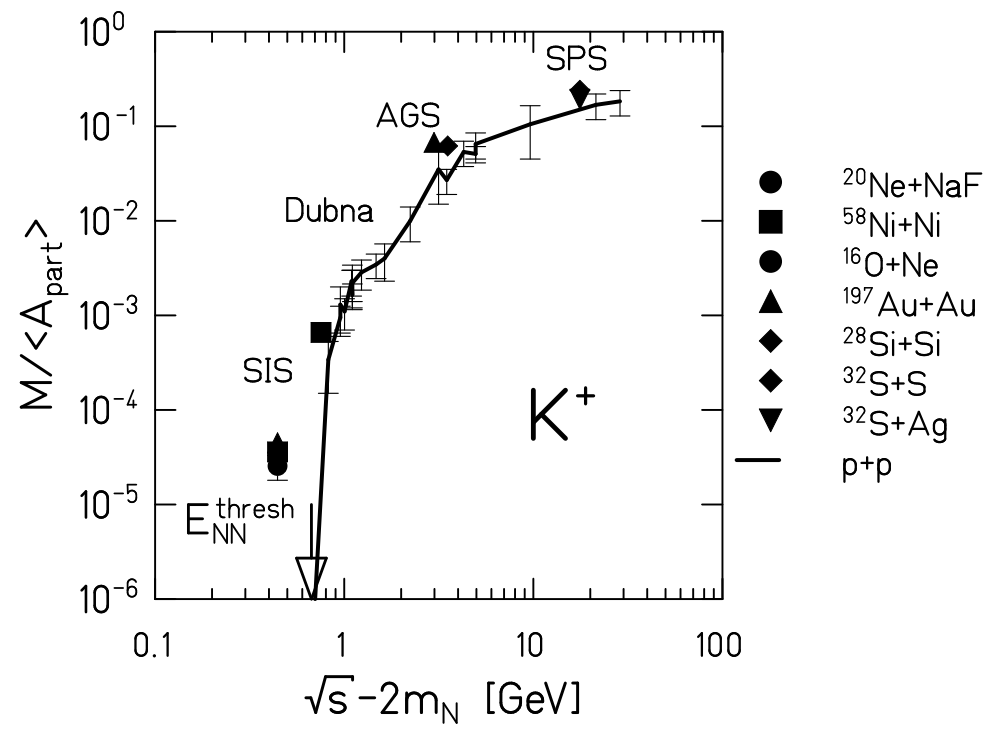

Figure 4.27: $\mathrm{K}^{+}$multiplicity per average number of participants for $\mathrm{A}+\mathrm{A}$ (full symbols) and $\mathrm{p}+\mathrm{p}$ collisions (solid line with error bars) as a function of the energy available in the nucleon-nucleon system. The data are taken from [59, 110, 111].

populated by particles with high energies or large masses. For example, at SIS energies the yield of highenergy pions increases (like the $\mathrm{K}^{+}$yield) much faster than the low-energy pion yield when increasing the mass of the collision system [82]. Therefore, one should consider for example the kaon to eta ratio or the kaon to high-energy pion ratio as an experimental indicator for strangeness enhancement. In these ratios, simple phase space effects largely cancel out. A possible candidate in this respect is also the $\bar{\Lambda} / \bar{p}$ ratio which will be discussed in the next chapter. On the other hand, model calculations take kinematical effects into account but are not able to reproduce the strangeness data for the SPS energies [177, 178].

The $\phi$ meson is a special strange particle as it consists of a strange and antistrange quark. First results from $\mathrm{S}+\mathrm{W}$ collisions confirmed the strangeness enhancement also in this observable 179 . Recently preliminary data on $\phi$ s produced in central $\mathrm{Pb}+\mathrm{Pb}$ collisions have been presented [180]; both the shape of the transverse momentum distribution, which indicates a rather large slope factor of $\approx 350 \mathrm{MeV}$ and the large $4 \pi$ yield fit into the systematics of a correlation between slope factor and particle mass and the relative strange particle enhancement.

In the following we discuss the excitation function of kaon and antikaon production in nuclear collisions from SIS to SPS energies. Fig. 4.27 and Fig. 4.28 show the measured $\mathrm{K}^{+}$and $\mathrm{K}^{-}$multiplicities per participating nucleons as a function of the beam energy which is expressed as the sum of the kinetic 


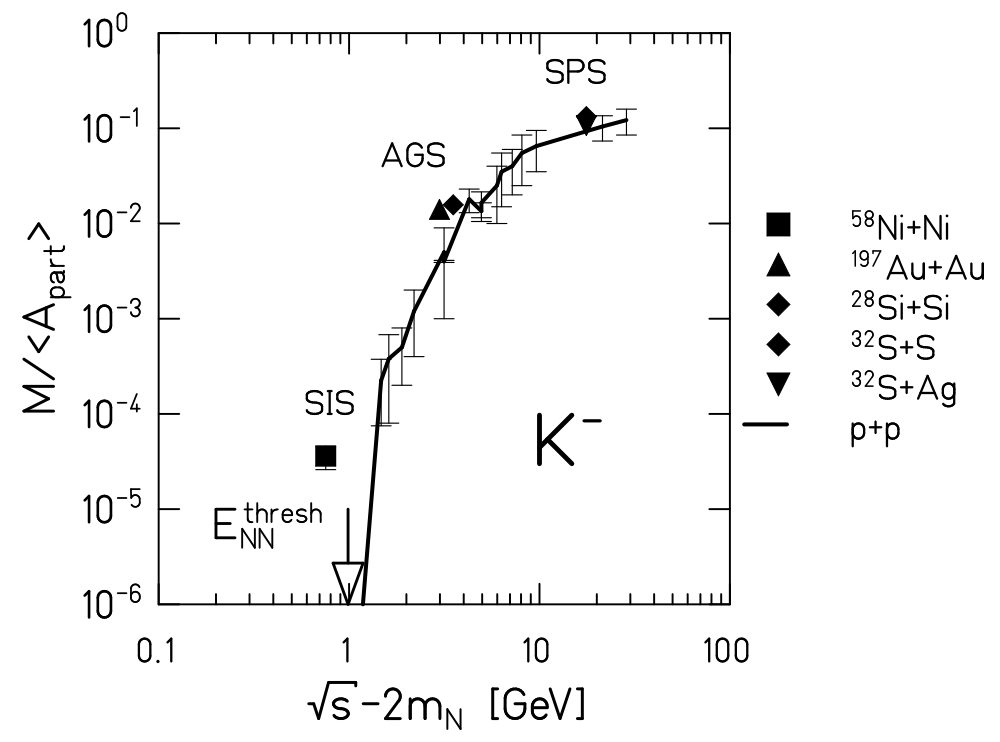

Figure 4.28: $\mathrm{K}^{-}$multiplicity per average number of participants for $\mathrm{A}+\mathrm{A}$ (full symbols) and $\mathrm{p}+\mathrm{p}$ collisions (solid line with error bars) as a function of the energy available in the nucleon-nucleon system. The data are taken from [59, 111].

energies of two nucleons in their c.m. system. This is the energy available for the production of new particles. The data are taken from [59, 110, 111. The kaon yields per participating nucleon are larger in $\mathrm{A}+\mathrm{A}$ collisions (full symbols) than in $\mathrm{p}+\mathrm{p}$ collisons (solid line with error bars) for all beam energies. The difference between $\mathrm{A}+\mathrm{A}$ and $\mathrm{p}+\mathrm{p}$ data can be interpreted as an increase of the effective beam energy in $\mathrm{A}+\mathrm{A}$ collisions. This energy shift scales approximately with the c.m. kinetic energy of the colliding nucleons $\mathrm{E}_{k i n}^{N N}\left(=\sqrt{s}-2 \mathrm{~m}_{N}\right)$ :

$$
\frac{M_{K}^{p p}}{A_{\text {part }}}\left(E_{\text {kin }}^{N N}\right)=\frac{M_{K}^{A A}}{A_{\text {part }}}\left(\alpha \times E_{\text {kin }}^{N N}\right)
$$

with $\alpha=1.3-2$. This scaling behaviour suggests that in $\mathrm{A}+\mathrm{A}$ collisions the average available energy in a nucleon pair (which produces a K meson) is $1.3-2$ times larger than in a free $\mathrm{p}+\mathrm{p}$ collision independent of the energy regime.

In section 2.3.1 we have shown that in $\mathrm{p}+\mathrm{p}$ collisions at SPS energies the protons lose on average nearly half of their kinetic energy by particle production. In A+A collisions the situation is more difficult to interpret. The rapidity distributions (normalized to beam rapidity) of the final state protons from central A+A collisions look similar at all beam energies suggesting a similar stopping power. In contrast, the inelasticity as defined in section 2.3.1 (mainly by pion production) is increasing by a factor of about 10 from SIS to SPS energies in central collisions of heavy nuclei. We attribute this discrepancy to the reabsorption of pionic (i.e. produced) energy by the nucleons. This effect is most pronounced at low beam energies. $\mathrm{K}^{+}$mesons cannot be reabsorbed and thus represent an ideal probe for the energy available for particle production in $\mathrm{p}+\mathrm{p}$ and in $\mathrm{A}+\mathrm{A}$ collisions. Our finding that $\alpha$ is between 1.3 and 2 (independent of beam energy) suggests that the nuclear stopping power in $\mathrm{Au}+\mathrm{Au}$ collisions is up to a factor of 2 higher than in $\mathrm{p}+\mathrm{p}$ collisions at all energies.

It is surprising that $\alpha$ is the same for $\mathrm{K}^{+}$and $\mathrm{K}^{-}$mesons. This means that $\mathrm{K}^{-}$absorption is not visible in spite of the large cross section for strangeness exchange reactions $\mathrm{K}^{-} \mathrm{N} \rightarrow \pi \Lambda$ (or $\Sigma$ ) of 40-70 $\mathrm{mb}$ depending on relative momentum. Unfortunately, the large uncertainties on the $\mathrm{K}^{+}$and $\mathrm{K}^{-}$yields in the $\mathrm{p}+\mathrm{p}$ data preclude any far reaching conclusion. This deficiency of the $\mathrm{p}+\mathrm{p}$ data asks for new experiments with the aim to provide a solid basis for quantitative comparisons with $\mathrm{A}+\mathrm{A}$ data. 


\section{Chapter 5}

\section{Production of etas, antibaryons and multistrange hyperons}

\section{$5.1 \quad \eta$ mesons}

The meson production experiments at the BEVALAC concentrated on charged mesons. At SIS, the TAPS collaboration started a program to measure neutral mesons with a photon detector 181. The main goal was to study $\pi^{0}$ and $\eta$-meson production at beam energies near the threshold which is $\mathrm{E}_{N N}^{t h}=1254 \mathrm{MeV}$ for free nucleon-nucleon collisions. Eta mesons originate almost exclusively from the decay of the $\mathrm{N}^{*}(1535)$ resonance and thus are sensitive to the abundance of this resonance.

Fig. 5.1 shows the first results on the $\eta$ transverse momentum distribution measured at midrapidity for $\mathrm{Ar}+\mathrm{Ca}$ at 1.0 and $1.5 \mathrm{AGeV}, \mathrm{Kr}+\mathrm{Zr}$ and $\mathrm{Au}+\mathrm{Au}$ at $1.0 \mathrm{AGeV}$ 70. The simultaneous measurement of $\eta$ and $\pi^{0}$ mesons allow to determine the $\eta / \pi^{0}$ ratio with reduced systematic errors. After extrapolation to full phase space, the $\eta / \pi^{0}$ ratios are found to be $1.3 \pm 0.8 \%$ at $1.0 \mathrm{AGeV}$ and $2.2 \pm 0.4 \%$ at $1.5 \mathrm{AGeV}$. From the $\eta / \pi^{0}$ ratio the relative abundance of $\Delta(1232)$ and $N^{*}(1535)$ at their respective freeze-out has been estimated. Assuming $\Delta \rightarrow \pi \mathrm{N}$ to be the dominant pion production channel and taking into account the appropriate isospin factors together with a branching ratio of $40 \%$ for the $\mathrm{N}^{*}(1535)$ decay into the $\eta$ channel, the TAPS collaboration found a $\mathrm{N}^{*}(1535) / \Delta(1232)$ ratio of $(1.1 \pm 0.6) \%$ and $(1.8 \pm 0.3) \%$ at 1.0 and $1.5 \mathrm{AGeV}$, respectively [70].

Recently, $\eta$ mesons were measured in the light system $\mathrm{C}+\mathrm{C}$ at different bombarding energies. Fig. 5.2 shows the production cross sections for $\pi^{0}$ and $\eta$ mesons in a Boltzmann representation as a function of the transverse mass [53]. The $\eta$ and $\pi^{0}$ spectra taken at $2.0 \mathrm{AGeV}$ fall on top of each other if plotted as a function of the transverse mass $\mathrm{m}_{T}$. Such a $\mathrm{m}_{T}$ scaling is expected (but no proof) for a thermally and hadrochemically equilibrated source. In any case it is worthwhile to note that the pions perfectly follow a Boltzmann distribution although they are emitted from a source which on average consists of 6 (participant) nucleons only.

Fig. 5.3 presents the neutral meson production probability per participant nucleon a as a function of the bombarding energy for different collision systems 53 . The beam energy is normalized to the respective threshold energy for free NN collisions. The eta and pion data follow a common trend which means that their production probability just depends on the available energy. Charged pions also fit into this picture.

The abundances of resonances like $\Delta(1232)$ and $\mathrm{N}^{*}(1535)$ as derived from the pion and eta yields are in agreement with the assumption of thermal and hadrochemical equilibrium. This is demonstrated in Fig. 5.4 which compares the meson yields (full symbols) to the resonance population at chemical freeze-out (shaded areas) for different available energies. The resonance population was calculated for a given temperature $\mathrm{T}$ and baryochemical potential $\mu_{B}$ [13]. The parameter set $\left(\mathrm{T}, \mu_{B}\right)$ was determined from particle production ratios $\pi / \mathrm{A}_{\text {part }}, \eta / \pi^{0}$ and from production rates of $\pi, \Delta, \mathrm{p}, \mathrm{d}$ for different collisions systems and bombarding energies 13] (see chapter 2). Up to $15 \%$ of the nucleons are found to be excited to resonance states at freeze-out for the largest value of available energy (which corresponds to a beam energy of $2 \mathrm{AGeV}$ ). 


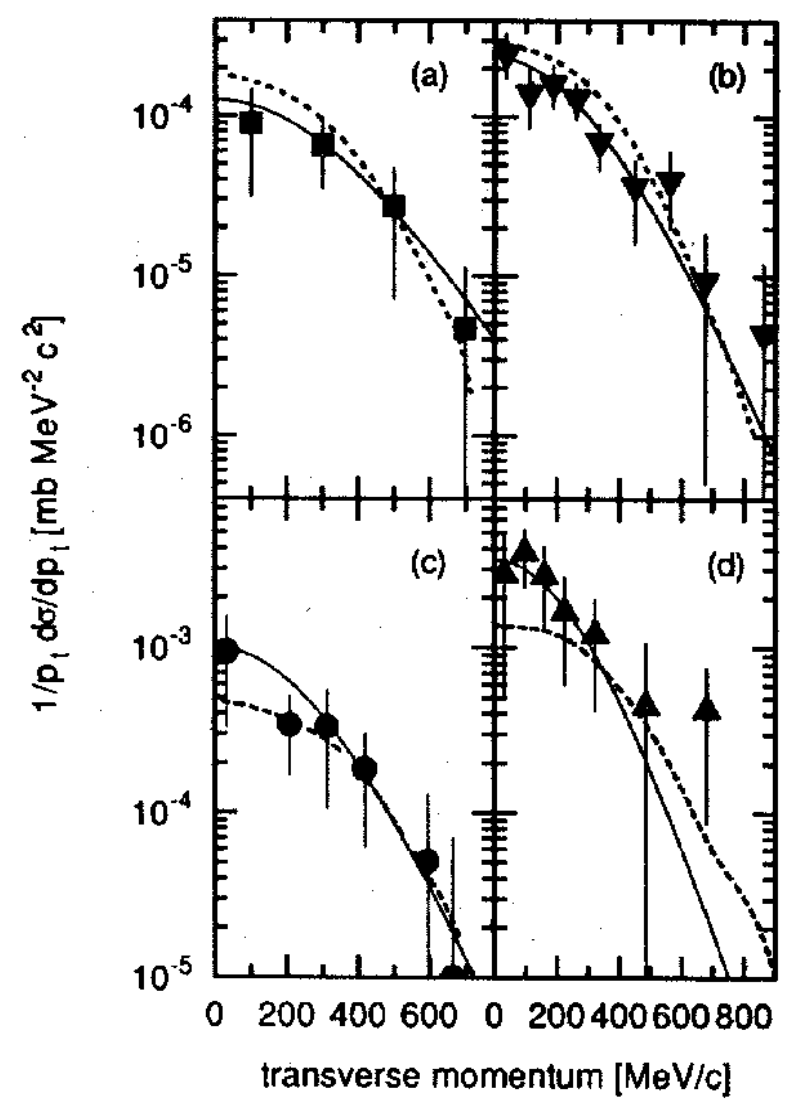

Figure 5.1: Transverse momentum distributions at midrapidity for the systems Ar+Ca at 1.0 (a) and 1.5 $\mathrm{AGeV}(\mathrm{b}), \mathrm{Kr}+\mathrm{Zr}(\mathrm{c})$ and $\mathrm{Au}+\mathrm{Au}(\mathrm{d})$ at $1.0 \mathrm{AGeV}[70]$. The solid lines represent fits with a thermal, isotropic source at midrapidity while the dashed curves show results of BUU calculations 182.

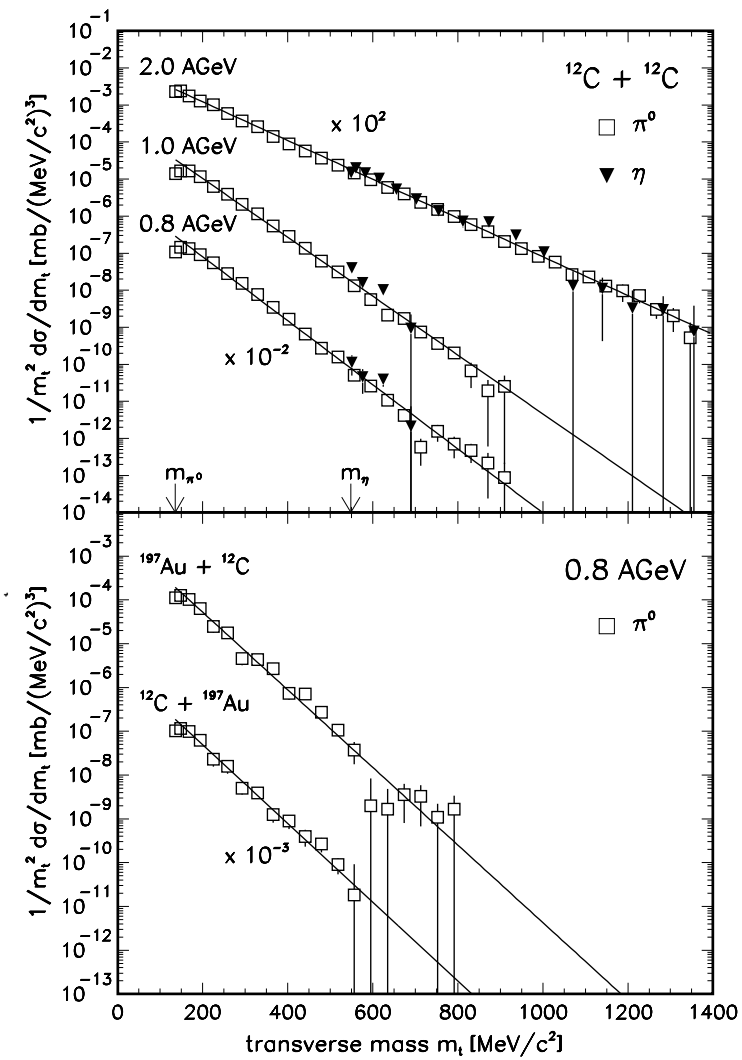

Figure 5.2: Transverse mass spectra of $\pi^{0}$ and $\eta$ mesons measured in the system $\mathrm{C}+\mathrm{C}$ at beam energies of $0.8,1.0$ and $2.0 \mathrm{AGeV}$ within intervals of rapidity of $0.42-0.74,0.42-0.74$ and $0.8-1.08$, respectively (upper frame). Transverse mass spectra of $\pi^{0}$ are measured in $\mathrm{C}+\mathrm{Au}$ and $\mathrm{Au}+\mathrm{C}$ at $0.8 \mathrm{AGeV}$ at rapidities of $0.42-0.74$ (lower frame). The lines represent fits to the pion data according to $d \sigma / d m_{T} \propto m_{T}^{2} \exp \left(-m_{T} / T\right)$ which corresponds to the assumption of a thermal and isotropic meson source [53]. 


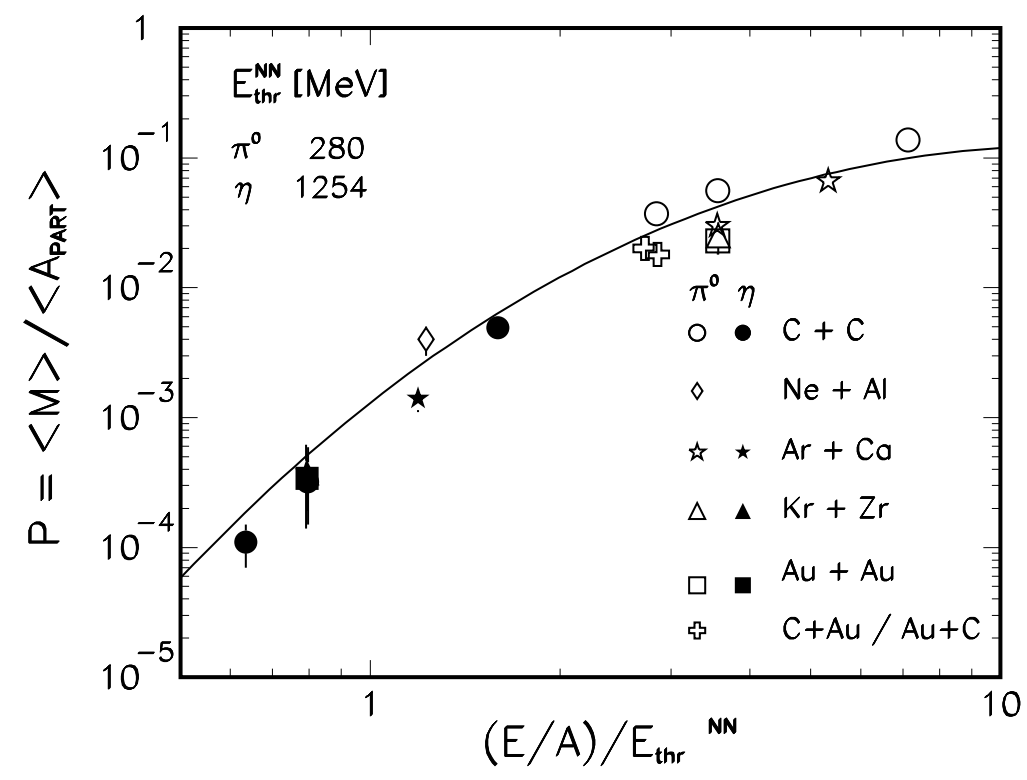

Figure 5.3: Average $\pi^{0}$ and $\eta$ multiplicities per average number of paticipants in $\mathrm{A}+\mathrm{A}$ collisions as a function of the bombarding energy, corrected for energy loss in the target and normalized to the production thresholds. The picture is taken from [53].

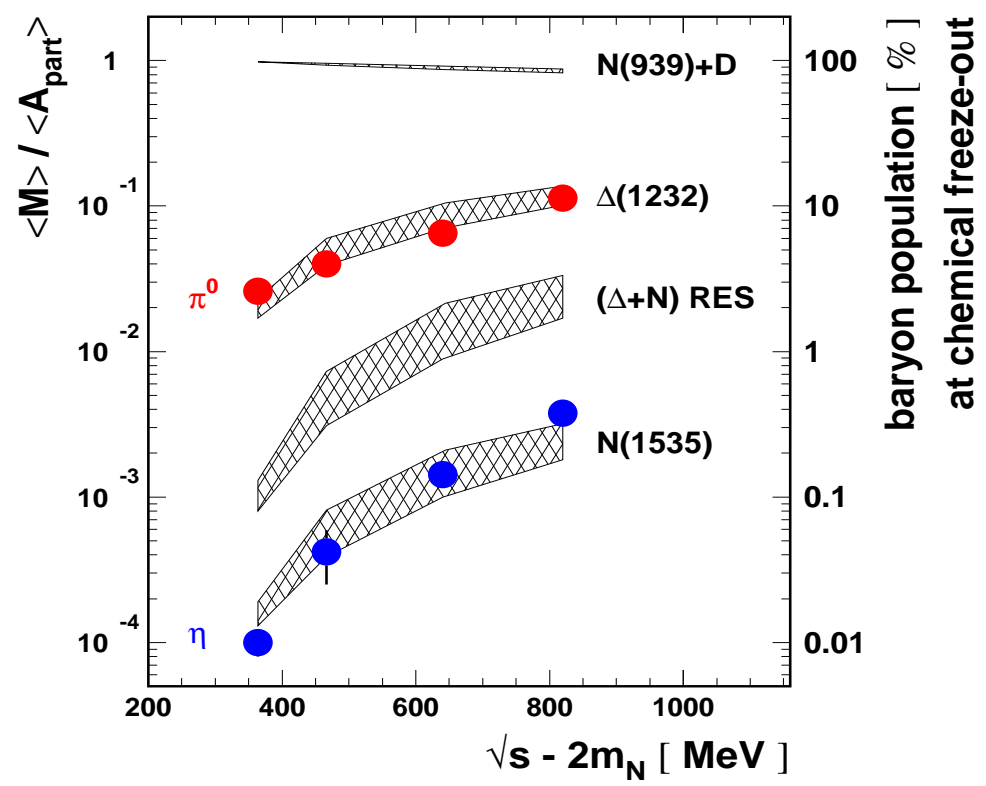

Figure 5.4: Inclusive $\pi^{0}$ and $\eta$ multiplicities per average number of participants (left scale) as a function of the energy available in the nucleonnucleon system. The hatched areas show the relative populations of nucleons and deuterons, of the $\Delta(1332)$ resonance, of the $\mathrm{N}(1535)$ resonance and of the sum of all remaining $\Delta$ and $\mathrm{N}$ resonances (right scale), as obtained from a hadrongas model 13]. 


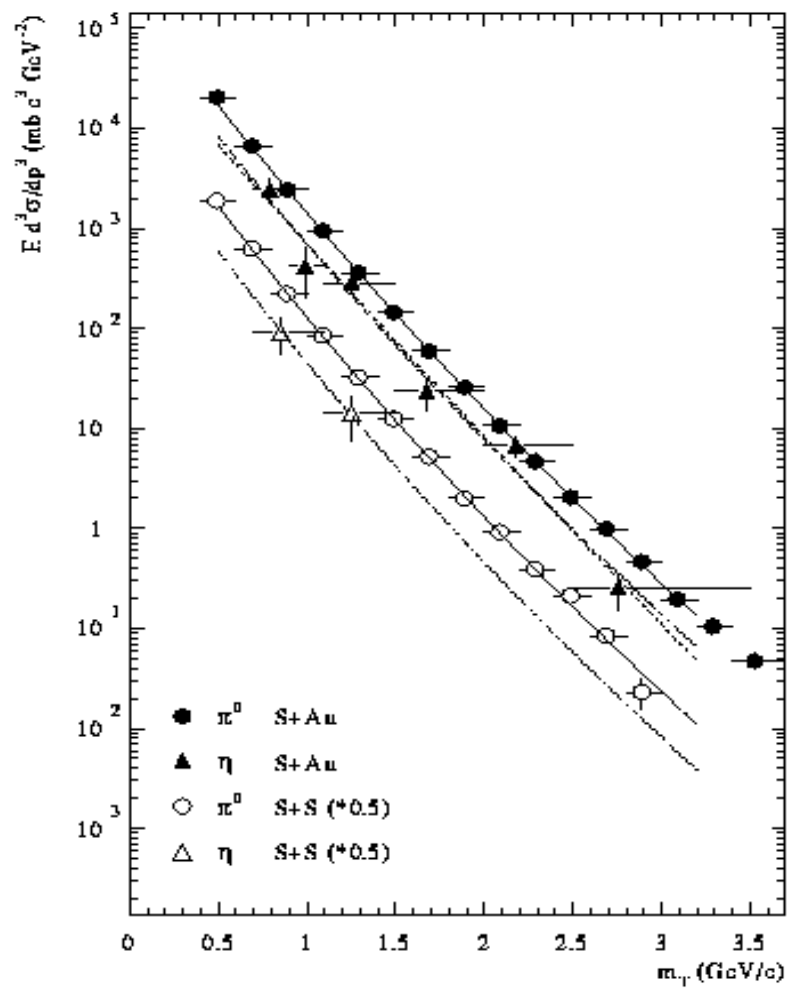

Figure 5.5: Invariant cross sections of $\pi^{0}$ and $\eta$ mesons averaged over the rapidity interval of $2.1 \leq y \leq 2.9$ as a function of transverse mass for $200 \mathrm{AGeV} \mathrm{S}+\mathrm{Au}$ and $\mathrm{S}+\mathrm{S}$ minimum bias data. The $\mathrm{S}+\mathrm{S}$ data have been scaled by a factor of 0.5 for better presentation 183 .

Eta production was also measured at SPS energies by the WA80 Collaboration. In $\mathrm{S}+\mathrm{Au}$ and $\mathrm{S}+\mathrm{S}$ collisions at $200 \mathrm{AGeV} / \mathrm{c}$ the $\eta / \pi^{0}$ cross section ratio was found to be $0.12 \pm 0.04$ 183. Fig. 5.5 presents the invariant cross sections of $\pi^{0}$ and $\eta$ mesons averaged over the rapidity interval of $2.1 \leq \mathrm{y} \leq 2.9$ as a function of transverse mass. Etas and pions roughly follow $\mathrm{m}_{T}$ scaling with an inverse slope parameter of $\mathrm{T}=226 \pm 9 \mathrm{MeV}$ with a slightly smaller value for the pions. This deviation suggests a radial expansion velocity at freeze-out of approximatelly $\beta=0.3-0.5$.

\subsection{Antibaryons}

The production of a proton-antiproton pair in a proton-proton collision requires a beam energy of $5.6 \mathrm{GeV}$. Nonetheless, antiprotons were observed at the BEVALAC/LBL in 1.65 and $2 \mathrm{AGeV} \mathrm{Si+Si} \mathrm{and} 2 \mathrm{AGeV}$ $\mathrm{Ne}+\mathrm{NaF}$ collisions [54], at the SIS/GSI with $\mathrm{Ne}$ and $\mathrm{Ni}$ beams of $1.66-1.93 \mathrm{AGeV}$ on various targets 555 and in $\mathrm{C}+\mathrm{C}$ collisions at $3.65 \mathrm{AGeV}$ at the JINR in Dubna [184. In order to suppress sufficiently the pions and antikaons - which are typically 7 and 3 orders of magnitude more abundant than antiprotons the measurements at LBL and GSI have been performed at 0 degree with a small-acceptance beam-line spectrometer.

Although antiproton production is extremely subthreshold at BEVALAC/SIS energies, it seems to follow an universal trend. It was pointed out that the antiprotons fit into a scaling pattern together with the pions and kaons when plotting the invariant cross sections over the energy needed to produce the particles [54, 136. This energy is the sum of the particle c.m. kinetic energy and the c.m. threshold energy: $\mathrm{E}^{*}$ $=\mathrm{E}_{\text {kin }}^{c . m}+\mathrm{E}_{\text {thresh }}$. The threshold energy $\mathrm{E}_{\text {thresh }}$ is the minimum energy required to produce the particle in a $\mathrm{NN}$ collision and is equal to $\mathrm{m}_{\pi}$ for pion production, $\mathrm{m}_{K}+\mathrm{m}_{\Lambda^{-}} \mathrm{m}_{N}$ for $\mathrm{K}^{+}$production, $2 \mathrm{~m}_{K}$ for $\mathrm{K}^{-}$ production and $2 \mathrm{~m}_{p}$ for antiproton production. The invariant particle production cross sections fall on an exponential $\mathrm{E} d^{3} \sigma / d p^{3} \propto \exp \left(-\mathrm{E}^{*} / \mathrm{T}\right)$ with $\mathrm{T}$ the inverse slope parameter depending on beam energy and on the size of the colliding system. This scaling was found for $\mathrm{Si}+\mathrm{Si}(\mathrm{Al})$ collisions both at BEVALAC 

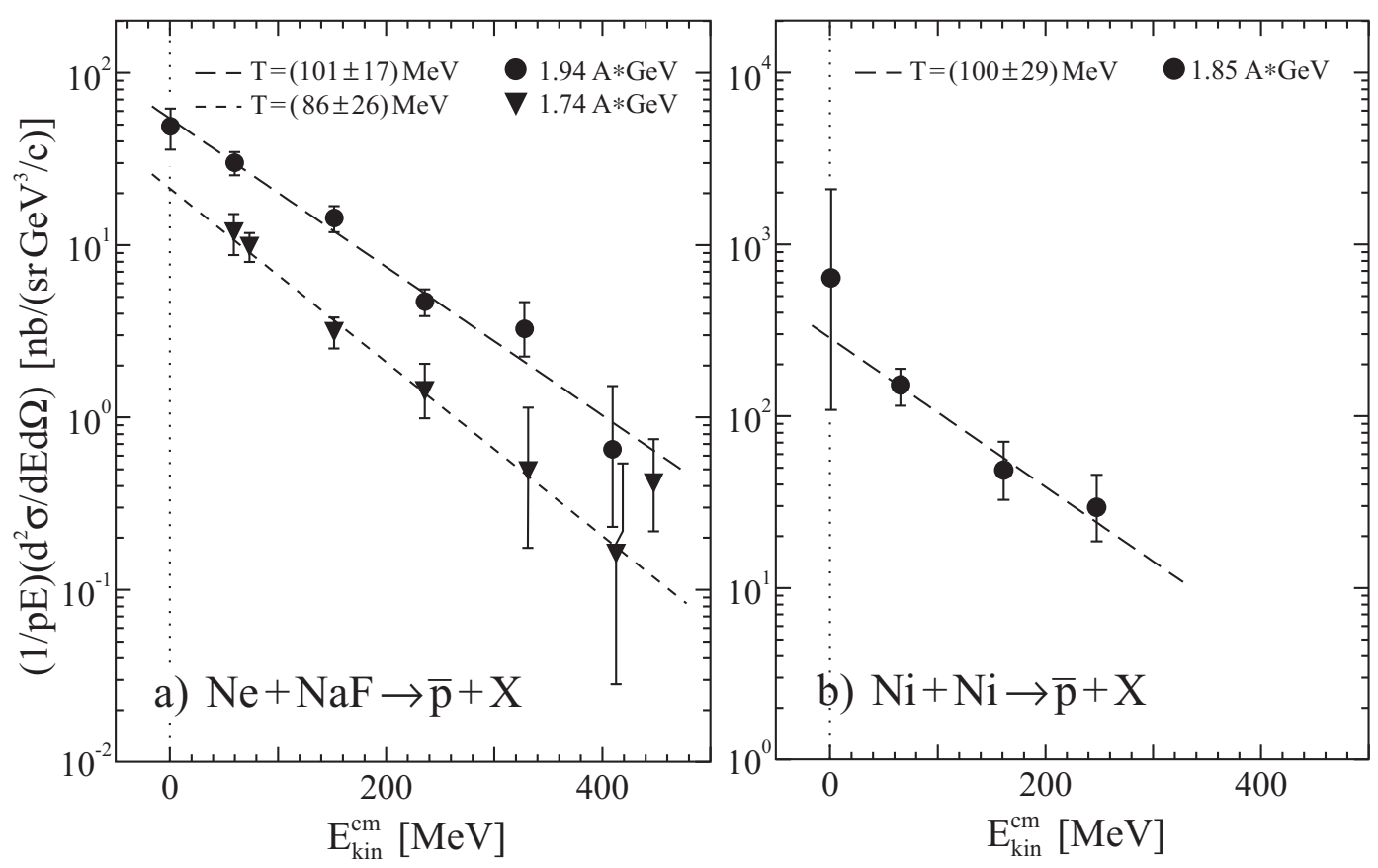

Figure 5.6: Invariant $\bar{p}$ production cross sections as a function of the c.m. kinetic energy for the reactions $\mathrm{Ne}+\mathrm{NaF}$ at 1.74 and $1.94 \mathrm{AGeV}$ (left) and $\mathrm{Ni}+\mathrm{Ni}$ at $1.85 \mathrm{AGeV}$ (right) 138. The dashed lines are exponential fits to the data with inverse slope parameters $\mathrm{T}$ as indicated.

and at AGS energies 136]. Surprisingly enough, the universal scaling holds also for antiprotons which are expected to be strongly absorbed. The FRS/GSI data confirmed the scaling law for $\mathrm{Ne}+\mathrm{NaF}$ collisions but found deviations for the heavier $\mathrm{Ni}+\mathrm{Ni}$ system $[138]$.

The phenomenon of deep subthreshold particle production opens the possibility to study (i) the mechanisms of energy accumulation via multiple hadron-hadron collisions or short-range correlations etc. and (ii) in medium modifications of cross sections, effective masses, thresholds etc. The Boltzmann distributions and the scaling pattern of the produced particles suggest a thermal origin. Therefore, thermal fireball models have been proposed to explain the measured particle yields. As an example we mention the ansatz of Ref. 185]. The authors assume that only nucleons and $\Delta$ resonances are in thermal and chemical equilibrium. Kaons, antikaons and antiprotons are not equilibrated but created (and possibly absorbed) in thermal baryon-baryon collisions. The particle abundances are calculated by rate equations. The model uses free production cross sections and allows for a cooling of the fireball. The pions are not considered to be in equilibrium but are created via decays of $\Delta$ resonances after freeze-out. Therefore, $\pi \mathrm{N}$ collisions are omitted in this approach. The thermal equilibrium of baryons guarantees the population of the very high energy tails of the Boltzmann distribution. These energies are required for deep subthreshold processes. The $\mathrm{K}^{-} / p$ and $\bar{p} / \mathrm{p}$ ratios measured in $\mathrm{Si}+\mathrm{Si}$ collisions at $2 \mathrm{AGeV}$ at the BEVALAC 54 could be reproduced by this model. Antiproton production in $\mathrm{C}+\mathrm{C}$ collisions could not be explained; this was considered as an indication for a non-equilibrated system.

Fig. 5.6 shows the invariant $\bar{p}$ production cross sections as a function of the $\bar{p}$ c.m. kinetic energy measured in $\mathrm{Ne}+\mathrm{NaF}$ and $\mathrm{Ni}+\mathrm{Ni}$ collisions by the FRS group at SIS 138. The spectral slopes follow exponentials as indicated by the dashed lines. The inverse slope parameters are $101 \pm 17 \mathrm{MeV}$ and $86 \pm 26$ $\mathrm{MeV}$ for $\mathrm{Ne}+\mathrm{NaF}$ collisions (at 1.94 and $1.74 \mathrm{AGeV}$ ) and $100 \pm 29 \mathrm{MeV}$ for $\mathrm{Ni}+\mathrm{Ni}$ collisions at $1.85 \mathrm{AGeV}$. These values agree with the ones for the corresponding $\mathrm{K}^{-}$mesons and for the high-energy tail of the $\pi^{-}$mesons [138]. The data shown in Fig. 5.6 allow to extract information on the mass dependence of antiproton production and on the excitation function.

The ratio of the invariant cross sections for $\mathrm{Ni}+\mathrm{Ni} \rightarrow \bar{p}+\mathrm{X}$ and $\mathrm{Ne}+\mathrm{NaF} \rightarrow \bar{p}+\mathrm{X}$ was found to be $\sigma_{N i}^{\bar{p}} / \sigma_{N e}^{\bar{p}}$ 


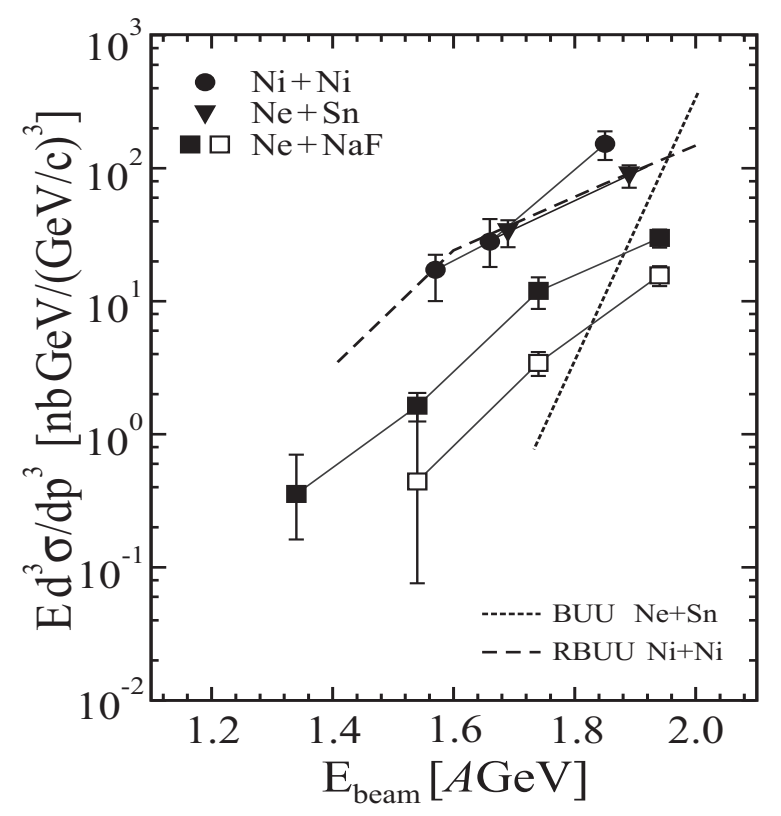

Figure 5.7: Excitation functions for $\bar{p}$ production for $\mathrm{Ni}+\mathrm{Ni}$, $\mathrm{Ne}+\mathrm{Sn}$ and $\mathrm{Ne}+\mathrm{NaF}$ collisions with respect to the beam energy 138. The $\mathrm{Ni}+\mathrm{Ni}$ data are taken at $\mathrm{p}_{c m}=0.34 \mathrm{GeV} / \mathrm{c}$, the $\mathrm{Ne}+\mathrm{NaF}$ data at $\mathrm{p}_{c m}=$ $0.34 \mathrm{GeV} / \mathrm{c}$ (full squares) and $0.56 \mathrm{GeV} / \mathrm{c}$ (open squares), the $\mathrm{Ne}+\mathrm{Sn}$ data at $\mathrm{p}_{\mathrm{cm}}=1.5 \mathrm{GeV} / \mathrm{c}$. The lines represent calculations without (dotted line, BUU) and with in-medium mass reduction of the $\bar{p}$ (dashed line, RBUU) [189.

$=9 \pm 3$ 138. When correcting for the geometrical reaction cross section one obtains the $\bar{p}$ multiplicities $\mathrm{M}^{\bar{p}}=\sigma^{\bar{p}} / \sigma_{R}$ with $\sigma_{R}=2.7 \mathrm{~b}$ for $\mathrm{Ni}+\mathrm{Ni}$ and $1.3 \mathrm{~b}$ for $\mathrm{Ne}+\mathrm{NaF}$. Hence the ratio of $\bar{p}$ multiplicities is $\mathrm{M}_{N i}^{\bar{p}} / \mathrm{M}_{N e}^{\bar{p}}=4.3 \pm 1.4$. According to a geometrical model, the average number of participants is $<\mathrm{A}_{\text {part }}>$ $=10.2$ for impact parameter integrated $\mathrm{Ne}+\mathrm{NaF}$ collisions and $\left\langle\mathrm{A}_{\text {part }}\right\rangle=29.2$ for $\mathrm{Ni}+\mathrm{Ni}$ [55]. These numbers show that the $\bar{p}$ multiplicity increases somewhat stronger than the number of participants.

However, the measured $\bar{p}$ yields (and their ratios) reflect a subtle interplay of production and absorption processes. The main problem in the interpretation of the antiproton data is the large probability for $\bar{p}$ annihilation. The antiproton annihilation cross section in $\bar{p}$ p collisions for a $\bar{p}$ momentum of $1 \mathrm{GeV} / \mathrm{c}$ is about $70 \mathrm{mb} 186$ corresponding to a mean free path of $\lambda=0.9 \mathrm{fm}$ in normal nuclear matter. As the antiproton is detected under $\Theta_{l a b}=0^{\circ}$ one can assume, that it has to propagate through a piece of nuclear matter which has the dimension of the radius of the target nucleus $\left(\mathrm{R}=1.2 \times \mathrm{A}^{1 / 3} \mathrm{fm}\right)$. According to $\exp (-$ $\mathrm{R} / \lambda$ ) only about $3 \%$ of the primordial $\bar{p}$ yield reaches the detector for $\mathrm{Ne}+\mathrm{NaF}$ collisions and about $0.6 \%$ for $\mathrm{Ni}=\mathrm{Ni}$ collisions. These numbers agree reasonably well with the result of a RBUU calculation which finds a $\bar{p}$ survival rate of about $1 \%$ for $\mathrm{Si}+\mathrm{Si}$ collisions at a $\bar{p}$ momentum of $1 \mathrm{GeV} / \mathrm{c}$ [187]. When taking the estimated losses into account, the absorption corrected (primordial) multiplicity ratio will be $\mathrm{M}_{N i}^{\bar{p}} / \mathrm{M}_{N e}^{\bar{p}} \approx$ $21 \pm 7$. Now one can estimate the $\mathrm{A}_{\text {part }}$-dependence of $\bar{p}$ production corrected for absorption: $\mathrm{M}^{\bar{p}} \propto \mathrm{A}_{\text {part }}^{\alpha}$ with $\alpha=2.9_{-0.4}^{+0.3}$. This large value of $\alpha$ is evidence for antiproton production via multiple interaction of participants.

The comparison of experimental $\bar{p}$ production cross sections to model calculations is very difficult due to the strong $\bar{p}$ absorption in the nuclear environment. Nevertheless it is worthwhile to study this observable because antiproton production at beam energies far below the threshold is extremely sensitive to medium effects. Transport models studied antiproton production in nucleus-nucleus collision by taking into account the effect of the antiproton scalar and vector selfenergy in the nuclear medium. This effect leads - similarly as for antikaons - to a strong decrease of the in-medium $\bar{p}$ effective mass with increasing nuclear density and hence to a significant enhancement of the $\bar{p}$ yield 187, 188, 189].

The comparison of experimental and calculated excitation functions (for a given reaction system) is a possibility to bypass the problem of absorption as the energy dependence of $\bar{p}$ production should not be influenced by the $\bar{p}$ annihilation. The fact, that antiprotons are produced very far below threshold by highly collective processes should result in a very steep excitation function. This is demonstrated by the dotted line in Fig. 5.7 which is the prediction of a BUU calculation. The data, however, show a much flatter slope. This trend is reproduced by a RBUU calculation which considers an in-medium mass reduction of the antiproton (dashed line in Fig. 5.7 189). 

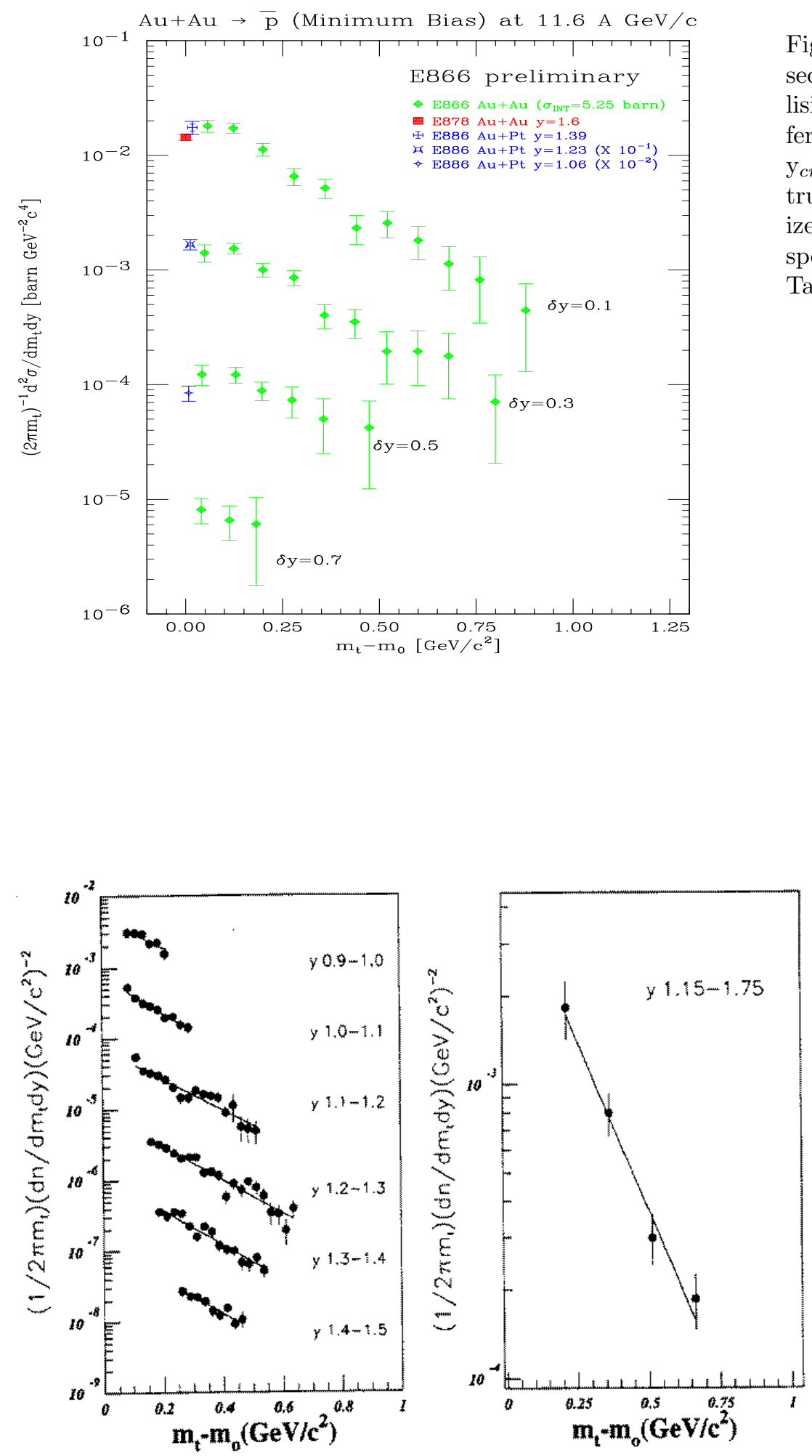

Figure 5.8: Invariant cross sections of $\bar{p}$ from $\mathrm{Au}+\mathrm{Au}$ collisions at $10.7 \mathrm{AGeV}$ in different rapidity bins $(\delta \mathrm{y}=\mid \mathrm{y}-$ $\left.\mathrm{y}_{c m} \mid\right)$. The topmost spectrum is absolutely normalized, while each successive spectrum is divided by 10 . Taken from 24].
Figure 5.9: Transverse mass spectra of $\bar{p}$ (left) and $\bar{\Lambda}$ (right) for $\mathrm{Si}+\mathrm{Au}$ central collisions at 13.7 AGeV 191. The solid lines represent exponentials fitted to the data. 
As mentioned above, the scaling of pion, kaon and antiproton production with the available energy $\mathrm{E}^{*}$ holds also at AGS energies, namely for $\mathrm{Si}+\mathrm{Al}$ at $13.7 \mathrm{AGeV}$ [136]. This finding indicates that the particles are produced according to the available phase space which just depends on beam energy (and on the mass of the collision system). The scaling is also roughly fulfilled for $\mathrm{Au}+\mathrm{Au}$ collisions at 10.7 $\mathrm{AGeV}$ [24]. However, in the heavy system the spectral slopes increase with particle mass, Fig. 5.8 shows the antiproton production cross sections measured for this system at different rapidity bins. The spectra follow an exponential with an inverse slope parameter of about $250 \mathrm{MeV}$. This value is larger than the ones for kaons and antikaons (170 - $200 \mathrm{MeV}$, see Fig. 4.21) but may be slightly smaller than the one for protons $(250-300 \mathrm{MeV})$ 24. These large inverse slope parameters indicate that the particles (including antiprotons) participate in a collective transverse expansion of the fireball.

Antiproton production has been also studied as a function of centrality in Au+Au collisions at 10.7 $\mathrm{AGeV}$ [24]. In contrast to the $\mathrm{K}^{+} / \pi^{+}$and the $\mathrm{K}^{-} / \pi^{+}$ratios which increase with increasing number of partricipants, the $\bar{p} / \pi^{+}$ratio decreases. This effect has been attributed to an enhanced absorption of $\bar{p}$ 's in central collisions [190].

In ultrarelativistic nucleus-nucleus collisions, the study of antibaryon production such as $\bar{p}$ and $\bar{\Lambda}$ may shed light on the mechanisms of antiquark production. In particular, the ratio $\bar{\Lambda} / \bar{p}$ is a measure of the the $\bar{s} / \bar{u}$ ratio which is expected to approach unity in the deconfined phase. Therefore, a $\bar{\Lambda} / \bar{p}$ ratio which is enhanced in $\mathrm{A}+\mathrm{A}$ collisions over the pp value $(\approx 0.25)$ has been considered as a signature for the transient existence of a deconfined phase. The search for such an enhancement motivates the measurement of $\bar{p}$ and $\bar{\Lambda}$ both at the AGS and the SPS. Fig. 5.9 shows the transverse mass spectra of antiprotons (left) and anti-Lambdas (right) for $\mathrm{Si}+\mathrm{Au}$ central collisions at $13.7 \mathrm{AGeV}$ [191. The inverse slope parameters of the exponential functions fitted to the data (see solid lines in Fig. 5.9) are about $180 \mathrm{MeV}$ for the antiprotons and about $190 \mathrm{MeV}$ for the anti-Lambda. The number of $\bar{\Lambda}$ is obtained by fitting the invariant mass of $\bar{p}-\pi^{+}$ pairs and correcting it for the branching ratio of $64.2 \%$ for this decay channel. The experimentally measured $\mathrm{dn} / \mathrm{dy}$ ratio of $\bar{\Lambda} / \bar{p}$ is found to be $1.02 \pm 0.21 \pm 0.10$. When correcting the $\bar{p}$ yield for the contamination due to the $\bar{\Lambda}$ decay, the $\bar{\Lambda} / \bar{p}$ ratio is $2.9 \pm 0.9 \pm 0.5$ which is higher than predicted for a system in chemical equilibrium. It rather hints at absorption effects which are more pronounced for $\bar{p}$ than for $\bar{\Lambda}$ baryons.

A transverse mass spectrum of antiprotons from $\mathrm{Pb}+\mathrm{Pb}$ at $158 \mathrm{AGeV}$ has already been shown in section 2 (Fig. 2.9). The large inverse slope parameter of $278 \pm 9$ which agrees with the one for protons $(289 \pm 7)$ was interpreted as a signature for transverse flow 44. Fig. $\overline{5.10}$ presents transverse mass spectra of $\bar{p}$ and $\bar{\Lambda}$ for $\mathrm{S}+\mathrm{S}, \mathrm{S}+\mathrm{Ag}$ and $\mathrm{S}+\mathrm{Au}$ central collisions at $200 \mathrm{AGeV}$ [192]. The solid lines correspond to exponentials fitted to the data with inverse slope parameters around $200 \mathrm{MeV}$. Similar values have been reported for antiprotons near midrapidity ( $175 \pm 6$ for central $\mathrm{S}+\mathrm{S}$ and $215 \pm$ for $\mathrm{S}+\mathrm{Pb}$ collisions at $200 \mathrm{AGeV}$ [193]). The $\bar{\Lambda} / \bar{p}$ ratio was found to be $1.9_{-0.6}^{+0.7}$ for central $\mathrm{S}+\mathrm{S}$ and $1.1_{-0.3}^{+0.4}$ for central $\mathrm{S}+\mathrm{Au}$ collisions. These values are significantly larger than the ones determined for $\mathrm{N}+\mathrm{N}(0.25 \pm 0.1), \mathrm{p}+\mathrm{S}(0.5 \pm 0.1)$ and $\mathrm{p}+\mathrm{Au}(0.3 \pm 0.1)$ collisions.

Any interpretation of the ratios of antibaryon multiplicities should take their multiplett structure as well as feeding from weak decays into account. The antinucleons are an isospin doublet. Antiproton yields, therefore, should be doubled to obtain antinucleon yields (in isospin symmetric systems). The simple hyperons consist of $\Lambda, \Sigma^{0}, \Sigma^{+}, \Sigma^{-}$. Normally only $\Lambda$ s are detected, which contain all $\Sigma^{0} \mathrm{~s}$ because of the fast electromagnetic decay of the latter. In NN interactions at SPS energies the relative abundance of $\left(\Lambda+\Sigma^{0}\right) /\left(\Lambda+\Sigma^{0}+\Sigma^{+}+\Sigma^{-}\right)$was determined to be 0.6 [60]. Thus the measured $\Lambda$ 's have to be scaled by a factor of 1.6 to obtain all hyperons. The weak decays of multistrange hyperons lead almost exculsively to $\Lambda$-hyperons. Since it is difficult to identify the $\Lambda$ 's stemming from $\Xi$-hyperon decays, the measured $\Lambda$ yield is normally an overestimate of the true yield. This effect is strongly amplified for antibaryons since the ratios $\bar{p} / \bar{\Lambda} / \Xi / \bar{\Omega}$ are much smaller than $p / \Lambda / \Xi / \Omega$ (see next section).

The significance of antibaryon yields and ratios for an assessment of the differences between $\mathrm{p}+\mathrm{p}$ and $\mathrm{A}+\mathrm{A}$ collisions is weakened by their large absorption and strangeness exchange reactions in hadronic matter. In-medium modifications of these cross sections may complicate the problem even more. A realistic treatment of these effects is a challenge for theory in general and for transport models in particular. There are two further drawbacks of the antibaryon observables: the feeding from heavier antihyperons and the in- 


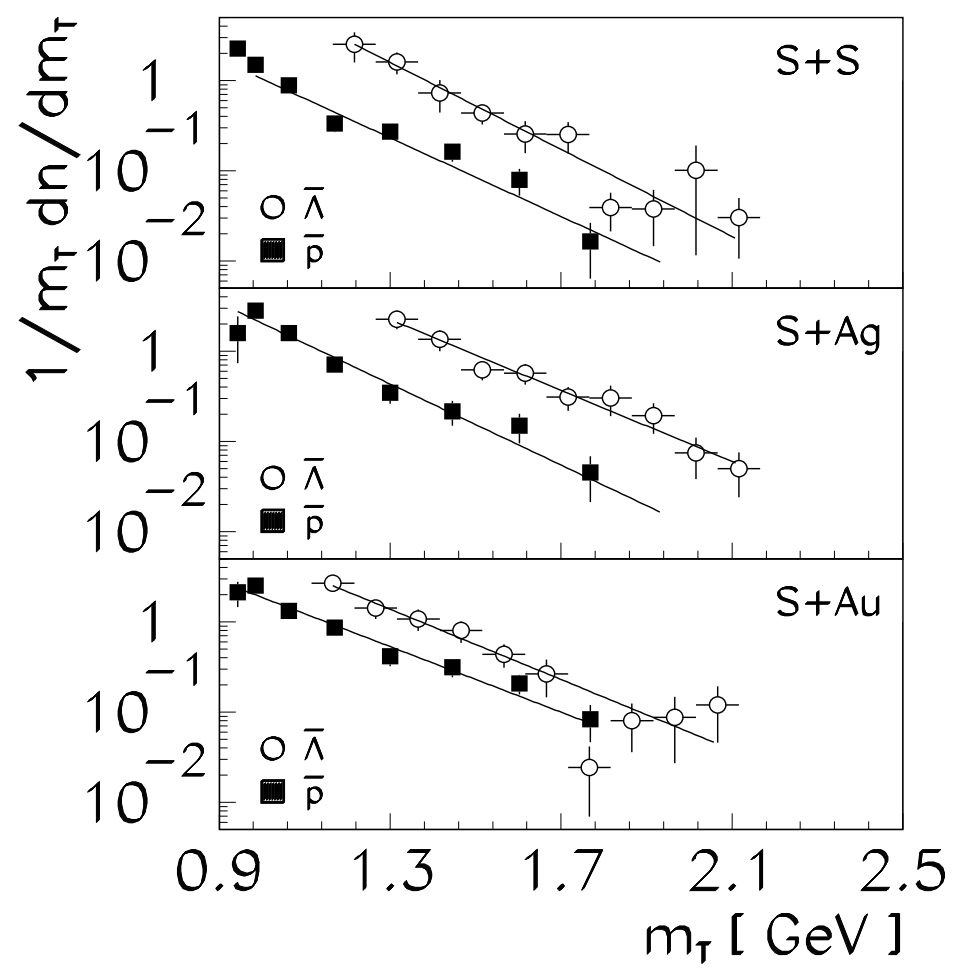

Figure 5.10: Transverse mass spectra of $\bar{p}$ and $\bar{\Lambda}$ for $\mathrm{S}+\mathrm{S}, \mathrm{S}+\mathrm{Ag}$ and $\mathrm{S}+\mathrm{Au}$ central collisions at 200 $\mathrm{AGeV}$ 192]. The solid lines correspond to exponentials fitted to the data.

complete coverage of phase space in experiments. A large fraction of the data on antibaryons was obtained from light projectiles $(\mathrm{Si}, \mathrm{S})$ on heavy targets in limited ranges of rapidities. Since baryon densities have a strong forward-backward asymmetry in these collision systems, antibaryon absorption will exhibit a similar qualitative dependence. Thus it is dangerous to compare target rapidity data $(\mathrm{Si}+\mathrm{Pb})$ with results from $\mathrm{S}+\mathrm{Au}$ forward from midrapidity. Furthermore, baryon densities in central collisions are higher at AGS than at SPS energies. Therefore it is again questionable to directly compare data on antibaryon yields and ratios for these two energy regimes. Our remark made earlier on the $\bar{\Lambda} / \bar{p}$ ratio being indicative of the early abundance of $\bar{s}$ quarks relative to $(\bar{u}+\bar{d})$ quarks holds only if the disappearance rate of antihyperons is similar to the absorption rate of antinucleons, or if chemical equilibrium governs the $\bar{\Lambda} / \bar{p}$ ratio.

Up to now there is no final answer to the question whether the strangeness enhancement observed at SPS energies (i.e. $\mathrm{K} / \pi$ or $\bar{\Lambda} / \bar{p}$ ratio) can be understood in a purely hadronic scenario as it is the case at SIS and AGS energies or whether new processes have to be invoked. Since nuclear collisions are true multibody interactions involving the strong force at low momentum transfer, a rigorous theoretical treatment of the dynamical details of heavy ion collisions is not possible. Therefore phenomenological models are used to predict the outcome of a nuclear collision. In most of these models the elementary cross sections between hadrons are used to calculate what happens to the incoming nucleons and produced particles. Such models are necessarily based on many, to some extend arbitrary, assumptions. It is beyond the scope of this survey to enter into a discussion on the ingredients of the different models. Here we only point out that they can describe all the features of meson production from SIS to AGS energies albeit with sometimes different and contradicting assumptions (for the special $\mathrm{K}^{-}$enhancement below threshold see chapter 4). At SPS energies, however, the experimental data can be reproduced by model calculations only if new collective phenomena are invoked which imply either the fusion of strings (RQMD [177], VENUS 178]) or a modification of the strange quark content in the sea quarks (DPM 194]). 
Table 5.1: Particle multiplicities and ratios as measured in central $\mathrm{Pb}+\mathrm{Pb}$ collisions at $158 \mathrm{AGeV}$ by NA49 in comparison to the results of ALCOR model calculations [197].

\begin{tabular}{|c|c|c|c|}
\hline particle type or ratio & NA49 & reference & ALCOR \\
\hline $\mathrm{h}^{-}$ & 680 & 42 & 730 \\
\hline $\mathrm{K}_{s}^{0}$ & 66 & 33. & 63 \\
\hline participant baryons & 384 & 775 & 390 \\
\hline $\bar{\Lambda} / \Lambda$ & 0.2 & 33 & 0.3 \\
\hline $\mathrm{K}^{+} / \mathrm{K}^{-}$ & 1.8 & 33 & 2.0 \\
\hline$(\mathrm{K}+\bar{K}) /<\pi>$ & 0.13 & 33 & 0.13 \\
\hline$\Xi^{-} / \Lambda$ & $0.13 \pm 0.04$ & 199 & 0.13 \\
\hline $\bar{\Omega} / \Omega$ & $0.42 \pm 0.12$ & 200 & 1.5 \\
\hline$\Omega^{-} / \Xi^{-}$ & $0.19 \pm 0.04$ & 200 & 0.18 \\
\hline $\bar{\Omega} / \bar{\Xi}_{+}$ & $0.30 \pm 0.09$ & 200 & 0.37 \\
\hline$\left(\Omega^{-}+\bar{\Omega}\right) /\left(\Xi^{-}+\bar{\Xi}_{+}\right)$ & $0.21 \pm 0.03$ & 200 & 0.25 \\
\hline
\end{tabular}

\subsection{Multistrange hyperons}

In a baryon-rich environment hyperons are formed more easily than antihyperons. Hyperons may contain more than one strange quark. The $\Xi^{0,-}$ and $\Omega^{-}$are made up of two strange ( + a light) and three strange quarks, respectively. Their production requires the creation of two and three $s \bar{s}$ pairs and is therefore strongly suppressed. To first order an $\bar{\Omega}$ is produced with the same probablilty as the $\Omega$, because both are made up of newly created quarks only. The $\bar{\Xi}$ is suppressed relativ to the $\Xi$, because an additional light antiquark has to be created in the formation of the former. In $\mathrm{A}+\mathrm{A}$ collisions one therefore expects that the production probability behaves like $\bar{\Omega} / \Omega>\Xi / \Xi>\bar{\Lambda} / \Lambda$. It has been speculated that in the relatively high $s \bar{s}$ density environment reached in a Quark-Gluon-Plasma, the multistrange hyperons will be more enhanced than the simple $\Lambda$ and $\Sigma$ particles [195, 196] since the hyperon abundance should be proportional to the density of the s-quarks raised to the power $\mathrm{n}_{s}$ with $\mathrm{n}_{s}$ being the number of strange quarks in the respective hyperon. A similar behavior is found by microscopic model calculations which introduce in addition to the purely hadronic processes chromoelectric flux tubes (colour ropes) [177 and by the transchemistry models 197] which describe the hadronization of quark matter.

Multistrange hyperons are difficult to detect because of their complicated decay topology: for example, a $\Xi^{-}$with $c \tau=4.9 \mathrm{~cm}$ decays into a $\Lambda \pi^{-}$leading to a kink in the trajectory of a negatively charged particle. The $\Lambda$ with $c \tau=7.9 \mathrm{~cm}$ leaves no visible track but creates the characteristic $\mathrm{V}^{0}$ toplogy when it decays with the $\mathrm{V}^{0}$ pointing to the kink. The $\Omega^{-}$has the same decay topology with the $\pi^{-}$replaced by a $\mathrm{K}^{-}$ meson. In high energy nuclear collisions these topologies are burried in the high multiplicities of the charged particle tracks. Experimental data on multistrange hyperons were published on $\mathrm{p}+{ }^{32} \mathrm{~S}$ and ${ }^{32} \mathrm{~S}+{ }^{32} \mathrm{~S}[31]$ for transverse momenta above $1 \mathrm{GeV} / \mathrm{c}$. Preliminary data on $\Xi$ production in ${ }^{32} \mathrm{~S}+{ }^{32} \mathrm{~S}$ collisions from NA35 at lower $\mathrm{p}_{T}$ have recently been shown 198 . New preliminary results from $\mathrm{Pb}+\mathrm{Pb}$ collisions obtained by WA97 and NA49 also become available [200, 199] Some relevant particle ratios including multistrange hyperons observed in $\mathrm{Pb}+\mathrm{Pb}$ collisions are summarized in Fig. 5.11] [31]. The ratios of multistrange to normal hyperons increase with system size and are larger for antihyperons than for hyperons. In Table 5.1, the experimental numbers for $\mathrm{Pb}+\mathrm{Pb}$ collisions are compared to the predictions of the ALCOR model 197.

Particle ratios including multistrange antihyperons are affected by similar problems as those including "normal" antihyperons and antinucleons. The large strangeness exchange cross sections will influence strongly the abundance of antihyperons and it will be difficult or even impossible to calculate the different contributions to the particle yields in a nonequilibrium situation. Only if the particle abundances come close to chemical equilibrium values one can hope to predict them quantitatively. Microscopic model studies may help to verify whether such conditions are reached in central A+A collisions. 


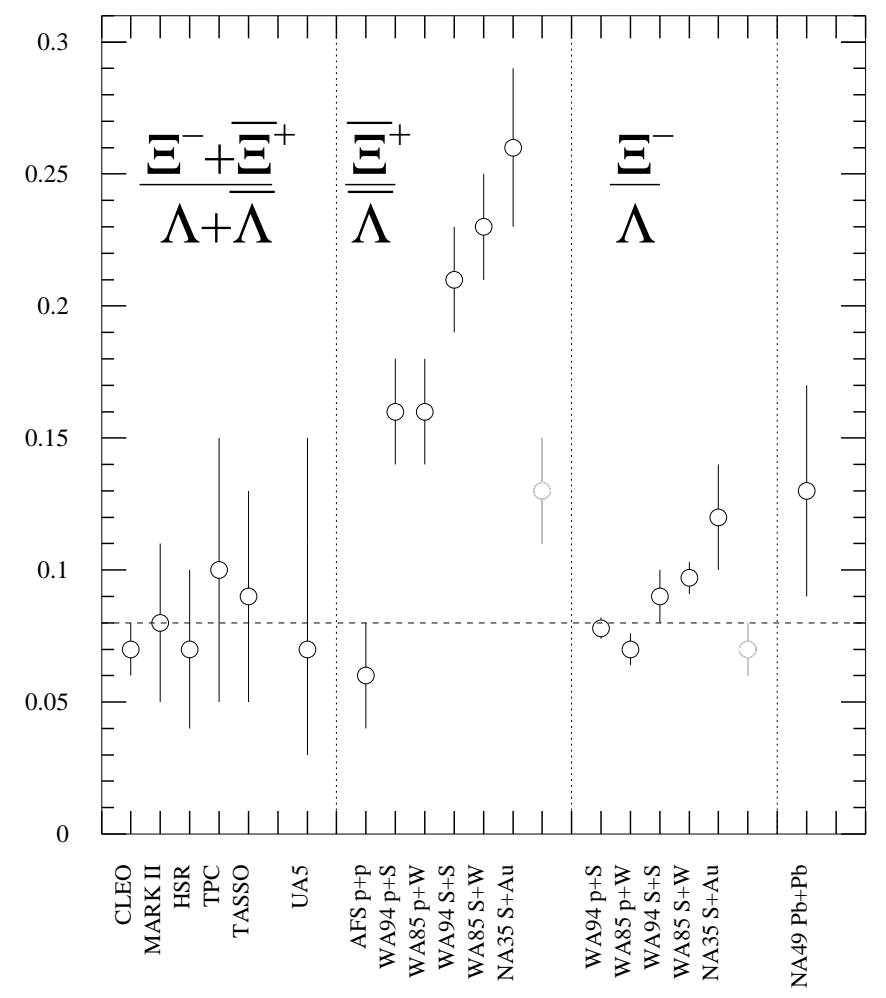

Figure 5.11: Comparison of $\Xi^{-} / \Lambda$ and $\bar{\Xi}^{+} / \bar{\Lambda}$ ratios obtained in different $\mathrm{p}+\mathrm{p}, \mathrm{p}+\bar{p}, \mathrm{e}^{+}+\mathrm{e}^{-}$and $\mathrm{A}+\mathrm{A}$ collisions at CERN SPS energies (taken from [199]). 


\section{Chapter 6}

\section{Conclusions}

Hadronic particle production is an important and at high energies the dominant process in nucleus-nucleus collisions. Therefore its study and understanding is essential for the physics of highly compressed and heated hadronic matter. The fundamental theory for the description of hadronic particle production is Quantum Chromodynamics. However, within the framework of this theory soft processes cannot be calculated, neither for nucleon-nucleon nor nucleus-nucleus collisions. Therefore, phenomenological models are invoked to describe and interprete the experimental results. These models are especially helpful to identify and understand differences in the particle production characteristics between $\mathrm{N}+\mathrm{N}$ and $\mathrm{A}+\mathrm{A}$ collisions. The combination of phenomenology and experiment in the analysis of nuclear collision data offer the possibility to investigate fundamental questions in strong interaction physics like the nuclear equation of state (EOS), in-medium modifications of hadron properties and the deconfinement phase transition. Other interesting topics concern thermal and chemical equilibrium as well as energy and baryon densities reached in nuclear collisions.

The measured particle yields are in accordance with the assumption of a fireball being close to chemical equilibrium, both for $\mathrm{A}+\mathrm{A}$ and $\mathrm{p}+\mathrm{p}$ collisions with the exception that in the $\mathrm{p}+\mathrm{p}$ system strange particles are suppressed. The widths of rapidity distributions agree with those from a thermal source at low beam (SIS) energies but are much larger at high (CERN SPS) energies, again both for $\mathrm{A}+\mathrm{A}$ and $\mathrm{p}+\mathrm{p}$ reactions. These observations at high beam energies can be explained by the formation of strings and their fragmentation into hadrons which are equally distributed in phase space. This process seems to be a common feature to both $\mathrm{p}+\mathrm{p}$ and $\mathrm{A}+\mathrm{A}$ collisions. In central collisions of heavy nuclei and at all energies, the transverse momentum distributions of the produced particles show evidence for a transverse expansion of the fireball.

The pion phase-space distributions suggest that the bulk of the pions freeze out in a late and dilute stage of the thermal fireball. At low (SIS) energies, however, an azimuthally anisotropic emission pattern (varying with pion energy) was found which is explained by rescattering of pions from the spectators. This observation indicates that high-energy pions stem from an early, dense and hot phase of the reaction.

The enhanced production of strange particles has been observed at all beam energies. At low (SIS) and intermediate (AGS) beam energies, the kaon to pion ratio increases with the number of participating nucleons. This experimental finding is a signature for the important role of collective effects: the energy needed to create a kaon is accumulated in sequential processes involving more than two nucleons. On the other hand, at high (SPS) energies the kaon to pion ratios stay remarkably constant in the range from 50 to 300 participating nucleons.

At beam energies below 1.6 AGeV, collisions between high-energy pions (or $\Delta$ resonances) and nucleons are considered to be the dominant process of "subthreshold" $\mathrm{K}^{+}$production. This process depends sensitively on the baryon density and on the thermal energy of the fireball and hence on the compressibility of nuclear matter. The determination of the EOS far from normal nuclear density is an old challenge in nuclear physics and is of great importance also for astrophysics. Heavy ion collisions at beam energies of 1-2 AGeV create a fireball with baryon densities of 2-3 $\rho_{0}$ at moderate temperatures and thus are well suited to study the properties of nuclear matter far from its ground state. The analysis of available kaon production 
data by transport calculations favors a soft EOS. Systematic experimental studies of $\mathrm{K}^{+}$production for light and heavy collision systems and at different beam energies are needed to constrain considerably the range of possible values for the nuclear compressibility.

According to calculations based on effective chiral Lagrangians the in-medium mass of the antikaon is predicted to drop to about half its vacuum value at baryon densities of $2 \rho_{0}$. Experimental evidence for an enhanced $\mathrm{K}^{-}$production yield in nucleus-nucleus collisions confirms the underlying concept. Furthermore, the in-medium potentials of kaons and antikaons are expected to influence considerably their azimuthal emission pattern. Absence of directed in-plane flow of $\mathrm{K}^{+}$mesons and an enhanced $\mathrm{K}^{+}$out-of-plane emission have been explained by a repulsive $\mathrm{KN}$ in-medium potential. A crucial test of the concept of in-medium KN potentials will be the azimuthal distribution of antikaons which will be measured in future experiments with KaoS and FOPI at SIS/GSI.

From SIS to SPS energies a dramatic change of the hadrochemical composition of the created fireball takes place: a transition from the baryonic to the pionic regime. The pion/baryon ratio increases from about $0.1-0.2$ at beam energies of 1-2 AGeV to about 1 at $10 \mathrm{AGeV}$ and up to 5 at 100-200 AGeV. The strangeness/baryon ratio increases from 0.001 at $2 \mathrm{AGeV}$ to 0.07 at $10 \mathrm{AGeV}$ and up to 0.4 at $200 \mathrm{AGeV}$. The experiments performed so far at AGS and SPS have shown that the nuclei are still nearly stopped creating energy densities as high as ten times the nuclear ground state density. Such an energy density is one of the necessary conditions for the creation of the Quark-Gluon-Plasma. Some of the hadronic signals, which were predicted to indicate its transient existence, have also been seen. Measurements of the J/ $\Psi$ suppression as seen in $\mu^{+} \mu^{-}$pairs also ask for new nonhadronic phenomena. The question, whether the QGP is already seen at SPS or even AGS energies has so far no commonly accepted answer. The study of the phase transition from a state of deconfined quarks and gluons back to the hadronic world will be the major aim of new experiments at new accelerators at BNL (STAR, PHENIX etc. at RHIC) and CERN (ALICE at LHC). 


\section{Bibliography}

[1] R.Stock, Phys.Rep. 135 (1986) 259

[2] H.Stöcker, W.Greiner, W.Scheid, Z.Phys. A 286 (1978) 121

[3] K.A.Gustafsson and the Plastic Ball Collaboration, Phys.Rev.Lett. 52 (1984) 1990

[4] R.Renford and the Streamer Chamber Collaboration, Phys.Rev.Lett. 53 (1984) 763

[5] W.Greiner and H.Stöcker (Editors), "The Nuclear Equation of State", Plenum Press, Series B: Physics Vol. 216 A

[6] W.Reisdorf and H.G.Ritter, An.Rev.Nucl.Part.Sci. 47 (1997) 663

[7] J.Bartke, Int. Journ. Mod. Phys. A4 (1989) 1319

[8] J.Stachel and G.R.Young, An. Rev. Nucl. Part. Sci. 42 (1992) 537

[9] J.B.Cugnon et al., Nucl.Phys. A 360 (1981) 444

[10] J.Hüfner and J.Knoll, Nucl.Phys. A 290 (1977) 460

[11] P.Danielewicz and G.Odyniec, Phys.Lett. 157 B (1985) 147

[12] U.A.Wiedemann, P.Scotto and U.Heinz, Phys.Rev. C53 (1996) 918

[13] R.Averbeck, Proceedings of the IV. TAPS Workshop, Mont Sainte Odile, France, Sept. 1997

[14] P.Braun-Munzinger et al., Phys.Lett. B344 (1995) 43

[15] C. Spieles, H. Stöcker and C. Greiner, The Eur. Phys. J. C2(1998)351

[16] P.Braun-Munzinger et al., Phys.Lett. B356 (1996) 1

[17] B.Hong et al., Phys.Lett. B 407 (1997) 115 and Phys.Rev. C 57 (1998) 244

[18] R.Stock, Nucl. Phys. A 630 (1998)

[19] F.Becattini, Z.Phys. C76 (1996) 485

[20] D.Pelte et al., Z.Phys. A 357 (1997) 215

[21] D.Best et al., Nucl. Phys. A 625 (1997) 307

[22] D.Pelte et al., Z.Phys. A 359 (1997) 55

[23] J.Stachel, Nucl.Phys. A610 (1996) 509c

[24] L.Ahle and the E802 Collaboration, Nucl.Phys. A 610 (1996) 139c

[25] J.Barrette et al., Phys.Lett. B 351 (1995) 93

[26] R.Lacasse for the E877 Collaboration Nucl.Phys. A610 (1996) 153c 
[27] S.Ahmad et al., Phys.Lett. B 382 (1996) 35

[28] T.Abbott et al., Phys.Rev. C 50 (1994) 1024

[29] V. Blobel et al., Nucl.Phys. B 69 (74) 454

[30] J.Guenther, PhD Thesis, University of Frankfurt 1997

[31] P.Seyboth, J.Phys. G23 (1997) 1787c

[32] S.A. Bass et al., Progr. Part. Nucl. Phys. 41 in print, and nucl-th/9803035

[33] C. Bormann and the NA49 Collaboration, J.Phys. G 23 (1997) 1817c

[34] J. Bächler et al.,Z. Phys. C58 (1993) 367

[35] T. Alber et al., Z. Phys. C64 (1994) 195

[36] Y. Eisenberg et al., Nucl.Phys. B 154 (79) 239

[37] K. Jaeger et al., Phys. Rev. D11 (1975) 2405

[38] J.D. Bjorken, Phys. Rev. D27 (1983) 140

[39] R. Hagedorn Suppl. Nuov. Cimento 3 (1965) 147 and Riv. Nuov. Cimento 6 (1983) 1

[40] J. Bächler et al., Phys.Rev.Lett. 72 (94) 1419

[41] T.Alber et al., Eur. Phys. J. C 2 (1998) 643

[42] P.G.Jones and the NA49 Collaboration, Nucl.Phys. A610 (1996) 188c

[43] A. M. Rossi et al., Nucl.Phys. B84 (75) 269

[44] I.G.Bearden et al., Phys.Rev.Lett. 78 (1997) 2080

[45] A.Leonidov, M. Nardi, H. Satz, Z. Phys. C74 (1997) 535 and S. Jeon, J. Kapusta, Phys. Rev C56(1997) 468

[46] E.Schnedermann, J.Sollfrank and U.Heinz, Phys.Rev. C48 (1993) 2462

[47] M.A.Lisa et al., Phys.Rev.Lett. 75 (1995) 2662

[48] S.C.Jeong et al., Phys.Rev.Lett. 72 (1994) 3468

[49] P.J.Siemens and J.O.Rasmussen, Phys.Rev.Lett. 40 (1979) 880

[50] N.Herrmann, Nucl.Phys. A610 (1996) 49c

[51] D.Miskowiec et al., Phys.Rev.Lett. 72 (1994) 3650

[52] W.Ahner et al., Phys.Lett. B 393 (1997) 31

[53] R.Averbeck et al., Z.Phys. A 359 (1997) 65

[54] A.Shor et al., Phys.Rev.Lett. 63 (1989) 2192

[55] A.Schröter et al., Z.Phys. A 350 (1994) 101

[56] A. Abdivaliev et al., JINR, P1-82-507 and GSI-tr-86-01

[57] P. Jacobs and the NA49 Collaboration, Proc. of the 3rd Intern. Conf. on the Physics and Astrophysics of the Quark Gluon Plasma, Jaipur, India, 17-21 March 1997

[58] H.Ströbele, Nucl. Phys. A 610 (1996) 102c 
[59] M. Gazdzicki and D. Röhrich, Z. Phys. C71 (96) 55

[60] A.K. Wroblewski, Acta Phys. Polon. B16 (1985) 379

[61] B.J.VerWest and R.A.Arndt, Phys.Rev. C 25 (1982) 1979

[62] S.Huber und J.Aichelin, Nucl.Phys. A587 (1994)

[63] S.A.Bass et al., Phys.Rev. C 51 (1995) 3343

[64] S.Teis et al., Z.Phys. A 356 (1997) 421

[65] I.Navon et al., Phys.Rev.Lett. 42 (1979) 1465

[66] K.Nakai et al., Phys.Rev.Lett. 44 (1980) 1446

[67] H.W. Barz et al., nucl-th/9711064

[68] J.W.Harris et al., Phys.Lett. 153 B (1985) 377

[69] J.W.Harris et al., Phys.Rev.Lett. 58 (1987) 463

[70] F.D.Berg et al., Phys.Rev.Lett. 72 (1994) 977

[71] A.Wagner et al., Phys.Lett. B 420(1998) 20

[72] O.Schwalb et al., Phys.Lett.B321 (1994) 20 and V.Metag private communication: the pion cross sections from $\mathrm{Au}+\mathrm{Au}$ at $1 \mathrm{AGeV}$ have been renormalized by 0.6 due to a recent analysis

[73] A.Wagner, Thesis Th Darmstadt 1996 and to be published

[74] M. Gazdzicki and D. Röhrich, Z. Phys. C65 (95) 215

[75] H. Appelshäuser et al., Eur. Phys. J. C 2, (1998) 661

[76] R.Brockmann et al., Phys.Rev.Lett. 53 (1984) 2012

[77] Ch.Müntz et al., Z.Phys. A 357 (1997) 399

[78] E.L.Hjort et al., Phys.Rev.Lett. 79 (1997) 4345

[79] S.Nagamiya et al., Phys.Rev. C 24 (1981) 971

[80] S.A.Bass et al., Phys.Rev. C 50 (1994) 2167

[81] W.Weinhold, Acta Physica Polonica B 27 (1996) 3249 and and submitted to Phys.Lett. B (nuclth/9710014)

[82] Ch.Müntz et al., Z.Phys.A 352 (1995) 175

[83] A.Foerster, diploma thesis, TU Darmstadt, 1998, unpublished

[84] D. Röhrich for the NA35 Collaboration, Nucl. Phys. A566 (1994) 35c

[85] K.L.Wolf et al., Phys.Rev.Lett. 42 (1979) 1448 and Phys.Rev. C26 (1982) 2572

[86] H.H.Gutbrod et al., Phys.Lett. 216B (1989) 267

[87] M.Demoulins et al., Phys.Lett. B 241 (1990) 476

[88] J.Gosset et al., Phys.Lett. B 247 (1990) 233

[89] H.H.Gutbrod et al., Phys.Rev. C42 (1990) 640

[90] Y.Leifels et al., Phys.Rev.Lett. 71 (1993) 963 
[91] D.Lambrecht et al., Z.Phys. A 350 (1994) 115

[92] D.Brill et al., Z.Phys. A 355 (1996) 61

[93] D.Keane et al., Proceedings of the 4th Nuclear Dynamics Workshop, Copper Mountains, Colorado 1986 (unpublished)

[94] P.Danielewicz et al., Phys.Rev. C38 (1988) 120

[95] J.Gosset et al. Phys.Rev.Lett. 62 (1989) 1251

[96] J.C.Kintner et al., Phys.Rev.Lett. 78 (1997) 4165

[97] Bao-An Li, Nucl.Phys. A570 (1994) 797

[98] Bao-An Li and C.M.Ko, Phys.Rev. C 53 (1996) R22

[99] C.Hartnack, private communication

[100] D.Brill et al., Phys.Rev.Lett 71 (1993) 336

[101] L.B.Venema et al., Phys.Rev.Lett 71 (1993) 835

[102] D.Brill et al., Z.Phys. A 357 (1997) 207

[103] S.A.Bass et al., Phys.Rev.Lett 71 (1993) 1144

[104] J.Barrette et al., Phys.Rev. C 55 (1997) 1420

[105] H.Appelshäuser et al., Phys.Rev.Lett. 80 (1998) 4136

[106] Nu Xu for the NA44 Collaboration, Nucl.Phys. A610 (1996) 175c

[107] S.Teis et al., Z.Phys. A 359 (1997) 297

[108] C.B.Dover and G.E.Walker, Phys.Rep.89 (1982) 1

[109] M.Cieslak, PhD-Thesis, Jagiell. Univ. Cracow, 1996

[110] M.Mang, PhD-Thesis, Univ. Frankfurt, 1997

[111] R.Barth et al., Phys.Rev.Lett. 78 (1997) 4007

[112] J.Randrup and C.M.Ko, Nucl.Phys. A 343 (1980) 519 and Nucl.Phys. 411 (1983) 537

[113] J.Randrup, Phys.Lett. 99B (1981) 9

[114] C.M.Ko, Phys.Rev. C 23 (1981) 2760

[115] J.Cugnon and R.M.Lombard, Nucl.Phys.A 422 (1984) 635

[116] T.Maruyama et al., Nucl.Phys. A 573 (1994) 653

[117] J.Aichelin and C.M Ko, Phys.Rev.Lett. 55 (1985) 2661

[118] C.Fuchs et al., Phys.Rev. C 56 (1997) R606

[119] G.Q.Li et al., Phys.Lett. B 349 (1995) 405

[120] D.B.Kaplan and A.E.Nelson, Phys.Lett. B 175 (1986) 57

[121] G.E.Brown et al., Phys.Rev. C 43 (1991) 1881

[122] S.Klimt et al., Phys.Lett. B 249 (1990) 386

[123] X.S.Fang et al., Nucl.Phys. A 575 (1994) 766 
[124] W.Cassing et al., Nucl.Phys. A614 (1997) 415

[125] E.Bratkovskaya et al., Nucl.Phys. A622 (1997) 593

[126] G.Q.Li et al.,Phys.Rev.Lett. 74 (1995) 235

[127] G.Q.Li, C.M.Ko and G.E.Brown, Phys.Lett. B 381 (1996) 17 and G.Q.Li, private communication

[128] J.Ritman et al., Z.Phys. A 352 (1995) 355

[129] S.Schnetzer et al., Phys.Rev.Lett. 49 (1982) 989 and Phys.Rev. C 40 (1989) 640

[130] J.W.Harris et al., Phys.Rev.Lett. 47 (1981) 229

[131] M.Anikina et al., Z.Phys. C 25 (1984) 1 and Phys.Rev.Lett. 50 (1983) 1971

[132] V.Metag, Proc. of the Nucleus-Nucleus 1997, Gatlinburg, USA, GSI-Preprint-97-43, 1997

[133] C.Sturm, PhD Thesis TU Darmstadt 1998

[134] F.Laue, Dissertation Univ. Frankfurt 1997 and to be published

[135] C.Hartnack et al. Nucl.Phys. A 580 (1994) 643

[136] A.Shor et al., Phys.Lett. B274 (1992) 11

[137] A.Gillitzer et al.,Proc. XXXIII Int. Winter Meeting on Nuclear Physics, Bormio (Italy), Jan 23-27, $1995,(1995) 496$

[138] P.Kienle and A.Gillitzer, Proc. of the int. Conf. Structure of Vacuum \& Elementary Matter, Wilderness, South Africa, March 10-16, 1996. World Scientific 1997

[139] W.Zwermann and B.Schürmann, Phys.Lett. B 145 (1984) 315

[140] V.S.Pantuev, S.N.Filippov and A.B.Kurepin, Nucl.Phys. A585 (1995) 13c

[141] S.Nagamiya, Phys.Rev.Lett. 49 (1982) 1383

[142] P.Senger et al., Nucl. Instr. Meth. A 327 (1993) 393

[143] R.Elmér et al., Phys.Rev.Lett. 77 (1996) 4884

[144] Z.S.Wang et al., Phys.Rev.Lett. 79 (1997) 4096.

[145] Y.Shin et al., nucl-ex/9807003 and Phys.Rev.Lett. (1998) in print

[146] W.Schürmann and W.Zwermann, Phys. Lett. B 183 (1987) 31

[147] A.Sibirtsev, Phys.Lett. B 359 (1995) 29

[148] H.Müller, Z.Phys. A 353 (1995) 103

[149] J.Balewski et al., Phys.Lett. B 388 (1996) 859 and Phys.Lett. B in print

[150] G.Agakichiev et al., Phys.Rev.Lett 75 (1995) 1272

[151] J.Porter et al., Phys.Rev.Lett. 79 (1997) 1229

[152] T.Waas et al., Phys.Lett. B 379 (1996) 34

[153] J.Schaffner and I.Mishustin, Phys.Rev. C 53 (1996) 1416

[154] M.Lutz, Phys.Lett. B 426 (1998) 12

[155] E.Friedman, A.Gal and C.J.Batty, Phys.Lett. B 308 (1993) 6 
[156] E.Friedman et al., Phys.Rev. C55 (1997) 1304

[157] J.Schaffner-Bielich, J.Bondorf, I.Mishustin, Nucl. Phys. A 625 (1997) 325

[158] G.E.Brown et al., Nucl.Phys. A567 (1994) 937

[159] R.Knorren, M.Prakash, P.J.Ellis, Phys.Rev. C 52 (1995) 3470

[160] H.Fujii, T.Maruyama, T.Tatsumi, Nucl.Phys. A597 (1996) 645

[161] G.E.Brown and H.A.Bethe, Astrophys.Jour. 423 (1994) 659

[162] G.Q.Li, C.H.Lee and G.E.Brown, Phys.Rev.Lett. 79 (1997) 5214

[163] G.Q.Li, C.H.Lee and G.E.Brown, Nuc.Phys. A 625 (1997) 372

[164] G.Q.Li et al., Phys.Rev. C 54 (1996) R2159

[165] G.Q.Li et al., Phys.Lett. B 329 (1994) 149

[166] CERN HERA Report 84-01 (1984)

[167] W.J.Hogan et al., Phys.Rev. 166 (1968) 1472

[168] A.Sibirtsev, W.Cassing, C.M.Ko, Z.Phys. A387 (1997) 101

[169] S.V.Efremov and E.Y.Par'ev, Z.Phys. A 351 (1995) 447

[170] S.V.Efremov and E.Y.Par'ev, Phys. of Atomic Nuclei 57 (1994) 532

[171] K.Tsushima, S.W.Huang and A.Faessler, J.Phys. G 21 (1995) 33

[172] C.M.Ko, Phys.Rev. C 29 (1984) 2169

[173] E.Bratkovskaya and W.Cassing, private communication

[174] C.A.Ogilvie, J.Phys. G23 (1997) 1803c and submitted to Phys. Rev. C

[175] H. Sorge et al., Phys. Lett. B289 (1992) 6

[176] M. Hofmann et al., Nucl. Phys. A566(1994)15c

[177] H.Sorge, Phys.Rev. C52 (1995) 3291

[178] K.Werner and J.Aichelin, Phys.Lett. B 308 (1993) 372

[179] M.C. Abreu et al., Phys.Lett. B 368 (1996) 239

[180] V.Friese et al., J.Phys. G 23 (1997) 1837c

[181] R.Novotny, IEEE Trans. on Nucl. Sc. 38 (1991) 379

[182] W.Ehehalt and U.Mosel, private communication

[183] R.Albrecht et al., Phys.Lett. B 361 (1995) 1

[184] A.A.Baldin et al., JETP Lett. 47 (1988) 137

[185] P.Koch and C.B.Dover, Phys.Rev. C 40 (1989) 145

[186] Landoldt-Börnstein, New Series, Group I, Vol.12, Springer Verlag 1988, Germany

[187] G.Q.Li et al., Phys.Rev. C 49 (1994) 1139

[188] G.Batko et al., J.Phys. G: Nucl.Part. Phys. 20 (1994) 461 
[189] S.Teis et al., Phys.Lett. B 319 (1993) 47 and Phys.Rev. C 50 (1994) 388

[190] H.Sako et al., Proc. of HIPAGS'96, WSU-NP-96-16 (1996) 67

[191] Y.Wu and the E802/E859 Collaboration, Proc. of HIPAGS'96, WSU-NP-96-16 (1996) 37

[192] T.Alber et al.,Phys.Lett.B 366 (1996) 56

[193] J.Dodd et al., Quark Matter 1995, Monterey, California, USA

[194] A.Capella et al., Z.Phys. C 70 (1996) 507

[195] J.Rafelski and B. Müller, Phys. Rev. Lett. 48 (1982) 1066

[196] V.Koch, B. Müller, J. Rafelski, Phys. Rep. 142 (1986) 391

[197] J.Zimányi et al., APH NS Heavy Ion Physics Vol.4 (1996) 15

[198] W.Retyk, NA35 Collaboration, J.Phys. G23 (1997) 1845c

[199] G.Odyniec, NA49 Collaboration, J.Phys. G23 (1997) 1827c

[200] A.K.Holme, WA97 Collaboration, J.Phys. G23 (1997) 1851c 